\title{
Short-term variability of the Sun-Earth system: an overview of progress made during the CAWSES-II period
}

\author{
Nat Gopalswamy ${ }^{1 *}$, Bruce Tsurutani ${ }^{2}$ and Yihua Yan $^{3}$
}

\begin{abstract}
This paper presents an overview of results obtained during the CAWSES-II period on the short-term variability of the Sun and how it affects the near-Earth space environment. CAWSES-II was planned to examine the behavior of the solar-terrestrial system as the solar activity climbed to its maximum phase in solar cycle 24. After a deep minimum following cycle 23, the Sun climbed to a very weak maximum in terms of the sunspot number in cycle 24 (MiniMax24), so many of the results presented here refer to this weak activity in comparison with cycle 23. The short-term variability that has immediate consequence to Earth and geospace manifests as solar eruptions from closed-field regions and high-speed streams from coronal holes. Both electromagnetic (flares) and mass emissions (coronal mass ejections - CMEs) are involved in solar eruptions, while coronal holes result in high-speed streams that collide with slow wind forming the so-called corotating interaction regions (CIRs). Fast CMEs affect Earth via leading shocks accelerating energetic particles and creating large geomagnetic storms. CIRs and their trailing high-speed streams (HSSs), on the other hand, are responsible for recurrent small geomagnetic storms and extended days of auroral zone activity, respectively. The latter leads to the acceleration of relativistic magnetospheric 'killer' electrons. One of the major consequences of the weak solar activity is the altered physical state of the heliosphere that has serious implications for the shock-driving and storm-causing properties of CMEs. Finally, a discussion is presented on extreme space weather events prompted by the 23 July 2012 super storm event that occurred on the backside of the Sun. Many of these studies were enabled by the simultaneous availability of remote sensing and in situ observations from multiple vantage points with respect to the Sun-Earth line.
\end{abstract}

Keywords: Solar activity; Space weather; Coronal mass ejections; Flares; Solar energetic particle events; Geospace impact; Geomagnetic storms

\section{Review} Introduction

The second phase of the Climate and Weather of the Sun-Earth System (CAWSES-II) was organized into task groups (TGs). Task Group 3 (TG3) was focused on the short-term variability of the Sun-Earth system. Solar variability on time scales up to 11 years was relevant to TG3. The relevant variability occurs in the mass and electromagnetic outputs of the Sun. The mass output has three forms: the solar wind, coronal mass ejections (CMEs), and solar energetic particles (SEPs). The electromagnetic

\footnotetext{
*Correspondence: nat.gopalswamy@nasa.gov

'Solar Physics Laboratory, Code 671, Heliophysics Division, NASA Goddard Space Flight Center, Greenbelt, MD 20771, USA

Full list of author information is available at the end of the article
}

output consists of the quasi-steady black body radiation with the superposition of flare emission. The mass and electromagnetic emissions are often coupled: flares and CMEs represent two different manifestations of the energy release from solar source regions (e.g., Asai et al. 2013). SEPs are accelerated in fast CME-driven shocks as well as by flare reconnection (see e.g., Reames 1999, 2013). The source regions of flares and CMEs are closed magnetic field regions such as active regions and filaments (see e.g., Srivastava et al. 2014). Active regions consist of sunspots of opposite polarity at the photospheric level. Filament regions do not have sunspots but consist of opposite polarity magnetic patches. The main difference between the two regions is the magnetic field strength: hundreds of gauss in the sunspot regions vs. tens or less gauss in filament 
regions. Sunspots also contribute to the variability in total solar irradiance (TSI). While sunspots decrease the TSI, the plages that surround the sunspots increase it, resulting in higher TSI when the Sun is more active.

The solar wind has fast and slow components. The fast component is of particular interest because it can compress the upstream slow solar wind forming a corotating interaction region (CIR). Coronal holes, the source of the fast solar wind, also exhibit remarkable variability in terms of their location on the Sun and size. Perhaps even more important than the CIR is the high-speed stream proper. It carries large nonlinear Alfvén waves, whose southward components cause reconnection at the magnetopause resulting in continuous sporadic plasmasheet injections into the nightside magnetosphere. These injections of anisotropic approximately $10-$ to $100-\mathrm{keV}$ electrons cause the growth of an electromagnetic wave called 'chorus' and the chorus interacts with approximately $100-\mathrm{keV}$ electrons accelerating them to $\mathrm{MeV}$ energies (Tsurutani et al. 2006, 2010; Thorne et al. 2013).

CMEs are launched into the solar wind, so the two mass outputs interact and exchange momentum affecting the propagation characteristics of CMEs in the interplanetary medium. CMEs also interact with the upstream heliospheric current sheet and other material left over from other injections. The variability manifested as solar flares, CMEs, SEPs, and high-speed solar wind streams directly affects space weather on short time scales. As noted above, all these phenomena are coupled not only near the Sun but throughout the inner heliosphere, including geospace and Earth's ionosphere and atmosphere where the impact can be felt (Verkhoglyadova et al. 2014; Mannucci et al. 2014; Tsurutani et al. 2014).

\section{Methods}

This review covers the second phase of the CAWSES program, known as CAWSES-II, which began in 2009 and ended in 2013, roughly covering the rise to the maximum phase of solar cycle 24. There is strong evidence showing that solar cycle 24 is a relatively weak cycle (Tan 2011; Basu 2013). The birth of solar cycle 24 was remarkable in that the Sun emerged from an extremely deep minimum. The maximum phase of cycle 24 is of particular interest because the sunspot number was rather small (roughly half of the cycle 23 peak). The weak solar cycle resulted in a milder space weather, but there were other complications such as longer living space debris due to the reduced atmospheric drag. SCOSTEP conducted a year-long campaign known as 'MiniMax24' to document solar events and their geospace impact during the mild maximum phase of cycle 24. Additionally, the solar mid-term and long-term quasi-periodic cycles and their possible relationships with planetary motions from longterm observations of the relative sunspot number and microwave emission at frequency of $2.80 \mathrm{GHz}$ were also investigated, and it was suggested that the mid-term solar cycles (periods $<12$ years) are closely related to the motions of the inner planets and of Jupiter (Tan and Cheng 2013).

This paper highlights some key results obtained on the variable phenomena in the Sun-Earth system during the CAWSES-II time frame. Detailed treatment of topics relevant to CAWSES-II can be found in the Living Reviews in Solar Physics: Hathaway (2010), Charbonneau (2010), Petrovay (2010), Chen (2011), Shibata and Magara (2011), Aschwanden (2011), Webb and Howard (2012), Usoskin (2013), Potgieter (2013), Lockwood (2013), Owens and Forsyth (2013).

\section{The weak solar cycle 24 and its consequences}

CAWSES-II focused on solar variability as the Sun approached the maximum of cycle 24. The rise phase of cycle 24 was already interesting because the Sun emerged from a deep solar minimum that gained particular interest among solar-terrestrial scientists (Selhorst et al. 2011; Tsurutani et al. 2011a; Dasso et al. 2012; Gopalswamy et al. 2012a; Solomon et al. 2013; Lean et al. 2014; Potgieter et al. 2014). Solar activity is typically represented by the international sunspot number (SSN), but there are many other measures, which are needed for a complete understanding of the solar variability. In particular, measurements of the polar field strength, tilt angle of the heliospheric current sheet (Smith et al. 1978), latitudes of filament locations, and coronal streamers all provide complementary information on the solar activity as the Sun climbed toward its weak maximum around 2013.

\section{Solar polar field strength}

Figure 1 shows the equatorial (sunspot number - SSN, active region microwave brightness temperature - $\mathrm{Tb}$ ) and polar (magnetic field strength) manifestations of solar cycle 24, in comparison with cycle 23 . The sunspot number (SSN) and the equatorial microwave $\mathrm{Tb}$ indicate the strength of the activity cycle. Clearly, the decline in SSN from cycles 23 to 24 is very pronounced. The Tb decline is also evident. The Tb from the northern and southern active region belt shows marked asymmetry around the solar maxima. The northern AR emission shows only a single peak during the first SSN peak, while the southern AR Tb shows double peaks, the first peak being weaker than the second peak in the two cycles. The combination of northern and southern peaks in SSN gives rise to the well-known Gnevishev gap. The Gnevishev gap was somewhat wider during the cycle 24 maximum. The polar field strength during the prolonged cycle $23 / 24$ minimum was considerably smaller than that during the cycle 22/23 minimum. During solar 

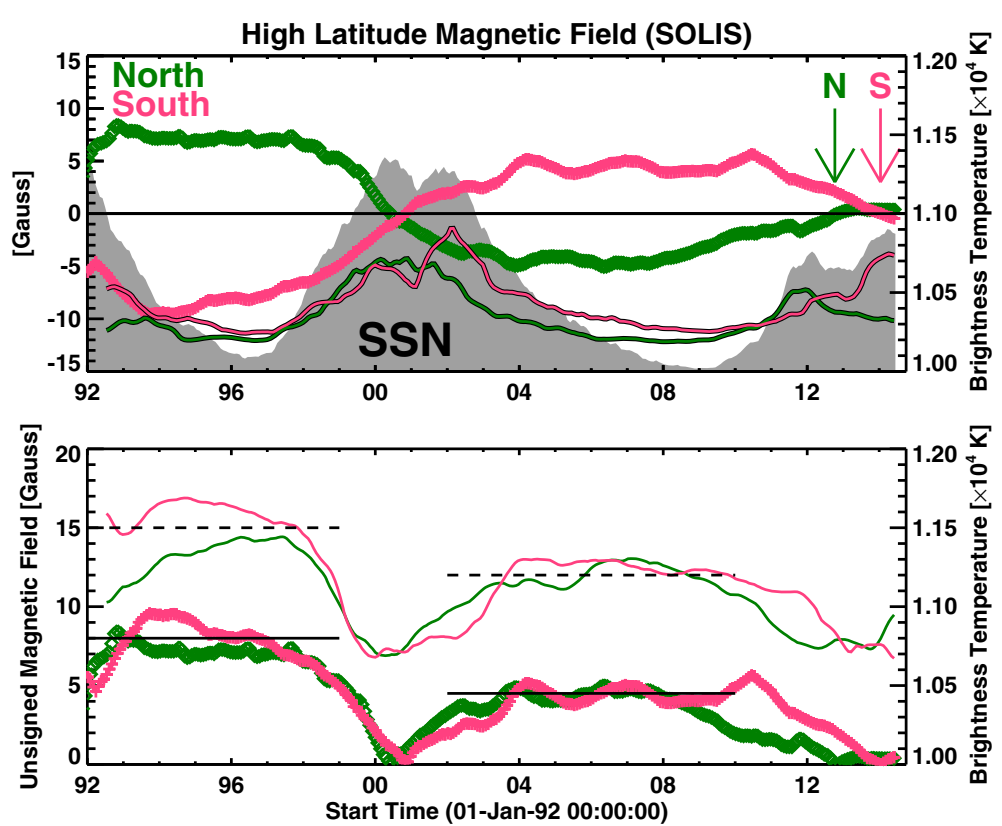

Figure 1 Comparison between solar cycles 23 and 24 from various observations. (Top) An overview of solar cycle 24 with respect to cycle 23 using three sets of observations: the international sunspot number (SSN in gray), polar magnetic field strength in the north and south polar regions ( $B$, averaged over the latitude range $60^{\circ}$ to $90^{\circ}$, thick lines), and the low-latitude microwave brightness temperature (Tb, averaged over 0 to $40^{\circ}$, thin lines). The low-latitude $\mathrm{Tb}$ is due to active regions. $\mathrm{N}$ and $\mathrm{S}$ point to the time when the polar magnetic fields vanished before the sign reversal. (bottom) Polar microwave brightness temperature ( $\mathrm{Tb}$, averaged over the latitude range $60^{\circ}$ to $90^{\circ}$ ) and the unsigned polar magnetic field strength (B). The horizontal dashed and solid lines roughly indicate the average levels of $\mathrm{Tb}$ and $\mathrm{B}$, respectively for the two cycles. The time of vanishing polar B occurs first in the north and then in the south during cycle 23 and 24 . However, the lag is more pronounced in cycle 24. Note that the maximum phase has ended in the northern hemisphere (indicated by the steady increase in polar Tb by the end of 2013). All quantities are smoothed over 13 Carrington rotations.

minima, the polar field strength (B) reaches its peak values and vanishes at maxima, changing sign at the end of maxima (Selhorst et al. 2011; Tsurutani et al. 2011a; Gopalswamy et al. 2012a; Shimojo 2013; Nitta et al. 2014; Mordvinov and Yazev 2014). The maximum phase is indicated by the vanishing polar field strength. The polar B in Figure 1 thus suggests that the maximum phase of cycle 24 is almost over. Note that the arrival of the maximum phase is not synchronous in the northern and southern hemispheres for cycles 23 and 24. (see also Svalgaard and Kamide 2013). The lag in the southern hemisphere is more pronounced in cycle 24. Polar microwave $\mathrm{Tb}$ also declined significantly between the cycle 22/23 and 23/24 minima (Gopalswamy et al. 2012a). During solar maxima, the polar Tb drops to the quiet Sun values (approximately $10^{4} \mathrm{~K}$ ) because the polar coronal holes disappear. It must be pointed out that the southern polar field was stronger during both $22 / 23$ and 23/24 minima; and accordingly, the active region $\mathrm{Tb}$ was higher in the southern hemisphere during the cycle 23 and 24 maxima, indicating a close relationship between the polar field strength during a minimum and the activity strength in the following maximum.

\section{Implications for the solar dynamo}

According to the Babcock-Leighton mechanism of the solar dynamo, the polar field strength of one cycle determines the strength of the next cycle. The so-called polar precursor method of predicting the strength of a solar cycle using the peak polar field strength of the preceding minimum has been fairly accurate (see e.g., Svalgaard et al. 2005; Jiang et al. 2013a; Muñoz-Jaramillo et al. 2013; Zolotova and Ponyavin 2013). Recent discussion on the precursor method can be found in Petrovay (2010) and Pesnell (2014) among others. In addition to the traditional polar field measurements, proxies such as $\mathrm{H}$-alpha synoptic charts (Obridko and Shelting 2008) and the polar microwave Tb (Gopalswamy et al. 2012a) can also be used to predict the strength of the activity cycle. The polar microwave $\mathrm{Tb}$ is exceptionally good because it is highly correlated (correlation coefficient $r=0.86$ ) with the polar field strength: $B=0.0067 \mathrm{~Tb}-70 \mathrm{G}$ (Gopalswamy et al. 2012a).

The enhanced low-latitude Tb between 1997 and 2008 corresponds to the solar activity in cycle 23 representing the toroidal field (see also Selhorst et al. 2014). The enhanced high-latitude Tb between 1992 and 1996 represents the poloidal field. The correlation between 
the high- and low-latitude microwave $\mathrm{Tb}$, averaged over Carrington rotation periods, is shown in Figure 2 using data from cycle 22/23 minimum and cycle 23 maximum. Clearly, the correlation is rather high $(r=0.74$ for the northern hemisphere and 0.82 for the southern hemisphere). However, the correlation plots look very different in the northern and southern hemispheres. The maximum correlation occurs for a lag of 75 rotations in the northern hemisphere (approximately 5.7 years) and 95 in the south (7.2 years in the south). The north-south asymmetry noted before for the arrival of maximum phase is also clear in the correlation plots. Thus, the Nobeyama observations provide a strong observational support to the idea that the poloidal field of one cycle decides the strength of the next cycle (toroidal or sunspot field). Furthermore, the Nobeyama data provides a more detailed time structure (Carrington rotation) compared to those (a solar cycle) used in other studies. This finding for cycle 23 can be tested for cycle 24 when it ends in the next few years.

How do we understand the weak cycle 24? Jiang et al. (2013b) considered several possibilities such as (i) the accuracy of SSN, (ii) sunspot tilt angle variation, and (iii) the variation in the meridional circulation during cycle 23. They were able to reproduce the lower polar field during the cycle $23 / 24$ minimum using a $55 \%$ increase of the meridional flow in their model. They also found that a $28 \%$ decrease of the mean tilt angle of sunspot

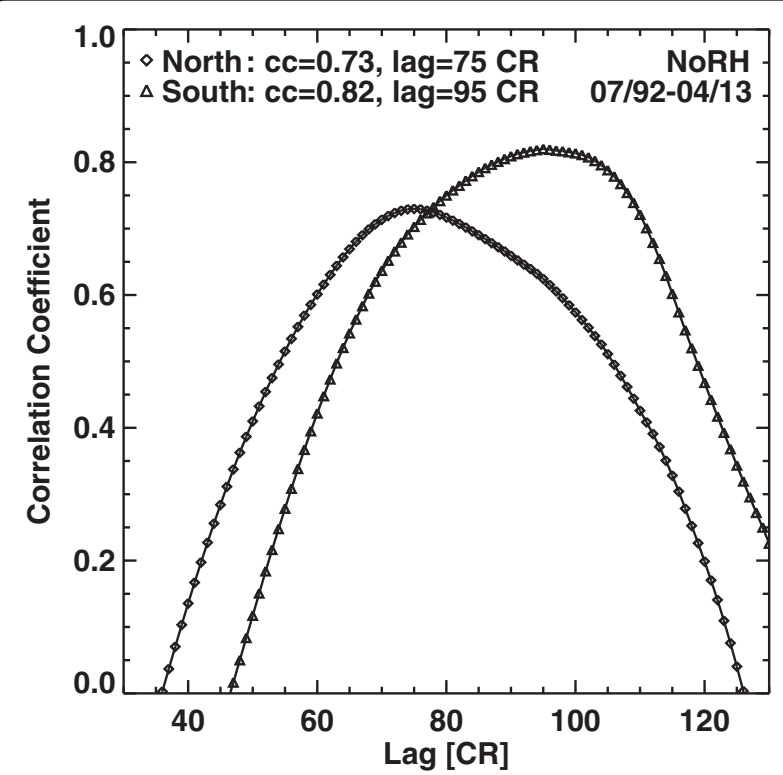

Figure 2 Polar field of cycle 23 and activity strength in cycle 24. Correlation between the polar microwave brightness (proxy to the poloidal field strength of the Sun) of one solar cycle and the low-latitude brightness (proxy to the solar activity) of the next as a function of the time lag in units of Carrington rotation (CR). The correlation is quite high and supports the flux transport dynamo model. The correlation coefficients are shown on the plot for the northern and southern hemispheres along with the lag extent (in number of CRs). groups can explain the low polar field, but this would not be consistent with the observed time of polar field reversals. They concluded that the nonlinearities in the polar field source parameters and in the transport parameters play important roles in the modulation of the polar field.

\section{Implications for the long-term behavior of the Sun}

The Sun is known to have variability on time scales up to millennia (see Usoskin 2013 for a review). One obvious question is whether the weakening of the activity observed in cycle 24 will continue further. Javaraiah (2015) examined the north-south asymmetry of sunspot areas binned into $10^{\circ}$ latitudes and examined various periodicities. They found periodicities of 12 and 9 years, respectively, during low-activity (1890 to 1939) and highactivity (1940 to 1980) intervals. They also inferred that cycle 25 may be weaker than cycle 24 by approximately $31 \%$. Several authors have discussed the possibility of a global minimum over the next several cycles (see e.g., Russell et al. 2013a; Lockwood et al. 2011; Steinhilber and Beer 2013; Zolotova and Ponyavin 2014; Ruzmaikin and Feynman 2014). Zolotova and Ponyavin (2014) reported that the protracted cycle 23 is similar to the cycles immediately preceding the Dalton and Gleissberg-Gnevyshev minima, suggesting that the Sun is heading toward such a grand minimum.

But the most important is that the diminished solar activity has immediate consequences for the society. When the Maunder Minimum occurred in the late 1600 s, the technology was not seriously affected by the Sun. Today's technology is extensively coupled to solar activity, so the effect is readily recognized. For example, the weak solar activity has resulted in reduced atmospheric drag on satellites increasing their lifetime. On the other hand, space debris do not burn up quickly, thus posing additional danger to the operating satellites. The geomagnetic disturbances have been extremely mild, with the weakest level of geomagnetic storms since the space age.

\section{The weakest geomagnetic activity on record: cycle 23 minimum}

Figure 3 shows from top to bottom: the sunspot number $(\mathrm{Rz})$, the $1 \mathrm{AU}$ interplanetary magnetic field magnitude (Bo), the Oulu Finland cosmic ray count rate (the local vertical geomagnetic cutoff rigidity is approximately 0.8 $\mathrm{GV}$ ), the solar wind speed (Vsw), and the ap geomagnetic index. The vertical dashed green lines give the official dates of the solar minima between cycles 22 and 23 and cycles 23 and 24 (Hathaway 2010). The vertical blue lines give the geomagnetic ap index minima. The horizontal red lines have been added to the figure to guide the reader. From top to bottom, the lines are the zero value for $\mathrm{Rz}, 5 \mathrm{nT}$ for Bo, $6,500 \mathrm{cts} / \mathrm{min}$ for the cosmic ray flux, $400 \mathrm{~km} / \mathrm{s}$ for 


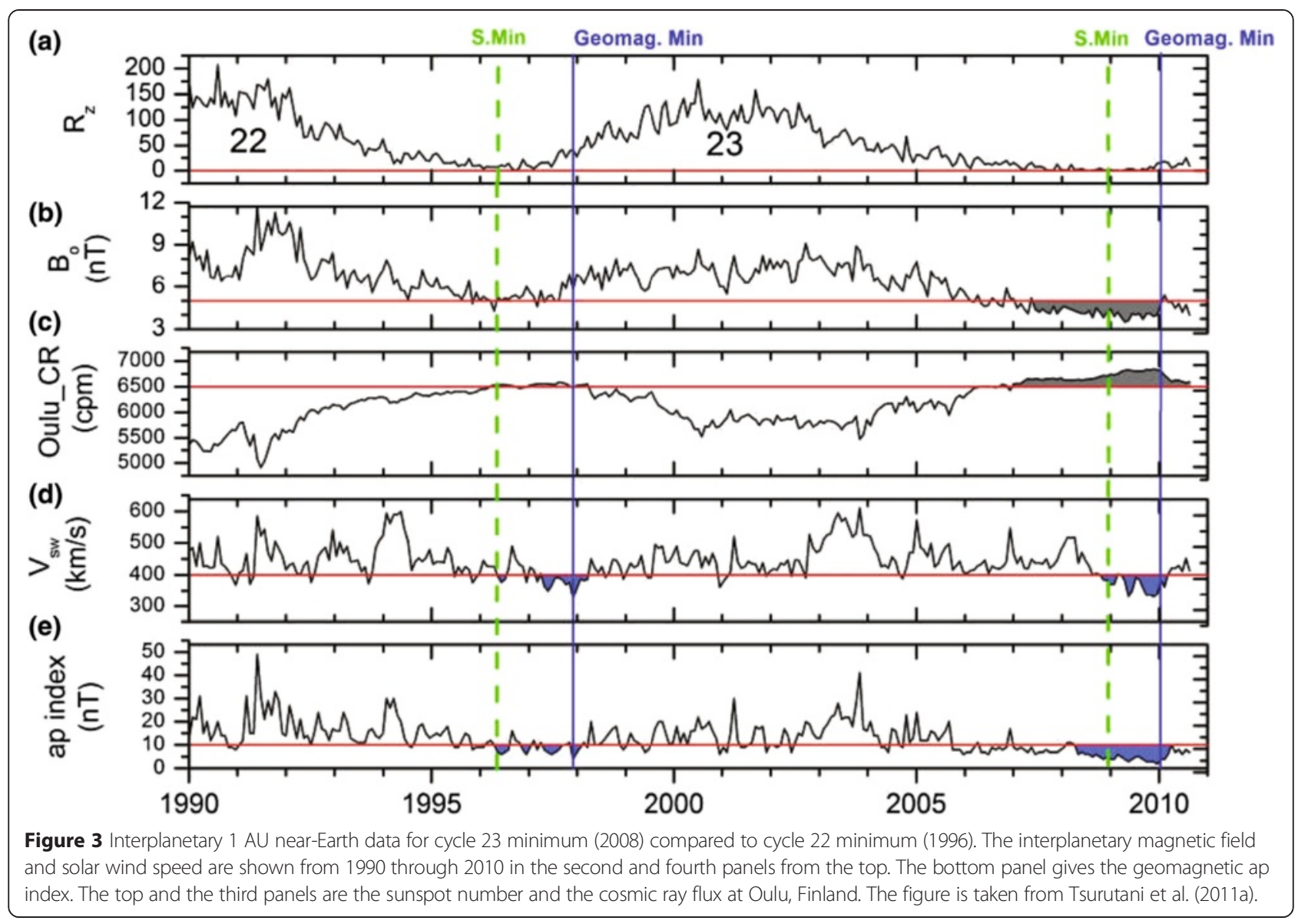

Vsw, and $10 \mathrm{nT}$ for ap. We call the reader's attention to the long delay between the sunspot minima and the geomagnetic activity minima. This occurs in both solar cycle minima. The 2010 geomagnetic ap minimum is the lowest since the index began to be recorded.

The figure shows that cycle 23 extended from 1996 to 2008 and is the longest in the space era (12.6 years). For comparison, the length of solar cycles 20 through 22 was $11.7,10.3$, and 9.7 years, respectively. The values below the red lines have been shaded for emphases (in the case of cosmic rays, the values above the red line are shaded). It can be noted that the Bo, Vsw, and ap index values for the cycle 23 minimum are considerably lower than the cycle 22 minimum values. The minimum in ap is broad and extends from day 97, 2008, until day 95, 2010. The onset and end times are somewhat arbitrary. There is a minimum geomagnetic activity interval in cycle 22 (day 106, 1996, to day 23, 1998).

Figure 4 shows the solar wind, the IMF magnetic field magnitude, the interplanetary epsilon parameter (Perrault and Akasofu 1978), and the ap index from 2008 through the first part of 2010. This interval is noted for a general lack of CMEs (and magnetic storms) and the dominance of high-speed streams (top panel).
What is unusual about this is the general decline in the peak solar wind speed starting in 2008 and extending to 2010. The peak solar wind speeds of high-speed streams are typically 750 to $800 \mathrm{~km} / \mathrm{s}$ at $1 \mathrm{AU}$ and beyond (Tsurutani et al. 1995; Tsurutani and Ho 1999), but here, none of these streams have these magnitudes.

What is the cause of this extremely low geomagnetic activity between cycle 23 and cycle 24? It was found that coronal holes during this phase of the solar cycle are small and located near middle latitudes (De Toma 2011). This caused the solar wind speed from coronal holes to be weak and the magnetic field variances to be particularly low (not shown). A schematic to indicate all of these features are shown in Figure 5.

It is surmised that nothing has changed on the speed of the high-speed streams emanating from coronal holes during solar minimum. The terminal speed is still approximately 750 to $800 \mathrm{~km} / \mathrm{s}$. However, this is the speed for the central portion of the hole. As the high speed stream expands into interplanetary space, it does not simply propagate radially outward but expands into nearby space, leading to 'super-radial' expansion as shown in the schematic of Figure 5. At the sides of the high-speed stream, the speed and the amplitude 


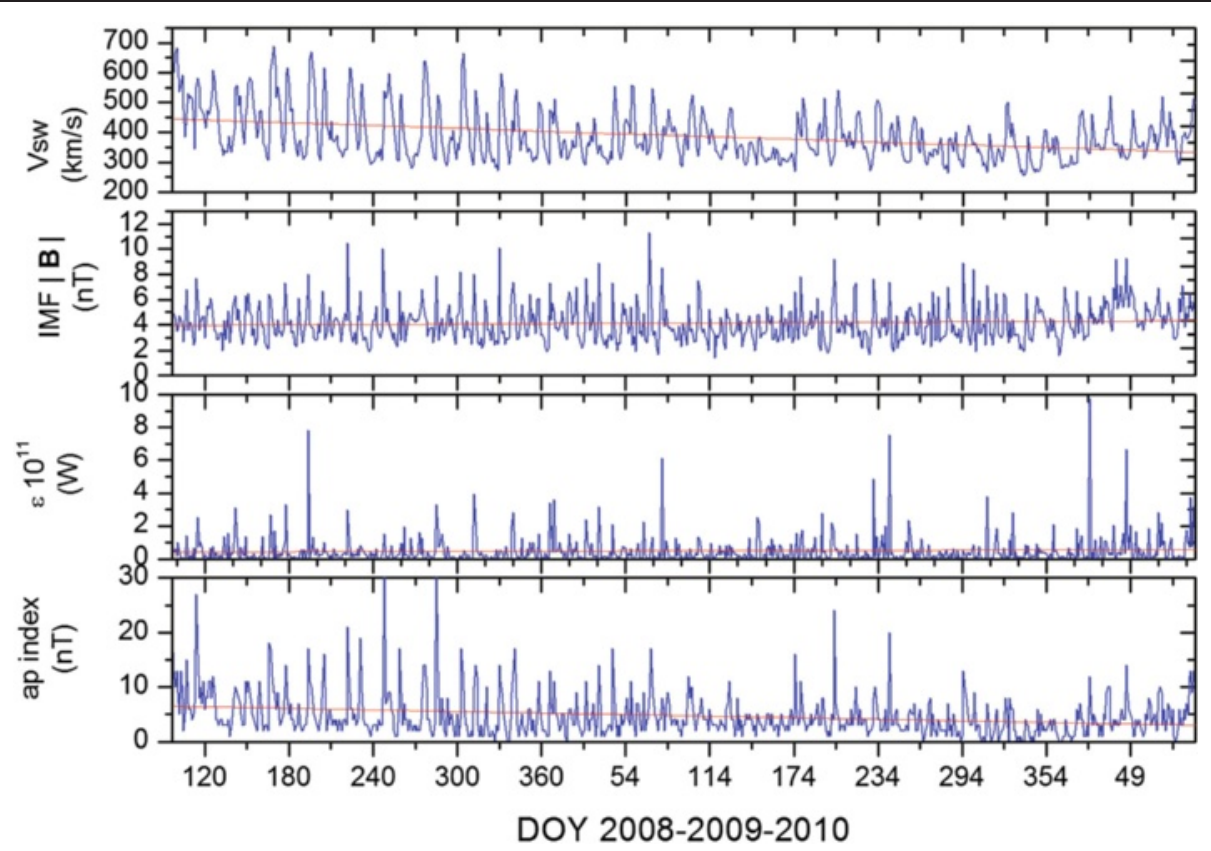

Figure $4 \mathrm{~A}$ blow-up of the $1 \mathrm{AU}$ interplanetary parameters and the ap index.

of the entrained Alfvén waves will be reduced. This is the portion of the high-speed streams that hit the Earth's magnetosphere.

Thus, the low solar magnetic fields, the lack of CMEs, and the midlatitude location of small coronal holes all contribute to the all-time minimum in the geomagnetic activity between 2008 and 2009. It is noted that in Figure 3, a similar feature can be noted in the cycle 22 minimum, but the feature is less prominent.

\section{Coronal mass ejections and flares Origin of solar eruptions}

Although it is well established that CMEs and their interplanetary manifestations, ICMEs, and flares originate from closed-field regions on the Sun such as active regions and filament regions, the current level of understanding is not sufficient to predict when an eruption might occur in such a region. Two basic processes seem to be involved: energy storage and triggering. The energy storage can be identified from non-potentiality of the source region such as magnetic shear or accumulated helicity (Tsurutani et al. 2009a; Kazachenko et al. 2012). Zhang et al. (2012a) studied the magnetic helicity of axisymmetric power-law force-free fields and focused on a family whose surface flux distributions are defined by self-similar force-free fields. The results suggest that there may be an absolute upper bound on the total magnetic helicity of all bipolar axisymmetric force-free fields.

In addition to the energy storage, a trigger in the form of a magnetic disturbance seems to be necessary, which causes a pre-eruption reconnection (Kusano et al. 2012).
These authors suggest that observing these triggers is important for predicting eruptions and that we can predict eruptions only by a few hours before the eruption. For longer-term predictions, one has to resort to probabilistic methods. Huang et al. (2011) presented a study of a coronal mass ejection (CME) with high temporal cadence observations in radio and extreme-ultraviolet (EUV). The radio observations combined imaging of the low corona with radio spectra in the outer corona and interplanetary space. They found that the CME initiation phase was characterized by emissions that were signatures of the reconnection of the outer part of the erupting configuration with surrounding magnetic fields. Later on, a main source of emission was located in the core of the active region, which is an indirect signature of the magnetic reconnection occurring behind the erupting flux rope. Energetic particles were also injected in the flux rope and the corresponding radio sources were detected. Other radio sources, located in front of the EUV bright front, traced the interaction of the flux rope with the surrounding fields. They found that imaging radio emissions in the metric range can trace the extent and orientation of the flux rope which was later detected in interplanetary space.

\section{Long-term behavior of CME rates}

Although CMEs were discovered in 1971 (Tousey 1973), understanding their long-term behavior became possible only after the launch of the Solar and Heliospheric Observatory (SOHO). The continuous observations from the Large Angle and Spectrometric Coronagraph (LASCO) on board SOHO since 1996 constitute a 


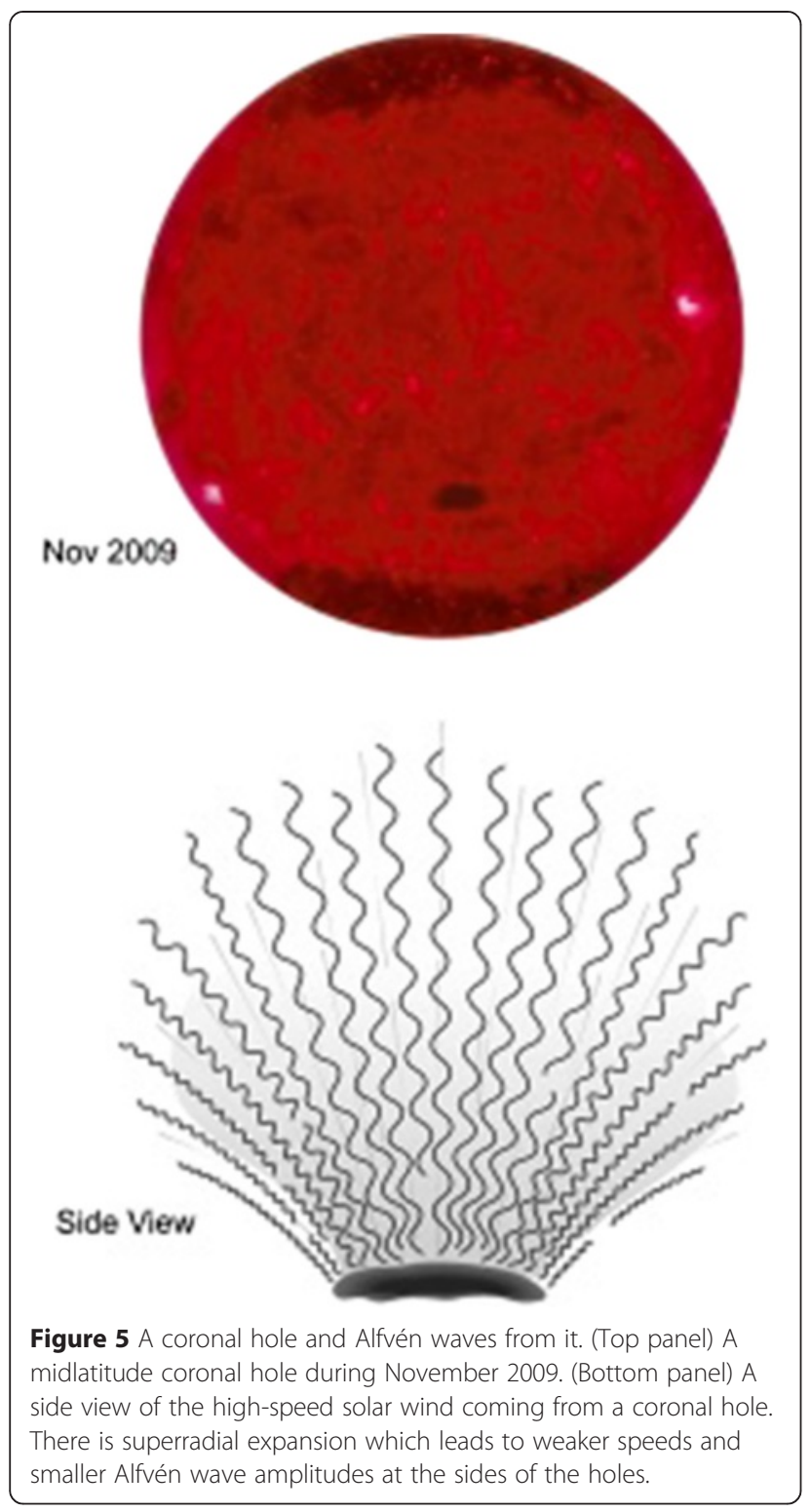

uniform and extended data set on CMEs. Figure 6 shows a plot of the CME rate and speed averaged over Carrington rotation periods (27 days) for cycles 23 and 24, including the prolonged minimum between the two cycles. Only CMEs of width $30^{\circ}$ or larger have been included in the plots; including narrower CMEs would increase the rate even higher in cycle 24 . We used the width criterion to avoid variability due to manual identification by different people and the change in SOHO/LASCO image cadence in 2010. We see that the CME rate over the first 5 years in each cycle is not drastically different, even though the sunspot number dropped significantly. The prolonged cycle 23/24 minimum had low CME rate (but non-zero) similar to the cycle $22 / 23$ minimum. The average CME speed decreased significantly during the $23 / 24$ minimum compared to that during the $22 / 23$ minimum. However, the average speeds during the cycle 23 and 24 maxima were not significantly different.

Figure 7 shows a detailed comparison between the corresponding epochs of cycles 23 and 24. The SSN averaged over the first 5 years in each cycle dropped from 68 to 38, which is a $44 \%$ reduction in cycle 24 . On the other hand, the CME rate remained the same (2.09 in cycle 23 vs. 2.10 in cycle 24). This means that the relation between SSN and CME rate changed in cycle 24 (the daily CME rate per SSN is greater in cycle 24), which will be discussed in 'Coronal mass ejections and flares' section. There is ongoing debate to understand the reason for this difference: possible artifacts (Wang and Colaninno 2014; Lamy et al. 2014), changing strength of the poloidal field (Petrie 2012, 2013), or the altered state of the heliosphere (Gopalswamy et al. 2014a).

\section{Importance of CMEs for space weather}

For space weather effects, more energetic CMEs need to be examined. Gopalswamy et al. (2014b) started with flares of soft X-ray size $\geq$ C3.0. This criterion avoids the effect of soft X-ray background level and its variability between the two cycles. For example, approximately $20 \%$ of flares of size $<\mathrm{C} 1$ cannot be detected by GOES during cycle 23 , while the corresponding fraction is $6 \%$ for cycle 24 . However, for flares of size $\geq$ C 3.0 , no flares go undetected. The source locations of flares were obtained from the online Solar Geophysical Data (SGD) or identified using nearsurface observations from a number of sources: EUV images from SOHO, STEREO (Solar Terrestrial Relations Observatory), and SDO (Solar Dynamics Observatory), soft X-ray images from Yohkoh, microwave images from the Nobeyama Radioheliograph, and $\mathrm{H}$-alpha images from various observatories. For each of these flares, the association of a CME was checked using the SOHO/LASCO catalog (http://cdaw.gsfc.nasa.gov, Gopalswamy et al. 2009a) to compile the properties of the associated CMEs. Only flares that occurred within $30^{\circ}$ from the limb were considered, so that the speed and width measurements of CMEs were subject to minimal projection effects.

Figure 8 shows histograms of soft X-ray flares binned into various classes. Clearly, the number of flares with CME association depends on the flare size as is well known (Yashiro et al. 2005). The mean and median flare sizes are roughly the same in both cycles, and the shape of the distributions are quite similar. The total number of flares of size $\geq$ C 3.0 was 664 and 554 for cycles 23 and 24 , respectively over the first 62 months in each cycle. This corresponds to a reduction of approximately $17 \%$ in cycle 24. This reduction is not as large as the drop in SSN. The number of flares with CMEs was 273 for cycle 23 compared to 214 in cycle 24 , which corresponds to a reduction of approximately $22 \%$ from cycle 23 to 24 . 

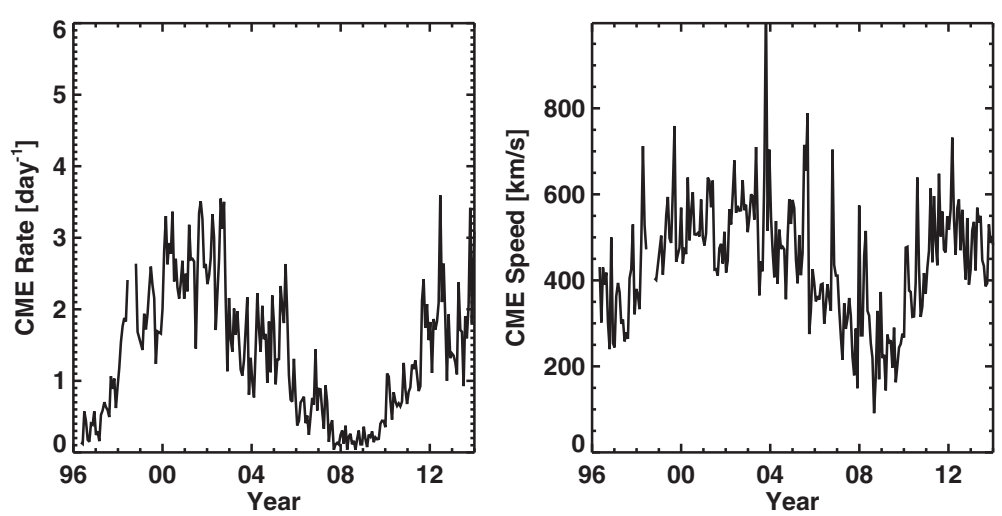

Figure 6 CME occurrence rate (per day) and speed in cycles 23 and 24 (1996 to 2013). The quantities have been averaged over Carrington rotation periods ( 27 days). Only CMEs with width $\geq 30^{\circ}$ are included in the plot. The CME information is from the SOHO/LASCO CME catalog.

Accounting for the lack of CME data for about 4 months in cycle 23 when $\mathrm{SOHO}$ was temporarily disabled, the reduction was $27 \%$. Again, the decline in the CME rate was not as drastic as the SSN. However, this is different from the same average CME rate found for cycles 23 and 24 (Figure 7) suggesting that the reduction was in the number of energetic eruptions.

\section{CME speed and width distributions}

The properties of the CMEs in the two cycles are compared in Figure 9. The speed distributions in the two cycles were similar with almost the same average (633 vs. $614 \mathrm{~km} / \mathrm{s}$ ) and median ( $514 \mathrm{~km} / \mathrm{s}$ vs. $495 \mathrm{~km} / \mathrm{s}$ ) speeds. On the other hand, the width distributions were significantly different: the average and median widths of
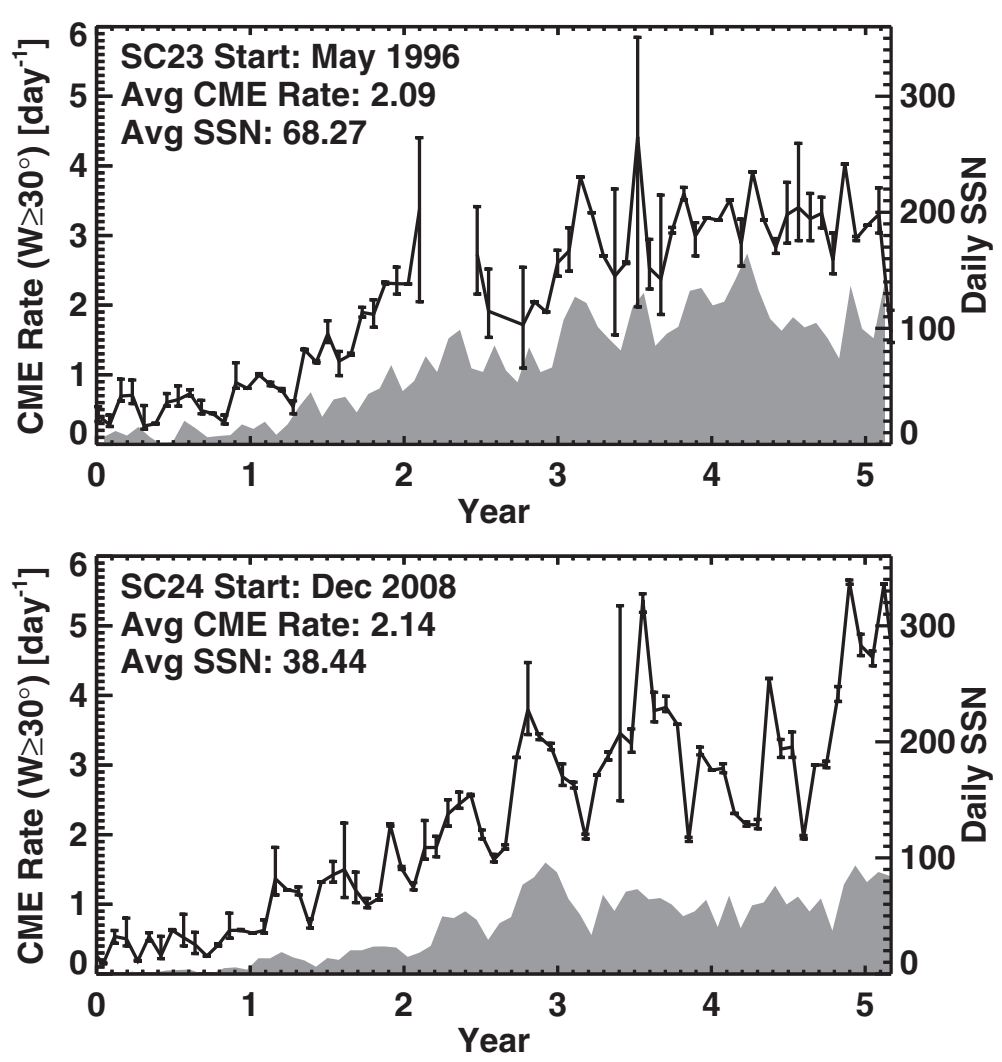

Figure 7 Detailed comparison between the corresponding epochs of cycles 23 and 24 (approximately 5 years). Daily SSN (International) is included in each case. The average CME rates averaged over the first 5 years in each cycle are shown on the plots. The error bars are based on SOHO down times $\geq 3 \mathrm{~h}$, which are listed in the SOHO/LASCO CME catalog. 

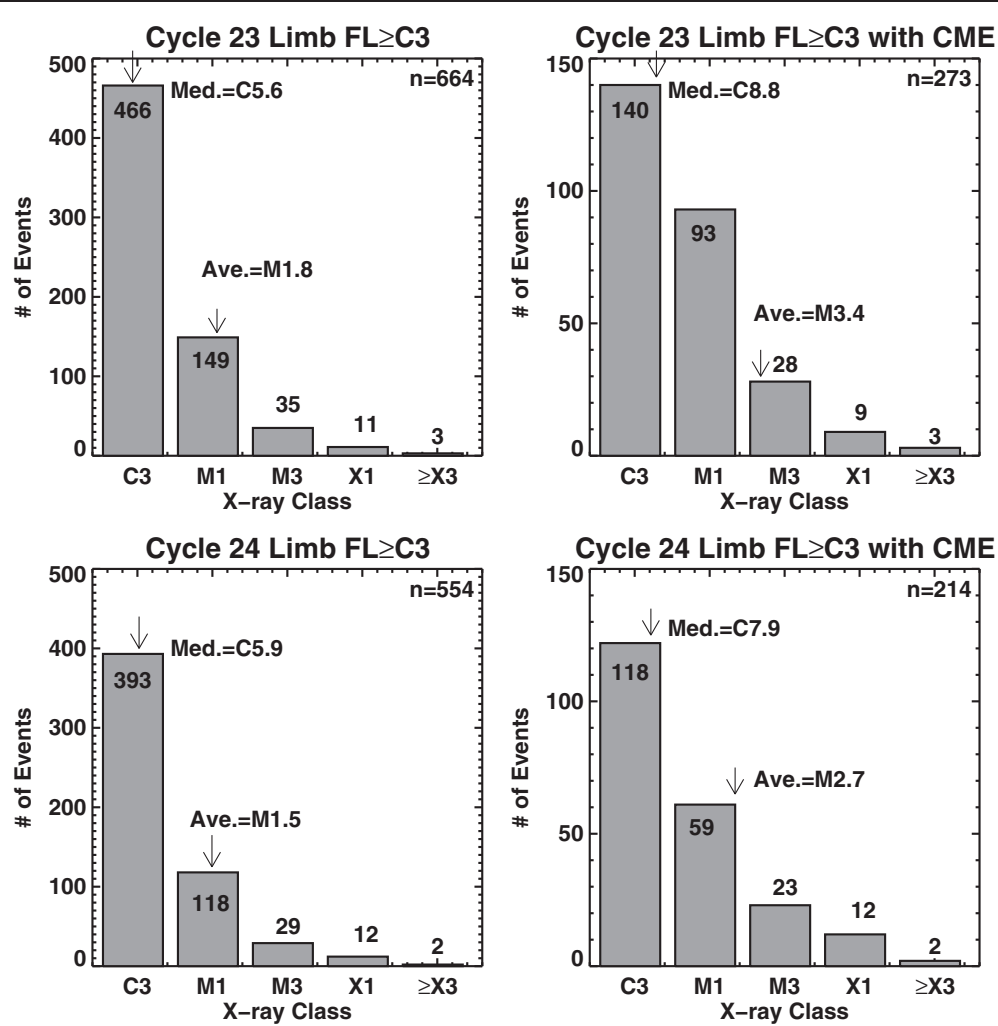

Figure 8 Distributions of flares originating within $30^{\circ}$ from the limb for cycle 23 and 24. (Left) All flares and (right) flares associated with CMEs. The average (Ave) and median (Med) values of the distributions are marked on the plots.

non-halo CMEs in cycle 24 were much higher than those in cycle 23 over the corresponding epoch. The fraction of halo CMEs in cycle 23 was $3 \%$, which is typical of the general population of CMEs (Gopalswamy et al. 2010a). However, the halo fraction was $9 \%$ in cycle 24 , three times larger than that in cycle 23.

The overabundance of cycle-24 halo CMEs was also observed in the general population. Halo CMEs are socalled because new material is observed all around the occulting disk in sky-plane projection (Howard et al. 1982; Gopalswamy et al. 2010a). Figure 10 shows the distribution of halo CMEs (binned over Carrington rotation periods) as a function of time. There were 199 halo CMEs (apparent width $=360^{\circ}$ ) during cycle 24 until the end of April 2014, amounting to approximately 3.06 CMEs per month. On the other hand, there were only 178 halos during the first 65 months of cycle 23 or 2.99 CMEs per month (adjusting for 4 months of $\mathrm{SOHO}$ was not observed in cycle 23). Clearly, the halo CME occurrence rate in cycle 24 did not decrease at all (see Gopalswamy et al. 2015 for more details). For a given coronagraph, halo CMEs represent fast and wide (and hence energetic) CMEs (Gopalswamy et al. 2010b), further suggesting something peculiar about CME widths in cycle 24.

Table 1 compares the number of CMEs in cycles 23 and 24 under various categories (CME width, width and speed, and flare size). 'All CMEs' includes every CME that was identified and measured. The largest difference was for the narrowest CMEs (width $(W)<30^{\circ}$ ): the number of narrow CMEs in cycle 24 was a factor of 2 higher, which was the reason for the difference in the general population. These CMEs are generally not well defined and do not travel far from the Sun. There may be multiple reasons for the overabundance of narrow CMEs including measurement bias and the change in image cadence. The number of CMEs in cycles 23 and 24 became equal for wider CMEs. For CMEs with width $\geq 60^{\circ}$, the monthly rate was roughly the same in the two cycles. This was also true for halo CMEs. Fast and wide CMEs, on the other hand, showed a decline in number in cycle 24 as did CMEs associated with $\geq$ C 3.0 flares.

\section{Anomalous expansion of CMEs in cycle 24}

The speed vs. width scatterplot in Figure 11 further illustrates the different width distribution in cycle 24 (Gopalswamy et al. 2014a). The plot confirms the wellknown linear relationship between speed and width of CMEs (faster CMEs are wider), but the slope is significantly larger in cycle 24 . This means that the cycle-24 CMEs are significantly wider for a given speed. This also explains the high abundance of halo CMEs in cycle 24 


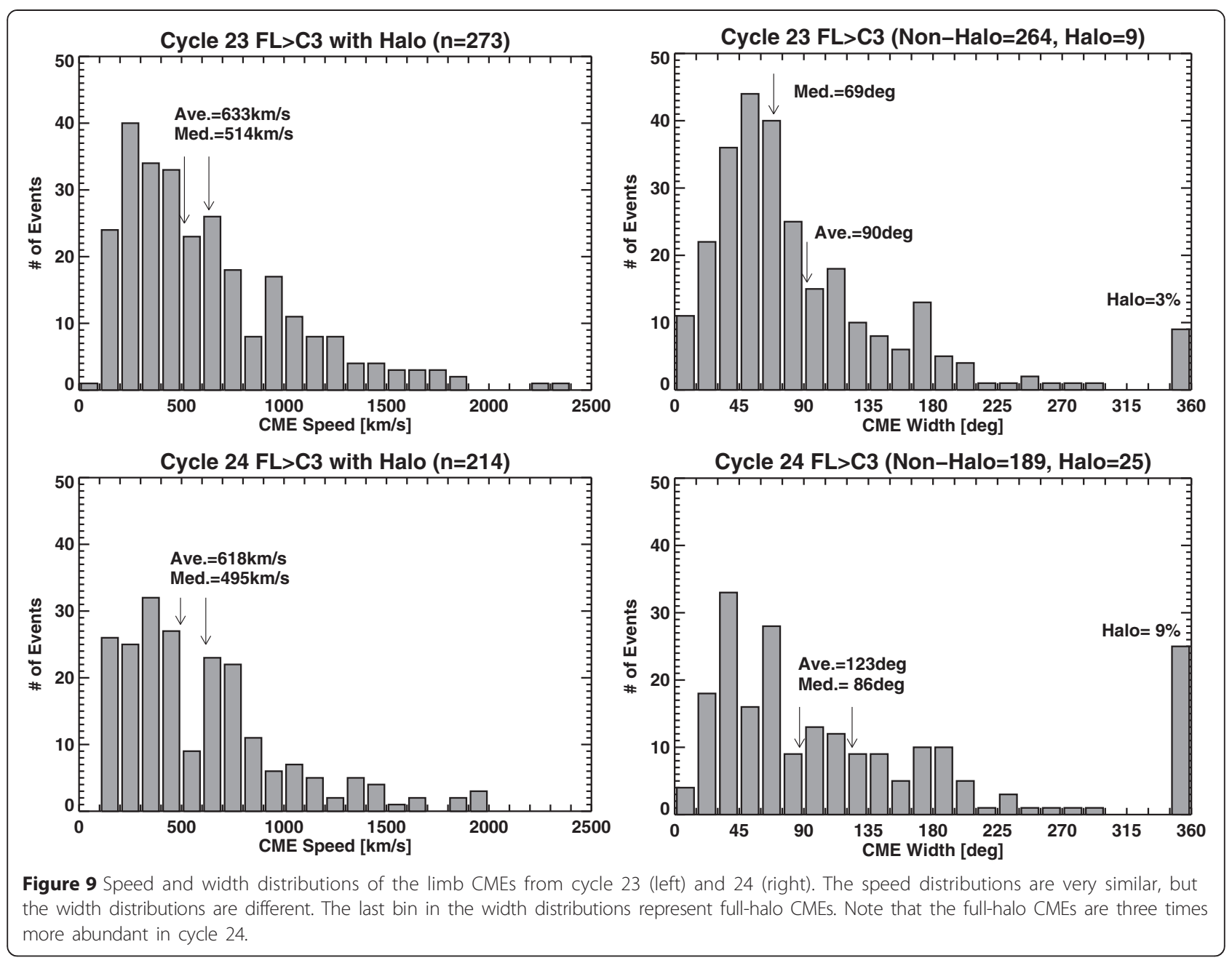

even though there is a slight reduction in the number of energetic CMEs in this cycle.

\section{CME mass distribution in cycles 23 and 24}

CME mass can be determined from LASCO images and is thought to be accurate within a factor of approximately 2. Since we are considering limb CMEs, the projection effects are minimal and the mass estimate is expected to be more accurate. The average masses shown in Figure 12 is over the first 62 months of cycle 23 and are generally consistent with previous estimates (Gopalswamy et al. 2010b; Vourlidas et al. 2011). However, the cycle-24 CME masses are smaller by a factor of approximately 3 (see Figure 12). The cycle-24 value is also smaller than the mass averaged over the entire solar cycle 23 (Vourlidas et al. 2011). Gopalswamy et al. (2005a) used more than 4,000 CMEs during 1996 to 2003 and found that the CME mass $(M)$ and width $(W)$ were correlated $(r=0.63)$ with a regression equation: $\log M=1.3$ $\log \mathrm{W}+12.6$. This relationship is also true for the limb CMEs used in Figure 11: $\log M=1.54 \log \mathrm{W}+12.4$ (cycle 23) and $\log M=1.84 \log \mathrm{W}+11.5$ (cycle 24). The slope of the cycle-24 regression line is slightly larger. For a $\mathrm{CME}$ of approximately $60^{\circ}$ width, the cycle-23 CME mass was larger by a factor of approximately 2.4. This result is consistent with the anomalous expansion of cycle-24 CMEs: a $60^{\circ}$ wide CME in cycle 24 is equivalent to a narrower CME in cycle 23.

\section{The weak state of the heliosphere}

The inflated CME size in cycle 24 seems to be a direct consequence of the weak heliosphere, stemming from the weaker activity at the Sun. The physical parameters of the heliosphere all showed smaller values in the rise to the maximum phase of cycle 24. McComas et al. (2013) extended their earlier work (McComas et al. 2008) on the extended cycle 23/24 minimum to the rise phase of cycle 24 and shown that the solar wind densities, proton temperatures, dynamic pressures, and interplanetary magnetic field strengths were all diminished. Even the density fluctuations in the slow solar wind diminished significantly (Tokumaru et al. 2013). The effect was even 


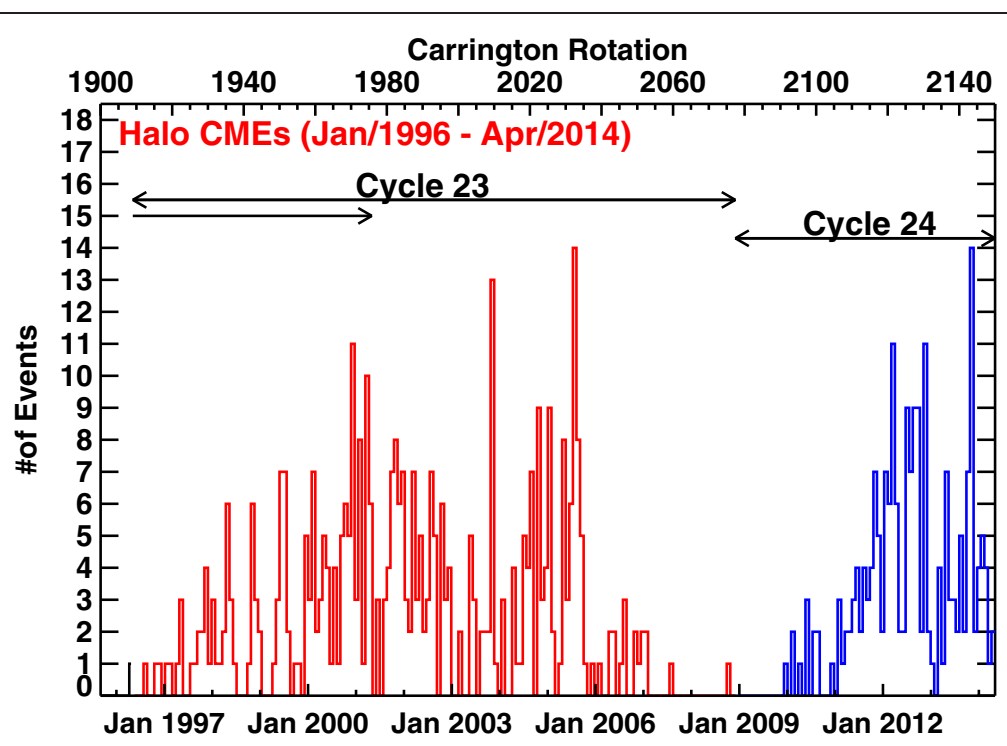

Figure 10 The occurrence rate of halo CMEs from 1996 to the end of 2013. The occurrence rate of halo CMEs (number per Carrington rotation) from 1996 to the end of 2013 detected by SOHO/LASCO. From 1 December 2008 to 31 December 2013, there were 186 halo CMEs. Over the corresponding phase in cycle 23 (10 May 1996 to 9 June 2001), there were only 162 halos. If the halos occurred with the same average rate during the 4-month period when $\mathrm{SOHO}$ was operational, the number is expected to be approximately 173 . Thus, the number of full halos in cycle 24 is comparable to that of cycle 23 or slightly greater (data from Gopalswamy et al. 2015).

felt at the heliospheric termination shock, whose size decreased by approximately 10 AU (J. Richardson, 2014, private communication). Gopalswamy et al. (2014a) reported that the total pressure (magnetic + kinetic) pressure, magnetic field, and the Alfvén speed all declined significantly in cycle 24 . Figure 13 compares several solar wind parameters measured at $1 \mathrm{AU}$ and the same values extrapolated to the vicinity of the Sun. The reduced heliospheric pressure can readily explain the inflated CMEs in cycle 24. The drastic change in the state of the heliosphere between cycles 23 and 24 has important implications for space weather events (see later).

\section{Forbush decrease}

Forbush decrease (FD) represents the reduction in the intensity of galactic cosmic rays (GCRs) as detected by neutron monitors and muon detectors due to solar wind

Table 1 Comparison between CME numbers in solar cycles 23 and 24

\begin{tabular}{lllc}
\hline CME property & Cycle 23 $^{\mathbf{a}}$ & Cycle 24 & Ratio $^{\mathbf{b}}$ \\
\hline All CMEs & $5,086(89.2 / \mathrm{mo})$ & $8,201(134.44 / \mathrm{mo})$ & 1.51 \\
$\mathrm{~W}<30^{\circ}$ & $1,732(30.38 / \mathrm{mo})$ & $3,907(64.05)$ & 2.11 \\
$\mathrm{~W} \geq 30^{\circ}$ & $3,354(58.84 / \mathrm{mo})$ & $4,294(70.39 / \mathrm{mo})$ & 1.20 \\
$\mathrm{~W} \geq 60^{\circ}$ & $1,858(32.6 / \mathrm{mo})$ & $2,205(36.15 / \mathrm{mo})$ & 1.13 \\
$W=360^{\circ}$ & $178(2.99 / \mathrm{mo})$ & $199(3.06 / \mathrm{mo})$ & 1.02 \\
$\mathrm{~V} \geq 900 \mathrm{~km} / \mathrm{s}$ and $\mathrm{W} \geq 60^{\circ}$ & $189(3.32 / \mathrm{mo})$ & $142(2.33 / \mathrm{mo})$ & 0.70 \\
$\geq$ C3.0 flares, limb & $273(4.7 / \mathrm{mo})$ & $214(3.45 / \mathrm{mo})$ & 0.73 \\
\hline
\end{tabular}

aver the same epoch as cycle 24 . ${ }^{\mathrm{b}}$ Ratio of cycle-24 rate to cycle-23 rate. disturbances (see e.g., Munakata et al. 2005; Dumbovic et al. 2012; Arunbabu et al. 2013; Ahluwalia et al. 2014; Belov et al. 2014). Both CMEs and CIRs cause a FD, but the amplitude is significantly higher for CMEs than for CIRs (Dumbovic et al. 2012; Maričić et al. 2014). FD is one of the beneficial effects of solar activity in that the impact of GCRs on Earth is moderated by Earth-directed CMEs. Belov et al. (2014) investigated FDs making use of the CME database from SOHO and GCR intensity from the worldwide neutron monitor network. They found good correlations of the FD magnitude with the CME initial speed, the ICME transit speed, and the maximum solar wind speed. Full-halo CMEs showed the maximum FD, followed by partial halos and non-halos. Figure 14 shows that faster and wider CMEs are more effective in causing FDs. Note that the CMEs in the $360^{\circ}$ bin are most effective in causing FD. Full-halo CMEs generally originate close to the disk center and head directly toward Earth and hence are effective in producing FDs and geomagnetic storms. These results are consistent with the findings by Abunina et al. (2013), who found that the solar sources of disturbances causing the maximum FD are close to the central meridian (E15 to W15).

Despite large international efforts in understanding FDs, there is still a lot to learn. The current model of FDs consisting of two-step decrease has recently been questioned. It is not clear if only a subset of CMEs originating from the disk center is effective in causing FDs (Jordanova et al. 2012). However, the study of FDs has been gaining interest in recent times because of the 


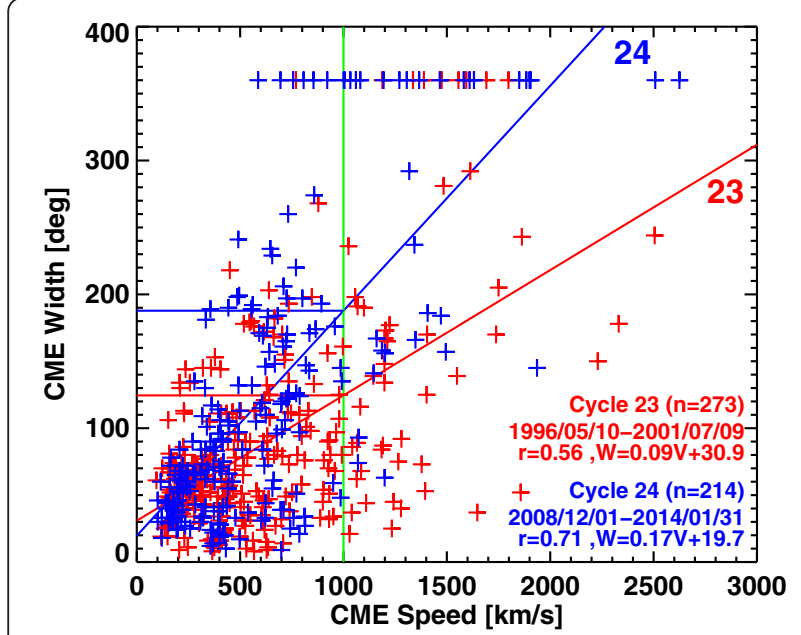

Figure 11 Speed vs. width distributions of limb CMEs from cycles 23 and 24. Both cycles show a good correlation between speed and width, but the slopes are very different. The correlations coefficients $(r)$ and the regression lines are given on the plot. Student's $t$-test confirms that the slope difference is statistically significant. The data points at width $=360^{\circ}$ are halo CMEs, which are mostly from cycle 24 .

space weather applications. For example, the development of Global Muon Detector Network (GMDN - Munakata et al. 2005; Fushishita et al. 2010; Rockenbach et al. 2011) has greatly enhanced the possibility of forecasting ICME arrival using the network (see e.g., Rockenbach et al. 2014 for a review).

\section{Spatial structure of CMEs}

Even before the discovery of white-light CMEs, the concept of magnetic loops from the Sun driving shocks was considered (Gold 1962). In Gold's picture, a magnetic bottle from the Sun drives a fast magnetosonic shock which stands at certain distance from the bottle. Such a shock was first identified by the Mariner 2 mission in 1962 (Sonett et al. 1964). Koomen et al. (1974) identified white-light CMEs with the Gold bottle. Burlaga et al. (1981) confirmed the basic picture of Gold using in situ data by identifying the shock, sheath, and the driving magnetic structure. Near the Sun, MHD shocks were inferred from metric type II radio bursts for several decades ago (see e.g., Nelson and Melrose 1985). The overall CME structure consisting of a flux rope enclosing a prominence core and driving a shock outside has been considered by theorists a while ago (e.g., Kuin and Martens 1986), but it took another two decades before the white-light shock structure of CMEs was observed in coronagraphic images (Sheeley et al. 2000). A recent study based on coronagraph observations concluded that a flux rope structure can be discerned in approximately $40 \%$ of CMEs observed near the Sun (Vourlidas et al. 2013).

\section{White-light and EUV signatures of CME-driven shocks}

Fast forward interplanetary shocks (hereafter simply called 'shocks') are driven by either fast CMEs or high-speed
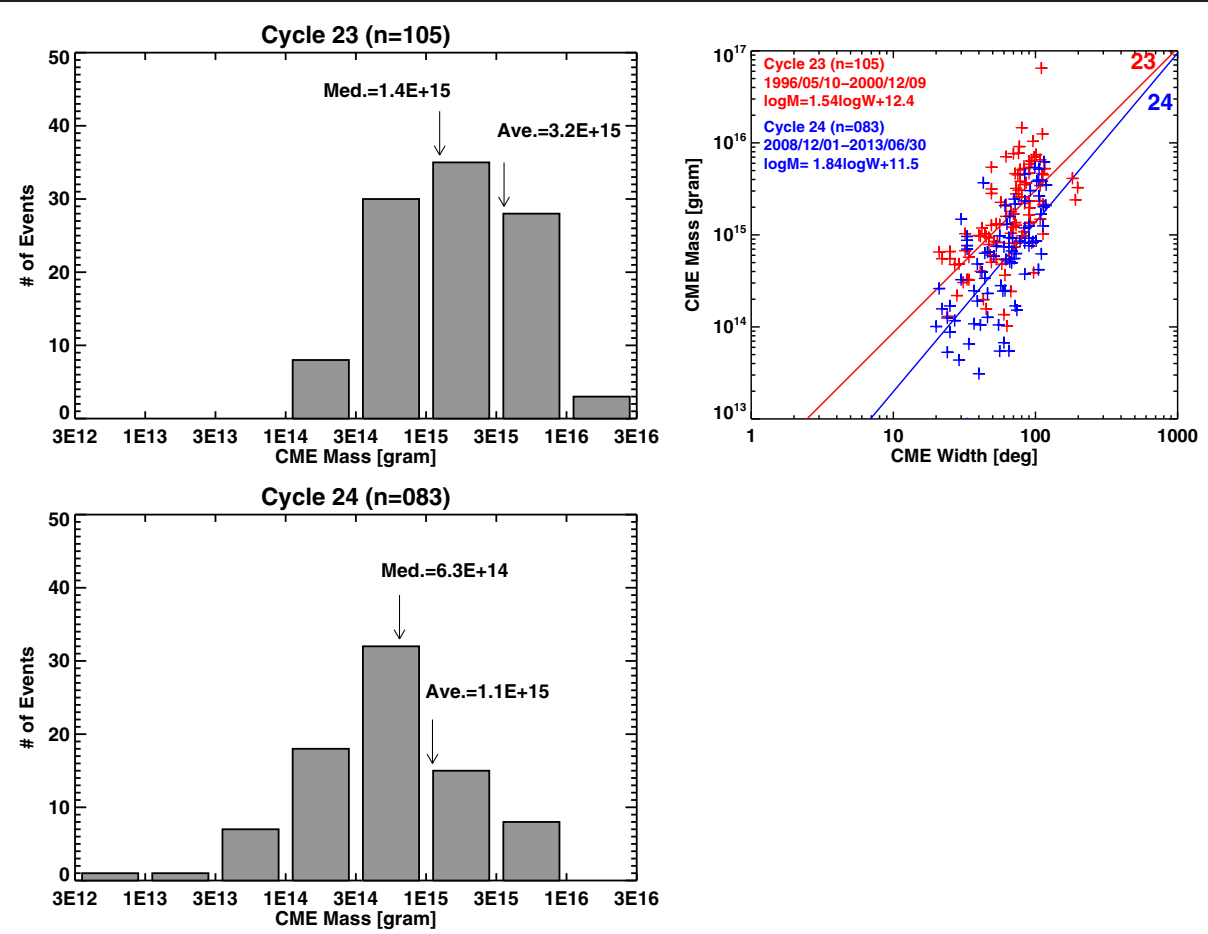

Figure 12 CME mass distributions and mass-width plots. (Left) CME mass distributions for limb CMEs from cycle 23 and 24. (Right) Mass-width plots for the two cycles. For a given CME width, the mass is larger for cycle-23 CMEs. The mass estimate is restricted to the width range $20^{\circ}$ to $120^{\circ}$ (inclusive). 


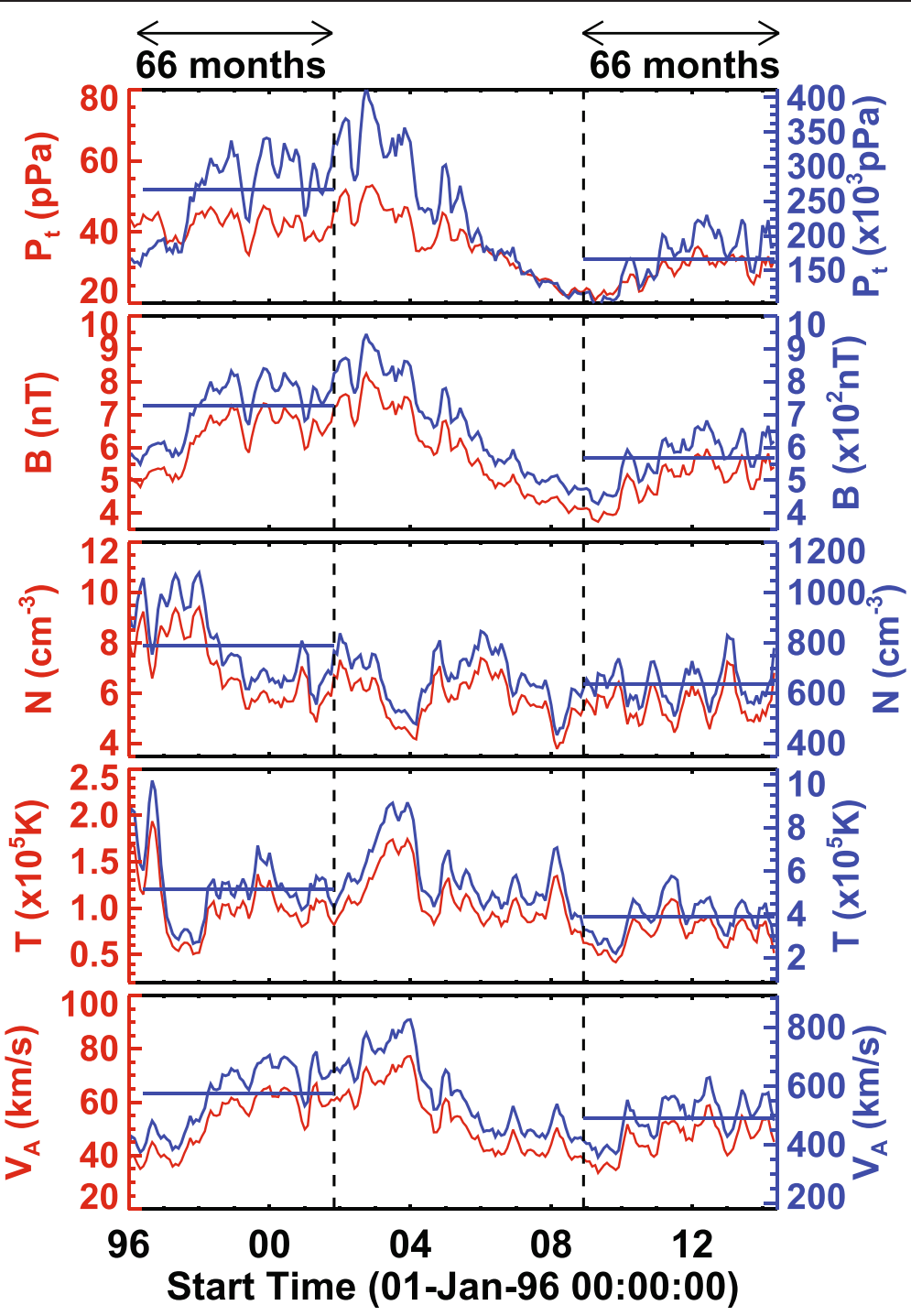

Figure 13 Physical parameters of the solar wind at 1 AU obtained from the OMNI database from January 1996 through May 2014: Total pressure $(\mathrm{Pt})$, magnetic field magnitude (B), proton density $(\mathrm{N})$, proton temperature (T), and the Alfvén speed (VA) at 1 AU (red lines with left-side Y-axis). The same quantities extrapolated from $1 \mathrm{AU}$ to the corona (20 Rs) are shown by the blue lines (right-side Y-axis), assuming that B, N, and T vary with the heliocentric distance R as R-2, R-2, and R-0.7, respectively. The blue bars denote the 66-month averages in each panel, showing the decrease of all the parameters in cycle 24.
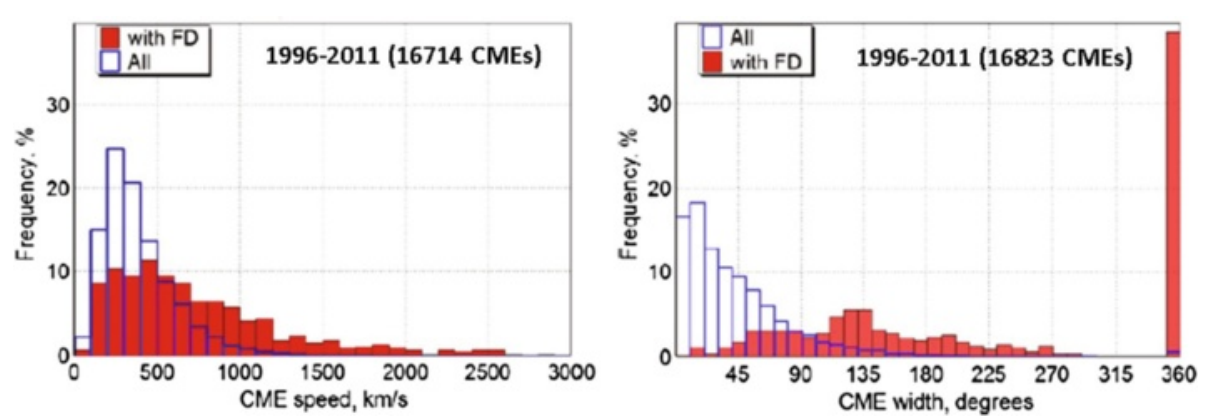

Figure 14 Comparison between the general population of CMEs (blue) and the FD-associated CMEs for CME speed (left) and width (right). All quantities were measured in the sky plane, and no projection correction has been made (from Belov et al. 2014). 
streams. So far, no 'blast wave' shocks have been detected in the interplanetary medium by spacecraft instrumentation. Shocks compress and heat the upstream plasma and magnetic fields (Kennel et al. 1985). Thus, the immediate downstream (or sheath) region may be visible at times. Shocks form from a steepening of magnetosonic waves. To identify whether a wave is a shock or not, it must be shown to have a supermagnetosonic speed in its normal direction. Methods of analyses can be found in Tsurutani and Lin (1985) and the geoeffectiveness of shocks and discontinuities in Tsurutani et al. (2011b).

There have been several recent studies on white-light shocks (Vourlidas et al. 2003; Michalek et al. 2007; Gopalswamy et al. 2008a; Gopalswamy et al. 2009; Gopalswamy et al. 2009b; Ontiveros and Vourlidas 2009; Bemporad and Mancuso 2011; Gopalswamy and Yashiro 2011; Maloney and Gallagher 2011; Kim et al. 2012; Poomvises et al. 2012) that have provided a better understanding of the CME structure beyond the classical three-part structure (Hundhausen 1987). The availability of STEREO and SDO observations increased our ability to visualize the CME-shock system and understand the shock formation and coronal plasma properties.

The dome structure surrounding newly erupted CMEs has been recognized as the three-dimensional counterpart of the so-called EIT waves (Patsourakos and Vourlidas 2009; Veronig et al. 2010; Ma et al. 2011; Kozarev et al. 2011; Warmuth and Mann 2011; Gallagher and Long 2011; Harra et al. 2011; Gopalswamy et al. 2012b; Selwa et al. 2013; Temmer et al. 2013; Liu and Ofman 2014; Nitta et al. 2013a). The wave nature of EUV waves was also established based on the fact that they are reflected from nearby coronal holes (Long et al. 2008, 2013; Gopalswamy et al. 2009c; Olmedo et al. 2012; Shen et al. 2013a; Kienreich et al. 2013: Kwon et al. 2013). Gopalswamy and Yashiro (2011) estimated the coronal magnetic field within the $\mathrm{SOHO}$ coronagraphic field of view (6 to $23 \mathrm{Rs}$ ) using the fact that the standoff distance of the white-light shock with respect to the radius of curvature of the driving flux rope is related to the shock Mach number and the adiabatic index (Russell and Mulligan 2002; Savani et al. 2012). Since the shock speed is measured from the coronagraphic images, these authors were able to derive the Alfvén speed and magnetic field in the ambient medium. Poomvises et al. (2012) extended this technique to the interplanetary medium and showed that the derived magnetic field strength is consistent with the HELIOS in situ observations. This technique will be extremely important to compare future in situ observations from missions to the Sun such as Solar Orbiter and Solar Probe Plus, currently under development (Müller et al. 2013). The standoff-distance technique was also applied to a CME-shock structure observed by SDO on 13 June 2010, which showed that the technique can work as close to the Sun as 1.20
Rs, where the shock first formed (Gopalswamy et al. 2012b; Downs et al. 2012). The shock formation heights derived from SDO/AIA and STEREO/EUVI have provided direct confirmation that CME-driven shocks have enough time to accelerate particles to $\mathrm{GeV}$ energies from a height of approximately 1.5 Rs before they are released when the CME reaches a height of about 3 to 4 Rs (Gopalswamy et al. 2013a,b; Thakur et al. 2014). The low shock formation height applies only to those CMEs, which quickly accelerate and attain high speeds (see e.g., Bein et al. 2011).

\section{Shocks inferred from radio observations}

Type II radio bursts in the metric domain, traditionally observed from ground-based observatories, indicate shock formation very close to the Sun (e.g., Kozarev et al. 2011; Ma et al. 2011; Gopalswamy et al. 2012b). Imaging these bursts provides important information such as the magnetic field in the ambient medium (Hariharan et al. 2014). These bursts indicate the height of shock formation in the corona as evidenced by EUV shocks and Moreton waves (see e.g., Asai et al. 2012a, 2012b). Radio emission from interplanetary shocks in the form of type II bursts provides important information of shock propagation in the heliosphere (Gopalswamy 2011). CMEs with continued acceleration beyond the coronagraph field of view (FOV) may form shocks at large distances where they become super-magnetosonic (faster than the upstream magnetosonic wave speed). Shocks forming at large distances of the Sun may or may not produce type II radio bursts (Gopalswamy et al. 2010c). Radio-quiet CMEs (those lacking type II radio bursts) typically have positive acceleration in the coronagraphic field of view and become super-magnetosonic in the interplanetary (IP) medium at large heliocentric distances. Deceleration of radio-loud CMEs near the Sun and the continued acceleration of radio-quiet CMEs into the IP medium make them appear similar at $1 \mathrm{AU}$. However, there is a better chance that radio-loud CMEs produce an energetic storm particle event (Mäkelä et al. 2011) and strong sudden commencement/sudden impulse (Veenadhari et al. 2012), suggesting that stronger shocks near the Sun do matter. In fact Vainio et al. (2014) have shown that the cutoff momentum of particles observed at $1 \mathrm{AU}$ can be used to infer properties of the foreshock and the resulting energetic storm particle event, when the shock is still near the Sun.

By combining STEREO/HI (heliospheric imager) observations, interplanetary radio bursts observations, and in situ measurements from multiple vantage points, Liu et al. (2013) showed that it is possible to track CMEs and shocks. In particular, they were able to study CME interaction signatures in the radio dynamic spectrum. The drift rate of the type II radio bursts can also be converted into 
shock speed for comparison with the CME speed derived from HI observations, providing a method to predict shock arrival (e.g., Xie et al. 2013a).

\section{Shock critical Mach numbers}

There is renewed interest in shock critical Mach numbers and their evolution with heliocentric distance (Gopalswamy et al. 2012b; Bemporad and Mancuso 2011, 2013; Vink and Yamazaki 2014). For example, some radio-loud shocks may dissipate before reaching 1 AU (Gopalswamy et al. 2012c) indicating that the Mach number is dropping to 1 or below. Bemporad and Mancuso (2011) concluded that the supercritical region occupies a larger surface of the shock early on but shrinks to the nose part of the shock as it travels away from the Sun. Vink and Yamazaki (2014) introduced a different critical Mach number (Macc), which is substantially larger than the first critical Mach number (Mcrit) of quasiparallel shocks (Kennel et al. 1985) but similar to Mcrit of quasi-perpendicular shocks. According to these authors, the condition Macc $>\sqrt{5}$ seems to be required for particle acceleration, which may be relaxed when seed particles exist.

The clear identification of an interplanetary magnetic cloud (MC) with a CME by Burlaga et al. (1982) replaced the Gold bottle by a flux rope. Although the MC definition by Burlaga et al. (1982) was narrower than the flux rope definition (magnetic field twisted around an axis), the terms 'flux rope' and 'MC' are interchangeably used after Goldstein (1983) showed that the MC magnetic field can be modeled by a flux rope. All theories of CME eruption and propagation use the flux rope as the fundamental structure in their calculations, either preexisting or formed during eruption (Yeh 1995; Chen et al. 1997; Riley et al. 2006; Forbes et al. 2006; Chen 2012; Kleimann 2012; Lionello et al. 2013; Janvier et al. 2013; Démoulin 2014 Extensive CME observations from the $\mathrm{SOHO}$ mission have helped perform many studies on CME flux ropes. Chen et al. (1997) showed that the observed CME structure in the LASCO field of view can be interpreted as the two-dimensional projection of a three-dimensional magnetic flux rope with its legs connected to the Sun.

The CME flux rope is thought to be either pre-existing or formed out of reconnection during the eruption process and is observed as an MC in the interplanetary medium (see e.g., Gosling 1990; Leamon et al. 2004; Qiu et al. 2007). On the other hand, it is possible that a set of loops from an active region on the Sun can simply expand into the interplanetary (IP) medium and can be detected as an enhancement in the magnetic field with respect to the ambient medium (Gosling 1990) without any flux-rope structure. The in situ magnetic signatures will be different in the two cases. A spacecraft passing through the flux rope center will see a large, smooth rotation of the magnetic field throughout the body of the interplanetary CME (ICME), while the expanded loop system will show no rotation. If we take just the IP observations, we may be able to explain MCs as flux ropes and non-MCs as expanding loops. However, they should show different charge-state characteristics (see e.g., Aguilar-Rodriguez et al. 2006; Gopalswamy et al. 2013c) because of the different solar origins. The flux rope forms during the flare process and hence is accessed by the hot plasma resulting in high charge states inside MCs when observed at 1 AU. Expanding loops, on the other hand, should not have high charge states because no reconnection is involved. Riley and Richardson (2013) analyzed Ulysses spacecraft measurements of ICMEs and concluded the ICME may not appear as MCs because of observing limitations or the initiation mechanism at the Sun may not produce MCs.

In a series of two coordinated data analysis workshops (CDAWs), a set of structure of CMEs, 54 CME-ICME pairs were analyzed to study the fluxrope nature of CMEs (see Gopalswamy et al. 2013d for the list of papers based on these CDAWs). It was found that MCs and non-MCs were indistinguishable based on their near-Sun manifestations such as white-light CMEs and flare post-eruption arcades. In particular, the CMEs were fast and the flare arcades were well defined (Yashiro et al. 2013). Fe and $\mathrm{O}$ charge states at $1 \mathrm{AU}$ were also indistinguishable between MCs and non-MCs, suggesting a similar eruption mechanism for both types at the Sun (Gopalswamy et al. 2013c). Combined with the fact that CMEs can be deflected toward or away from the SunEarth line (Gopalswamy et al., 2009d), the observing geometry (i.e., the observing spacecraft may not cross the flux rope axis) seems to be the primary reason for the non-MC appearance of flux ropes (see e.g., Kim et al. 2013). Many authors have advocated that all ICMEs are flux ropes (Marubashi 2000; Owens et al. 2005; Gopalswamy 2006a), but the single point observations at 1 AU may miss it. Marubashi et al. (2015) showed that almost all ICMEs can be fit to a flux rope if a locally toroidal flux rope model is considered in addition to the cylindrical flux rope model. Similarly, the active region helicity and the helicity of the ICMEs were in good agreement (Cho et al. 2013).

Using SDO/AIA data, Zhang et al. (2012b) reported that flux ropes exist as a hot channel before and during an eruption. The structure initially appeared as a twisted and writhed sigmoid with a temperature as high as $10 \mathrm{MK}$ and then transformed into a semi-circular structure during the slow-rise phase, which was followed by a fast acceleration and flare onset. Cheng et al. (2013) reported that the hot channel rises before the first appearance of the CME leading front and the flare onset of the associated flare. These 
results indicate that the hot channel acts as a continuous driver of the CME formation and eruption in the early acceleration phase. Li and Zhang (2013a, 2013b) reported on the eruption of two flux ropes from the same active region within 25 minutes of each other on 23 January 2012. The two flux ropes initially rose rapidly, slowed down, and accelerated again to become CMEs in the coronagraph FOV. The two CMEs were found to be interacting in the coronagraph FOV as observed by SOHO and STEREO (Joshi et al. 2013). Li and Zhang (2013c) also studied homologous flux ropes from active region 11745 during 20 to 22 May 2013. All flux ropes involved in the eruption had a similar morphology.

\section{Shock normal angles and particle acceleration}

It has been shown by detailed studies that the normal of the shocks relative to the upstream magnetic field is important for the efficiency of particle acceleration. The physical reasoning is that for quasi-parallel shocks where the normal and upstream field are nearly aligned, shockreflected particles create upstream waves by beaming instabilities (Tsurutani and Rodriguez, 1981; Tsurutani et al. 1983b). The upstream (and downstream) waves act as two walls of a Fermi accelerator (Lee, 1983) leading to exceptional particle acceleration. Evidence of this has been demonstrated by Kennel et al. 1984a, 1984b). This microscale structure was not examined in the particle acceleration studies discussed in earlier sections.

\section{Propagation effects: deflection, interaction, and rotation of CMEs}

Once a CME is ejected from the Sun, its 3D geometry at a far-away location such as Earth depends on the evolution in the changing background solar wind and magnetic field (Temmer et al. 2011). The CME flux ropes expand, so the magnetic content typically decreases. CMEs can be deflected in the latitudinal and longitudinal directions by pressure gradients (magnetic + plasma). The CME flux ropes can also be distorted by changing flow speeds in the background. Finally, CMEs may also rotate, so the orientation of the magnetic field inferred from solar observations may not match what is observed at Earth. It is possible that many of these effects can occur simultaneously (NievesChinchilla et al. 2012). At present, there are a few techniques to connect CMEs observed at the Sun with their interplanetary counterparts. Interplanetary type II bursts detected by Wind/WAVES and STEREO/WAVES instruments can track CME-driven shocks all the way from the Sun to the observing spacecraft located at $1 \mathrm{AU}$ (Xie et al. 2013a). Interplanetary scintillation (IPS) observations track turbulence regions surrounding CMEs (typically the sheath region) also over the Sun-Earth distance (see e.g., Manoharan 2010; Jackson et al. 2013). The heliospheric imagers on board STEREO track CMEs in white light over the Sun-Earth distance (e.g. Möstl and Davies 2013).

A combination of CME tracking in the inner heliosphere using STEREO heliospheric imagers (HIs) and numerical simulations have greatly enhanced CME propagation studies. The interaction of CMEs with the ambient medium is the primary propagation effect. This interaction is represented by an aerodynamic drag that dominates beyond the coronagraphic field of view. Close to the Sun, the propelling force and gravity dominate (see e.g., Vrsnak and Gopalswamy 2002). Defining the background is one of the key inputs needed for understanding CME propagation (see e.g., Roussev et al. 2012; Arge et al. 2013). However, there are other processes that can significantly affect the propagation of CMEs: CME-CME interaction (see e.g., Gopalswamy et al. 2001a; 2012c; Temmer et al. 2012; Harrison et al. 2012; Lugaz et al. 2012; Liu et al. 2014a; Sterling et al. 2014; Temmer et al. 2014) and CME deflection by largescale structures such as coronal holes and streamers (Gopalswamy 2010, 2009d; Shen et al. 2011; Gui et al. 2011; Wood et al. 2012; Kay et al. 2013; Panasenco et al. 2013; Gopalswamy and Mäkelä 2014).

Different types of interaction become predominant during different phases of the solar cycle. During the rise phase, when polar coronal holes are strong and CMEs originate at relatively higher latitudes, the polar coronal holes are effective in deflecting CMEs (e.g., Gopalswamy et al. 2008b). During the maximum phase, CMEs occur in great numbers, so CME-CME interaction is highly likely (e.g., Gopalswamy et al. 2012c; Lugaz et al. 2013; Chatterjee and Fan 2013; Farrugia et al. 2013; Kahler and Vourlidas 2014). CME interactions can also result in CME deflection and merger (Shen et al. 2012). In the declining phase, low-latitude coronal holes appear frequently, so CME deflection by such coronal holes becomes important (Gopalswamy et al. 2009d; Mohamed et al. 2012; Mäkelä et al. 2013). The deflections are thought to be caused by the magnetic pressure gradient between the eruption region and the coronal hole (Gopalswamy et al. 2010d; Shen et al. 2011; Gui et al. 2011).

Determining the initial orientation of flux ropes has been possible by fitting a flux rope to coronagraph observations (Thernisien 2011; Xie et al. 2013b). Such fitting already provides a lot of information on the deviation of CME propagation direction from the radial (e.g., Gopalswamy et al. 2014b). Isavnin et al. (2014) defined such initial flux rope orientation using extremeultraviolet observations of post-eruption arcades and/or eruptive prominences and coronagraph observations. Then, they propagated the flux rope to $1 \mathrm{AU}$ in a MHDsimulated background solar wind and used in situ observations to check the results at $1 \mathrm{AU}$. They confirmed that the flux-rope deflection occurs predominantly 
within 30 Rs, but a significant amount of deflection and rotation happens between $30 \mathrm{Rs}$ and 1 AU. They also found that that slow flux ropes tend to align with the streams of slow solar wind in the inner heliosphere.

\section{CME arrival at Earth}

There have been many attempts recently to convert the knowledge gained on CME propagation to predict the arrival times at $1 \mathrm{AU}$. The CME travel time essentially depends on the accurate estimate of the space speed of CMEs and the background solar wind speed; these have been estimated based on single view (SOHO) as well as from multiple views (SOHO and STEREO). Davis et al. (2010) found that CME speeds derived from STEREO/ COR2 and Thernisien (2011) forward-fitting model were in good agreement, although CME speeds changed in the HI FOV depending on the near-Sun speed. Millward et al. (2013) developed the CME analysis tool (CAT), which models CMEs to have a lemniscate shape, which is similar to ice cream cone model. They showed that the leading-edge height and half-angular width of CMEs can be determined more accurately using multi-view data. Colaninno et al. (2013) tracked nine CMEs continuously from the Sun to near Earth in SOHO and STEREO images and found that the time of arrival was within \pm 13 h. Gopalswamy et al. (2013e) considered a set of 20 Earth-directed halos viewed by $\mathrm{SOHO}$ and STEREO in quadrature, so as to obtain the true earthward speed of CMEs. When the speeds were input to the empirical shock arrival (ESA) model, they found that the ESA model predicts the CME travel time within about $7.3 \mathrm{~h}$, which is similar to the predictions by the ENLIL model. They also found that CME-CME and CME-coronal hole interaction can lead to large deviations from model predictions. Vrsnak et al. (2014) compared the arrival-time predictions from the 'WSA-ENLIL + cone model' and the analytical 'drag-based model' (Vršnak et al. 2013) and found that the difference in predictions had an absolute average of $7.1 \mathrm{~h}$. Compared with observations, the drag-based model had an average absolute difference of $14.8 \mathrm{~h}$, similar to that for the ENLIL model (14.1 h). Xie et al. (2013a) compared travel times of CMEs when ENLIL + cone model and ENLIL + flux rope model were used. They found that the ENLIL + flux rope model results showed a slight improvement ( $4.9 \mathrm{~h}$ vs. $5.5 \mathrm{~h}$ ). They also found that predictions based on kilometric type II bursts improved significantly when the ENLIL model density was used rather than the average solar wind plasma density in deriving shock speeds from the type II drift rate. The improvement was typically better by approximately $2 \mathrm{~h}$. Möstl et al. (2014) derived the absolute difference between predicted and observed ICME arrival times for 22 CMEs as $8.1 \mathrm{~h}$ (rms value of $10.9 \mathrm{~h}$ ). Empirical corrections to the predictions reduced arrival times to within $6.1 \mathrm{~h}$ (rms value of $7.9 \mathrm{~h}$ ). Echer et al. (2010) attempted to identify the solar origins of the November 2004 superstorms on Earth using existing interplanetary propagation routines published in the literature. They found that during highly active solar intervals, the predictions were sometimes ambiguous, in agreement with the comments above. Thus, there has been a steady progress in predicting the arrival time of CMEs, which need to be continued and expended to the prediction of $\mathrm{Bz}$ values, which are crucial to predict the strength of geomagnetic storms.

\section{CMEs and geomagnetic storms}

One of the direct consequences of CMEs arriving at Earth's magnetosphere is the geomagnetic storm. The primary link between a geomagnetic storm and a CME is the out of the ecliptic component $(\mathrm{Bz})$ of the interplanetary magnetic field (Gonzalez et al., 1994; Zhang et al. 2007; Gopalswamy 2008; Echer et al. 2008a, 2008b, 2013; Cid et al. 2012). Echer et al. (2008a) conclusively showed that for all 90 major (Dst $<-100 \mathrm{nT}$ ) storms that occurred during cycle 23, it was the $\mathrm{Bz}$ component that was responsible for the storms (some people have thought that it was possible that the IMF By component was also important). When $\mathrm{Bz}$ is negative (south pointing), then the CME field reconnects with Earth's magnetic field (Dungey 1961) causing the geomagnetic storm. While the $\mathrm{Bz}$ component is negligible in the quiet solar wind, CMEs contain $\mathrm{Bz}$ by virtue of their flux rope structure. Fast CMEs drive shocks, so the compressed sheath field between the flux rope and the shock can also contain Bz (Tsurutani et al. 1988). Thus, both the flux rope and sheath can be the source of $\mathrm{Bz}$ and hence cause geomagnetic storms. One of the common indicators of the strength of geomagnetic storms is the Dst index (expressed in nT), which is computed as the horizontal component of Earth's magnetic field measured at several equatorial stations (now, a SYM-H index is available which is essentially a 1-min resolution Dst index). Yakovchouk et al. (2012) reported significant difference between the local and global peak storm intensities: the local storm minima were found to be $25 \%$ to $30 \%$ stronger than the global minima. Here, we consider only global peak intensities. Major storms have Dst $\leq-100 \mathrm{nT}$ and are mostly caused by CMEs. Echer et al. (2008a) studied all 90 storms with Dst $\leq-100 \mathrm{nT}$ for cycle 23. They divided ICMEs proper from their upstream sheaths. They found that roughly half of the storms that were caused by $\mathrm{CME} /$ sheaths were due to CMEs and half due to sheath fields. Yakovchouk et al. (2012) found that $10 \%$ of major storms are caused by CIRs. Echer et al. (2008a) determined that $13 \%$ of the cycle 23 storms were caused by CIRs.

It should be noted that all studies of 'superstorms' or storms with Dst intensities $<-250 \mathrm{nT}$ have been caused by magnetic clouds (Tsurutani et al. 1992a; Echer et al. 2008b). Such intense storms are not caused by sheaths 
or CIRs. One might ask why not? The argument is a simple one. The slow solar wind magnetic field is approximately 5 to $7 \mathrm{nT}$. It has been shown by Kennel et al. (1985) that fast shocks can compress the magnetic field by a maximum factor of approximately 4, regardless of the shock Mach number. Thus, interplanetary sheath fields and CIR magnetic fields should have maximum field strengths of approximately 20 to $35 \mathrm{nT}$. In contrast, magnetic cloud fields have been 50 to $60 \mathrm{nT}$ and in exceptional cases approximately $100 \mathrm{nT}$. Thus, even if the sheath and CIR magnetic fields are totally southward, they are small by comparison to MC fields.

There is one exception to this above explanation. In cases of ARs where there are multiple CMEs and multiple shocks, the shocks can 'pump up' sheath magnetic field intensities. This has been shown to be the case for both CAWSES intervals of study (Tsurutani et al. 2008; Echer et al. 2011c.

One of the early signatures of the weak solar cycle 24 has been the drastically reduced number of major (Dst $\leq-100 \mathrm{nT}$ ) geomagnetic storms (Echer et al. 2011b; 2012; Gopalswamy 2012; Richardson 2013; Kilpua et al. 2014). A plot of the Dst index as a function of time in Figure 15 shows that the frequency and amplitude of the storms in cycle 24 are the lowest in the space age (cycles 19 to 24). There were storms with Dst < -200 nT in every cycle since 1957, except in cycle 24, in which the storms never exceeded a strength of $140 \mathrm{nT}$. Several historical storms (including the recent ones on 14 March 1989 with Dst $=-589 \mathrm{nT}$ and on 20 November 2003 with

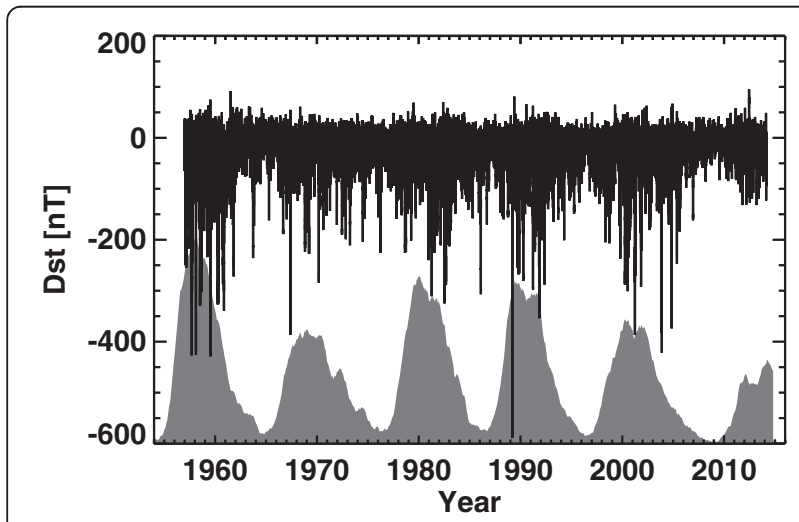

Figure 15 A plot of the Dst index since 1957 from the World Data Center, Kyoto (http://wdc.kugi.kyoto-u.ac.jp/dstdir/). The vertical streaks extending beyond $-100 \mathrm{nT}$ are the major storms. The largest storm (Dst approximately -589 nT) is the one on 13 March 1989 storm, which caused the power blackout in Quebec, Canada (Allen et al. 1989). The Halloween 2003 storm was the next largest storm (Dst $<-400 \mathrm{nT}$ ) after the Quebec storm (Mannucci et al. 2005). The positive excursions are the sudden commencements or sudden impulses caused by the impact of CME-driven shocks on the magnetosphere. The sunspot number is plotted at the bottom for reference.

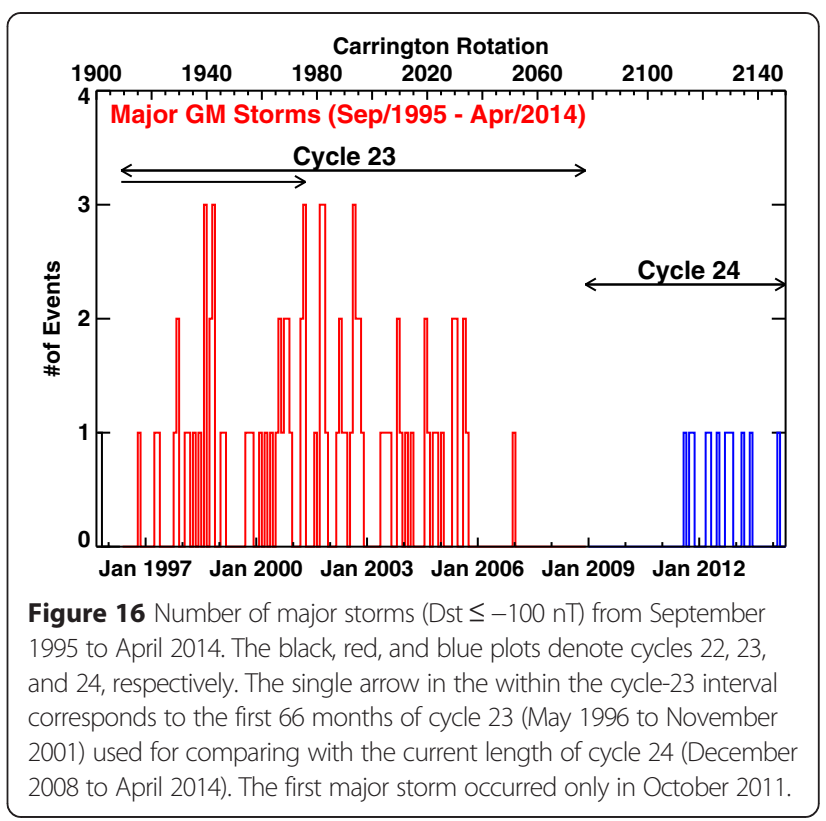

Dst $=-422 \mathrm{nT}$ ) can be found in the plot (see Cliver et al. 1990; Gopalswamy et al. 2005b,c).

Figure 16 compares the number of major storms as a function of time for cycles 23 and 24, including the last couple of events of cycle 22. We see that the gap between the last storm of cycle 22 and the first storm of cycle 23 was only approximately 16 months. On the other hand, the gap was about four times larger between cycles 23 and 24 as the Sun emerged out of the deep solar minimum following cycle 23 . The number of storms in the corresponding phases of cycles 23 and 24 were 37 and 11 , respectively, indicating a $70 \%$ reduction of major storms in cycle 24 . This is considerably more than the reduction of the number of fast and wide CMEs from the disk center or the overall reduction in the number of energetic CMEs $(<30 \%$, see Table 1$)$. Gopalswamy et al. (2014a) showed that the reduction in the heliospheric total pressure (plasma + magnetic) makes CMEs expand more in cycle 24 thereby reducing their density and magnetic content that ultimately result in weaker storms. This can be understood from the empirical relation between the Dst and the product $\mathrm{VBz}$ of the interplanetary structure causing the storm: Dst $=-0.01 \mathrm{VBz}-32 \mathrm{nT}$ (Wu and Lepping 2002; Gopalswamy 2010).

Table 2 shows the list of major storms from cycle 24 (updated to the end of 30 June 2014 from Gopalswamy 2012). There were only 12 major storms with the Dst ranging from -103 to $-137 \mathrm{nT}$. Two storms with the Dst close to $-100 \mathrm{nT}$ are also included in Table 2. All storms were due to CMEs, except one caused by a CIR (1 June 2013). The average Dst of the $11 \mathrm{CME}$ storms was only -119 nT. Over the corresponding epoch in cycle 23, there were 36 storms with an average Dst of $-158 \mathrm{nT}$. 
Table 2 Major geomagnetic storms of cycle 24 (Dst <-100 nT)

\begin{tabular}{|c|c|c|c|c|c|c|c|}
\hline Number & Date and time of storm & Dst (nT) & CME onset & $\mathrm{V}^{\mathrm{a}} \mathrm{km} / \mathrm{s}$ & $W^{b}$ degree & Eruption location & Bz (nT) location ${ }^{c}$ \\
\hline 1 & $2011080604: 00$ & -107 & 08/04 04:12 & 1,315 & $\mathrm{H}$ & N19W36 & $-21.4 \mathrm{Sh}$ \\
\hline 2 & $2011092700: 00$ & -103 & 09/24 12:48 & 1,915 & $\mathrm{H}$ & N15E58 & $-28.7 \mathrm{Sh}$ \\
\hline 3 & $2011102502: 00$ & -137 & 10/22 01:25 & 593 & $\mathrm{H}$ & $\mathrm{N} 40 \mathrm{~W} 30^{\mathrm{d}}$ & $-19.9 \mathrm{Sh}$ \\
\hline 4 & 20120309 09:00 & -133 & 03/07 01:36 & 1,825 & $\mathrm{H}$ & N17E27 & -17.7 FS \\
\hline 5 & 20120424 05:00 & -107 & 04/19 15:12 & 540 & 142 & S30E $71^{d}$ & $-15.3 \mathrm{FS}$ \\
\hline 6 & $2012071518: 00$ & -125 & 07/12 16:48 & 885 & $\mathrm{H}$ & S15W01 & -18.6 FS \\
\hline 7 & 20121001 04:00 & -133 & 09/28 00:12 & 947 & $\mathrm{H}$ & N06W34 & $-20.0 \mathrm{Sh}$ \\
\hline 8 & $2012100909: 00$ & -111 & 10/05 02:48 & 612 & 284 & $\mathrm{~S} 23 \mathrm{~W} 31^{\mathrm{d}}$ & $-16.2 \mathrm{FS}$ \\
\hline 9 & $2012111408: 00$ & -108 & $11 / 0915: 12$ & 559 & 276 & $\mathrm{~S} 18 \mathrm{E} 16^{\mathrm{d}}$ & -18.9 NS \\
\hline 10 & $2013031721: 00$ & -132 & 03/15 07:12 & 1,063 & $\mathrm{H}$ & $\mathrm{N} 11 \mathrm{E} 12$ & $-11.1 \mathrm{Sh}$ \\
\hline 11 & $2013060109: 00$ & -119 & $\mathrm{CIR}$ & - - & - - & $\mathrm{CH}$ & $-21.4 \mathrm{IR}$ \\
\hline 12 & 20140219 09:00 & -112 & 02/16 10:00 & 634 & $\mathrm{H}$ & S11E01 & $-15.5 \mathrm{FS}^{\mathrm{e}}$ \\
\hline
\end{tabular}

${ }^{a} \mathrm{CME}$ speed with an average value of $1,021 \mathrm{~km} / \mathrm{s} .{ }^{\mathrm{b}} \mathrm{CME}$ width $(\mathrm{H}=$ full halos: $7 / 11$ or $63 \%)$. ${ }^{\mathrm{C}}$ Origin of negative Bz: Sh, sheath; $\mathrm{FS}$, fully south (axis of the high inclination cloud pointing south); NS, north south cloud (negative Bz in the rear end of the cloud); IR, interface region between fast and slow winds. ${ }^{\mathrm{d} D i s a p p e a r i n g}$ solar filament (DSF) events with the centroid of the filament given. ${ }^{\text {Ta }}$ The sheath is a preceding FS MC.

Thus, the number of major storms in cycle 23 was at least three times more and 33\% stronger. The average speed of the CMEs in Table 2 is $1,025 \mathrm{~km} / \mathrm{s}$, compared to $722 \mathrm{~km} / \mathrm{s}$ for the corresponding epoch in cycle 23. i.e., cycle-24 CMEs producing major storms were faster than those in cycle 23 by $42 \%$. The halo fraction was comparable between the two cycles ( 7 out of 11 or $64 \%$ in cycle 24 compared to 19 out of 31 or $61 \%$ in cycle 23). In other words, cycle-24 CMEs need be faster to produce storms similar to the ones in cycle 23.

Six of the $11 \mathrm{CME}$ storms in Table 2 were due to southward $\mathrm{Bz}$ in the ICME sheaths. These include one event in which the sheath was actually a preceding magnetic cloud: the shock from a disk-center CME on 16 Feb 2014 from S11E01 associated with a M1.1 flare at 09:20 UT entered into a preceding fully south (FS) cloud. The source of the magnetic cloud itself is not clear, but most likely a faint CME associated with an eruption in the northwest quadrant on 14 Feb 2014 around 02:33 UT (a faint CME at 4:28 UT CME was barely discernible in LASCO images). The shock compressed the preceding $\mathrm{CME}$ and enhanced the $\mathrm{Bz}$ that caused the storm. This is a good example that ICMEs can be affected by shocks from other eruptions. In the remaining five storms, southward $\mathrm{Bz}$ was in the cloud portion. Even cycles such as cycle 24 are supposed to have more north-south (NS) clouds (the ones with leading northward $\mathrm{Bz}$ ). However, there was only one such cloud in Table 2. All others were of FS clouds, which are high-inclination clouds with south-pointing axial field. Note that the $\mathrm{Bz}$ values ranged from -11.1 to $-28.7 \mathrm{nT}$ with an average of $-18.5 \mathrm{nT}$. The $\mathrm{Bz}$ values in cycle 24 were in a narrower range compared to those in cycle 23.

\section{Flares and the ionosphere}

It has long been known that solar flares create sudden ionospheric disturbances or SIDs (Thome and Wagner 1971; Mitra 1974). However, the extreme intensity of the Halloween flares and the rise of using global positioning systems (GPS) for ionospheric research has allowed major advances to be made in flare ionospheric research (Tsurutani et al. 2005; Afraimovich et al. 2009). Now with ground-based receivers virtually everywhere on Earth (with ocean coverage still a bit of a problem), high-time resolution, global coverage is now possible.

Figure 17 shows the change in the ionospheric total electron content (TEC) during the peak of the 28 October 2003 solar flare, from 11:00 to 11:08 UT. The flare peak time was taken from the unsaturated SOHO SEM narrow band EUV detector. The NOAA GOES X-ray detector was saturated. Thomson et al. (2004) has estimated its strength of the flare as large as $\mathrm{X} 45 \pm 5$ via other means. A quiet day background of 27 October was subtracted from the 28 October data to get this difference plot in Figure 17. The subsolar point (Africa) is at the center of the figure. The data points are individual ground observations of GPS satellites.

The solar flare causes the largest TEC enhancement at the subsolar region with a TEC enhancement of 22 TEC units. The nightside region shows no TEC change, as expected. This is the largest ionospheric TEC change due to a solar flare ever detected.

Figure 18 shows the simultaneous onset of the 28 October flare (both at SOHO and GOES), the Libreville ionospheric TEC enhancement and the dayglow enhancement at approximately 11:00 UT. The SEM data (unsaturated) shows a double peak structure. The ionospheric TEC rose from approximately 


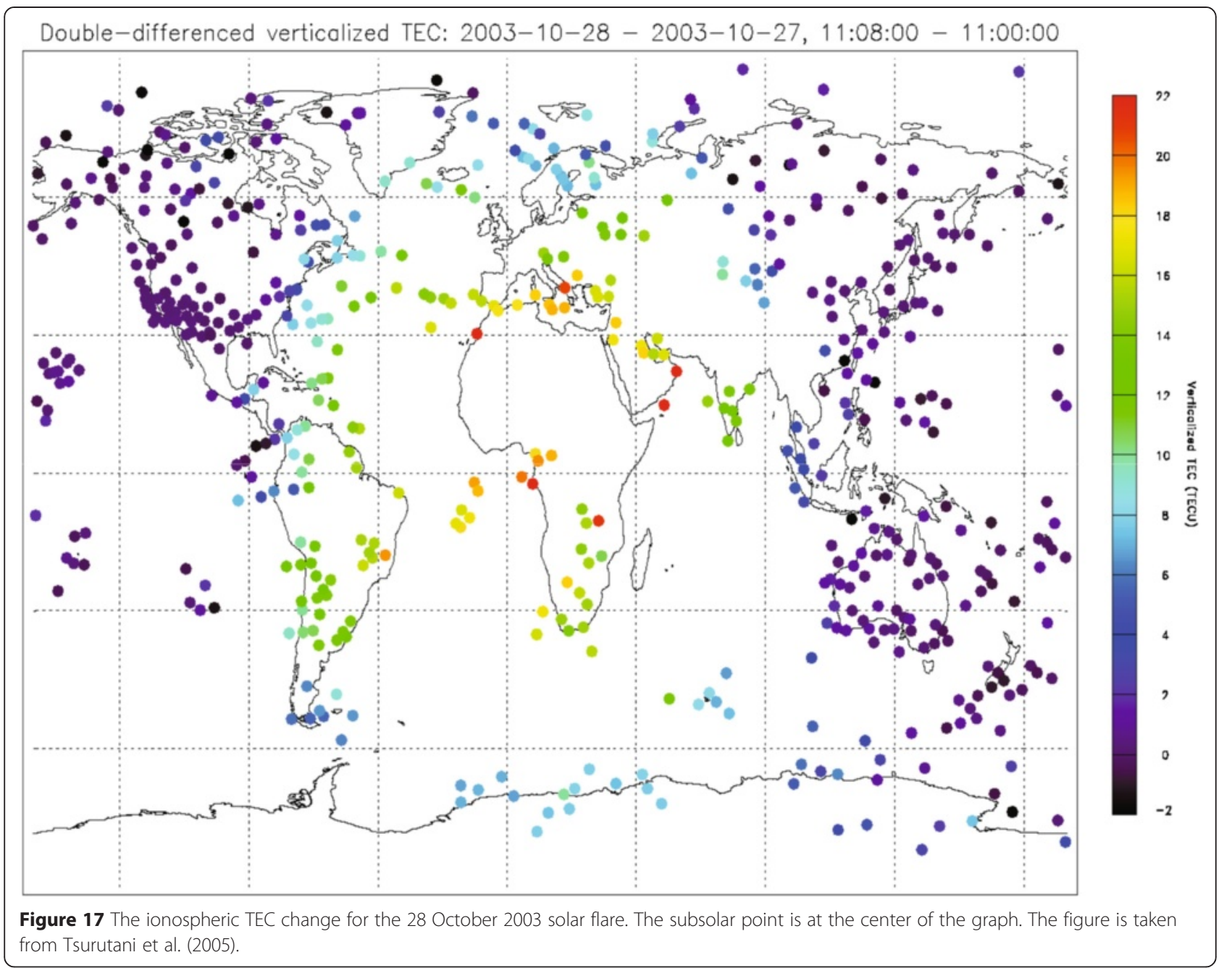

11:00 UT to approximately 11:05 UT and then less rapidly from 11:05 to approximately 11:18 UT, where a peak value of approximately 25 TEC above background was attained. One hour prior to the flare, the background TEC was 82 TECU so the flare caused an approximately $30 \%$ increase in the ionospheric content in this region. This is the largest flare-TEC event on record. The dayglow (from the TIMED GUVI O 135.6 $\mathrm{nm}$ and N2 LBH 141.0- to 152.8-nm band) increased most rapidly from approximately 11:00 to approximately 11:04 and peaked at approximately 11:15 UT. The TEC enhancement lasted far longer (approximately $3 \mathrm{~h}$ ) than the flare itself (approximately 20 min). The cause is that the EUV portion of the flare causes photoionization at altitudes above approximately $170 \mathrm{~km}$ where the recombination time scale is hours (Tsurutani et al. 2005).

\section{CMEs and the ionosphere}

The southward magnetic component of ICMEs (and their upstream sheaths) creates magnetic storms, which are enhancements in the Earth's outer radiation belts. The magnitude is measured by ground-based magnetometers near the equator giving the Dst and SYM-H indices. CMEs/magnetic storms also cause severe ionospheric effects as well. Energetic particle precipitation into the auroral zones lead to local heating and neutral atmospheric expansion called the 'disturbance dynamo' (Blanc and Richmond 1980; Scherliess and Fejer 1997). It has also been noted that the interplanetary electric field reaches ionospheric levels (Nishida 1968; Kelley et al. 2003) causing other effects. More recently, it was noted that CME interplanetary electric fields penetrated down to the equatorial ionosphere and lasted for hours (Tsurutani et al. 2004). The electric fields were 'unshielded', contrary to theoretical expectations. The unshielded storm-time electric fields lead to what is called the 'daytime superfountain effect' illustrated in Figure 19.

Figure 19 shows the 'dayside superfountain effect' for the 30 October 2003 Halloween magnetic storm. The CHAMP satellite passes before the storm (blue trace), which shows the two equatorial ionization anomalies 

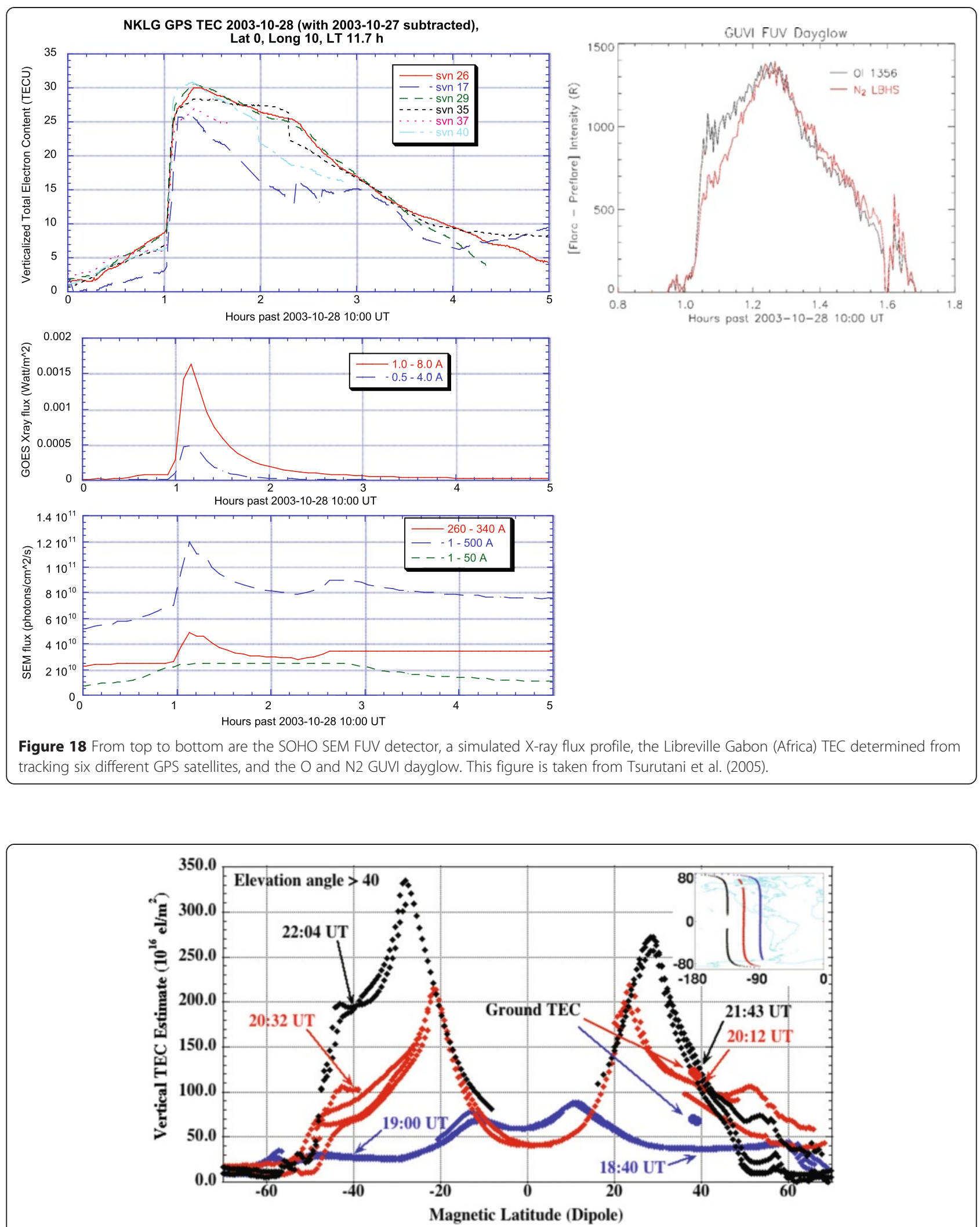

Figure 19 The vertical TEC above the CHAMP satellite (approximately 400-km altitude) for the 30 October 2003 magnetic storm. The blue curve is the TEC prior to the magnetic storm. The red and black traces are the overhead TEC values after storm onset. The figure is taken from Mannucci et al. (2005). 
(EIAs) located at approximately $\pm 10^{\circ}$. With time, the ionosphere and EIAs are uplifted to higher magnetic latitudes and have higher intensities. In the first pass after storm onset (red curve), the EIAs have peak intensities of approximately 200 TECU at approximately $\pm 20^{\circ}$ MLAT. In the following pass, a peak intensity of approximately $330 \mathrm{TECU}$ is detected at approximately $30^{\circ}$ MLAT. The cause of this remarkable feature is the interplanetary dawn-dusk electric field which uplifts the upper ionosphere by $\mathrm{E} \times \mathrm{B}$ convection (the Earth's magnetic field is aligned in a north-south direction at the magnetic equator). As the electrons and ions are convected to higher altitudes and latitudes, solar irradiation replaces the uplifted plasma by photoionization, leading to an overall increase in the TEC (Tsurutani et al. 2004; Mannucci et al. 2005).

\section{Coronal holes and CIRs}

Coronal holes play a number of important roles in SunEarth connection. Polar coronal holes indicate the strength of the polar field and hence the level of solar activity in the following cycle (Gopalswamy et al. 2012a; Selhorst et al. 2011; Shibasaki 2013; Mordvinov and Yazev 2014; Altrock 2014). Coronal holes in the equatorial region are good indicators of imminent high-speed streams (HSS) and CIRs arriving at Earth (Tsurutani et al. 1995, 2006; Cranmer 2009; Verbanac et al. 2011; Akiyama et al. 2013; Borovsky and Denton 2013). The empirical relationships established between HSS characteristics and the related geomagnetic activity provides an advance warning of impending CIR storms (Tsurutani et al. 2006; Verbanac et al. 2011). Coronal holes also deflect CME-driven shocks and CMEs that have important space weather consequences (Gopalswamy 2010, 2009d; Olmedo et al. 2012; Kay et al. 2013; Mäkelä et al. 2013). The deflection by coronal holes can be so large that CMEs originating from close the disk center of the Sun do not arrive at Earth while the shocks do. Coronal-hole deflection may also make the shock and the driving flux rope appear unaligned (Wood et al. 2012). Both high-speed streams and CIRs result in various types of magnetospheric responses (Tsurutani et al. 2006; Denton and Borovsky 2012; Borovsky and Denton 2013). Coronal holes also seem to play a critical role in deciding whether CMEs originating at latitudes $>30^{\circ}$ can produce groundlevel enhancement events (Gopalswamy and Mäkelä 2014), although their effect on large SEP events has not been conclusive (Kahler et al. 2014). In this section, we provide an overview of the recent progress on the geomagnetic response.

\section{CIRs, CIR storms, and HSS geomagnetic activity}

High-speed streams (HSSs) originate from coronal holes and form CIRs when they collide with the slower solar wind ahead (see e.g., Smith and Wolf 1976; Gosling 1996). The compressed interaction region has a higher density and temperature, and the magnetic field intensities and fluctuations are amplified. When the field within the CIR contains southward $\mathrm{Bz}$, geomagnetic activity ensues (Borovsky 2013). However, the fields within CIRs are typically highly fluctuating (compressed Alfvén waves). Thus, the character is considerably different from that of the magnetic fields within magnetic clouds. CIRs typically do not cause magnetic storms with Dst $<-100$ nT (Tsurutani et al. 1995, 2006; Echer et al. 2008a). The geomagnetic activity is typically in the range $-50 \mathrm{nT}<$ Dst $<-100 \mathrm{nT}$. Geomagnetic storms caused by CMEs and CIRs (plus the following HSSs) differ in some important ways (Denton et al. 2006; Jordanova et al. 2012; Liemohn and Katus 2012; Verbanac et al. 2013; Borovsky and Denton 2013; Keesee et al. 2014). For example, the CMEs and CIRs (plus HSSs) have different magnetospheric responses resulting in different development of various current systems and geomagnetic activity within the Earth's magnetosphere and ionosphere (Mannucci et al. 2005, 2008, 2012; Thayer et al. 2008; Lei et al. 2008, 2011; Verkhoglyadova et al. 2011, 2013, 2014; Verbanac et al. 2013). Keesee et al. (2014) performed superposed epoch analysis of 21 CME-driven and 15 CIR-driven storms during the June 2008 to April 2012 time frame and different evolutions of the ion temperature: the ion temperature increased in the recovery phase of CIR storms, while it increased rapidly at the onset of CME storms and cooled off during the main phase. Borovsky and Denton (2013) compared CIR storms associated with helmet streamers and pseudo-streamers. They found that pseudo-streamer CIR storms tend not to have a calm (Tsurutani et al. 1995) before the storm, with weaker superdense plasma sheet and electron radiation belt dropout.

\section{Effect of the weak solar activity}

Denton and Borovsky (2012) compared the magnetospheric effects of 93 strong (maximum speed approximately $600 \mathrm{~km} / \mathrm{s}$ ) and 22 weak (maximum speed approximately $500 \mathrm{~km} / \mathrm{s}$ ) high-speed streams. The weak HSSs were observed during the extended minimum that followed cycle 23. The strong HSSs were from earlier periods. A superposed epoch analysis showed that the solar wind velocity, in combination with the southward component of the IMF, largely governs the magnetospheric response to HSSs. In particular, the ring current was stronger and the magnetospheric electron flux was higher in the strong HSSs (see Figure 20). They point out that the difference in the evolution of particle flux can be attributed to the physical conditions in the magnetosphere that differ significantly under the two types of HSSs. 


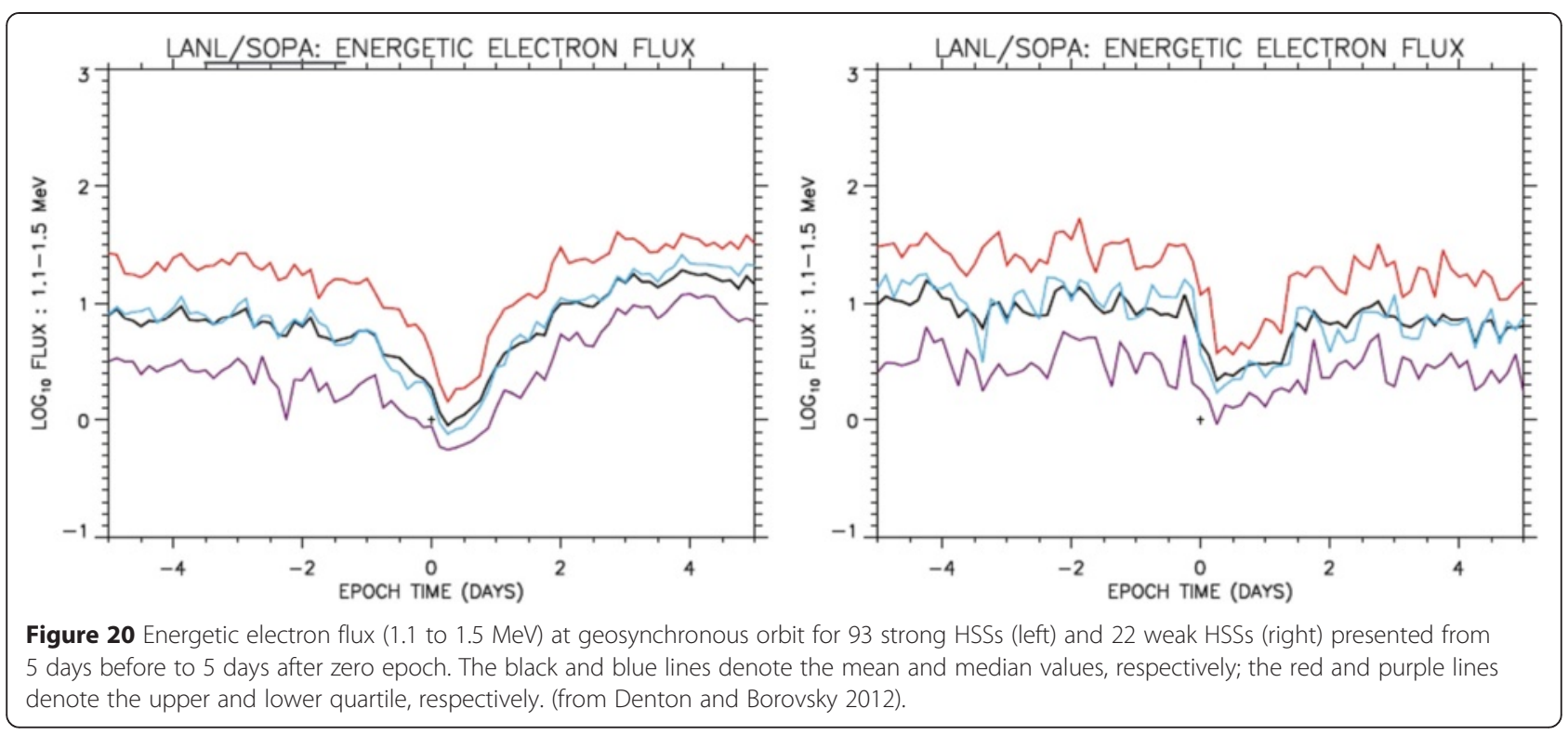

Even though the CIRs were weak during the prolonged minimum (see e.g., Echer et al. 2011a), they had interesting effects on the ionosphere and atmosphere. The ionospheric response to the weak CIRs was marginal but observable (Araujo-Pradere et al. 2011). However, the weak recurrent geomagnetic activity due to CIRs did produce distinct variability in the thermospheric density at an altitude of $400 \mathrm{~km}$ above ground (Lei et al. 2011 and references therein). The thermosphere was found to respond globally with the density varying by approximately $75 \%$. Most importantly, they were able to isolate the effect of geomagnetic activity from the EUV forcing because the EUV flux remained roughly constant during these CIR intervals. Thermospheric density variations also showed the periodicities in CIRs due to the spatial distribution of low-latitude coronal holes on the Sun, as did the ionosphere.

Hajra et al. (2013) studied a subset of high-speed streams, those that had particularly high geomagnetic activity associated with them, called High-Intensity Long-Duration Continuous Geomagnetic Activity (HILDCAA). These events are defined by occurring outside magnetic storms (thus in the HSS proper), lasting at least 2 days and having a peak $\mathrm{AE}>1,000 \mathrm{nT}$. The AE could not drop below $200 \mathrm{nT}$ for longer than 2 h. Hajra et al. (2013) found that the HILDCAAs ordered high-speed streams quite well. The solar

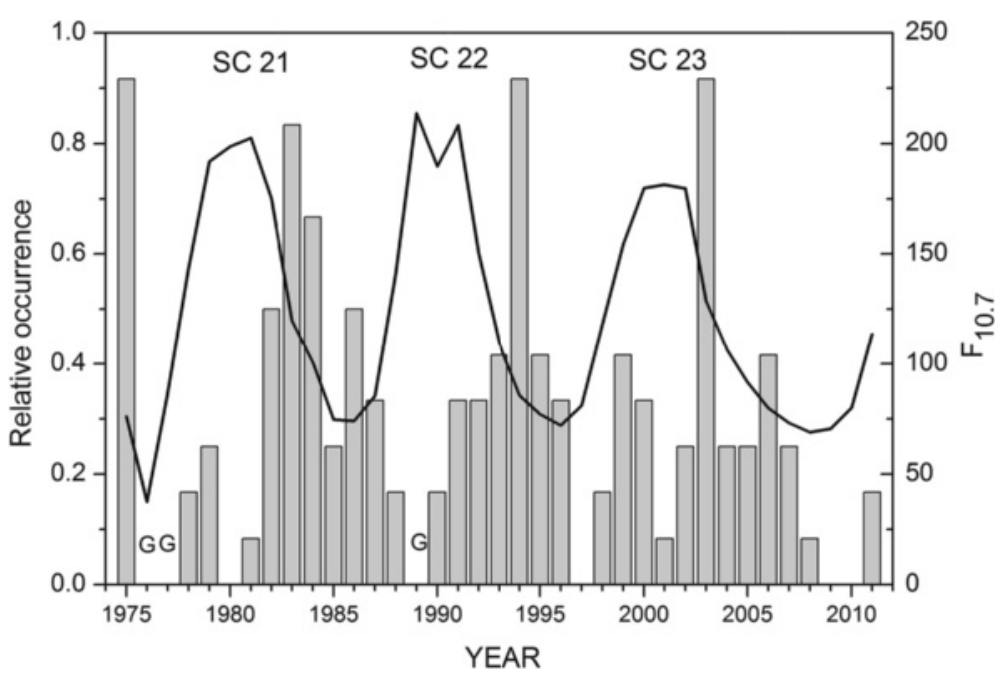

Figure 21 The solar cycle dependence of HILDCAAs. The relative occurrence of HILDCAAs during cycles 21, 22, and 23. The F10.7 solar flux is given as a black line. 'G' represents data gaps. No AE data were available for the years 1976 and 1977. The figure is taken from Hajra et al. (2013). 


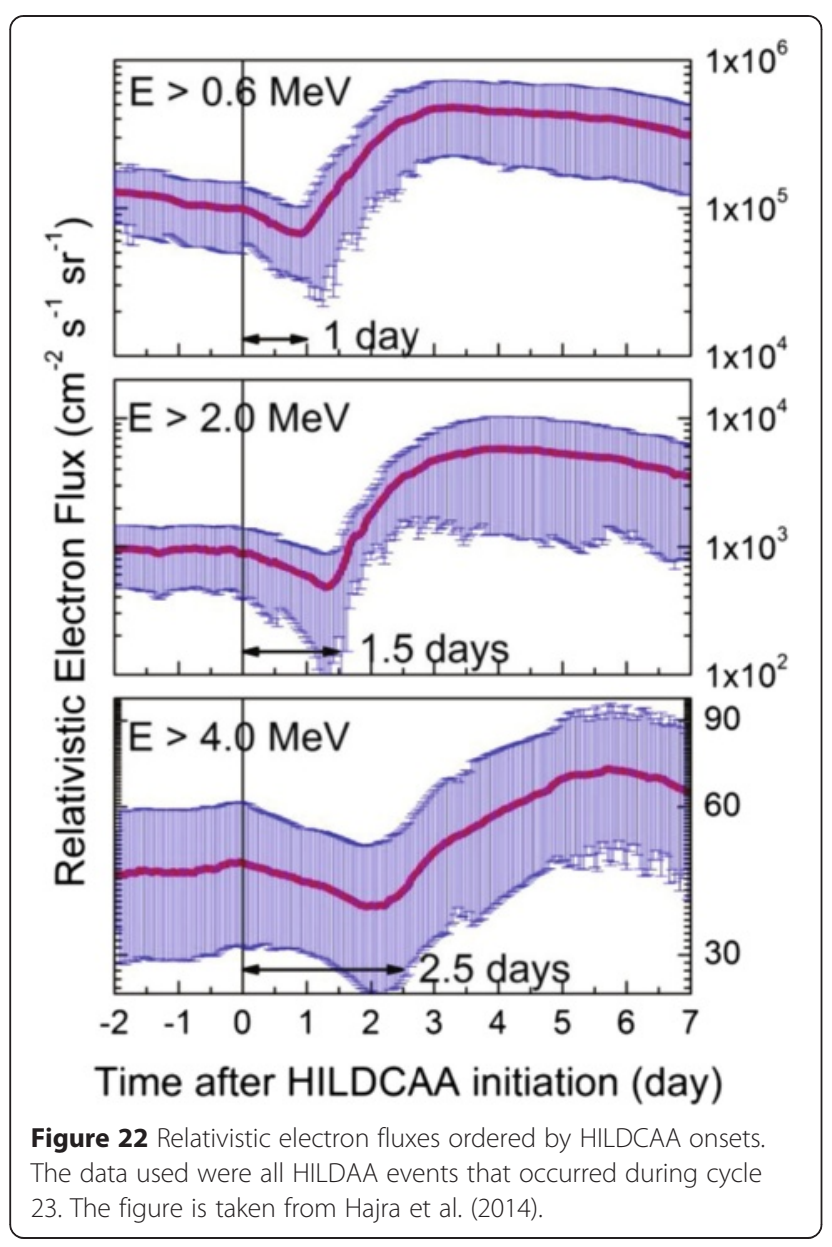

cycle dependence of these events for $3 \frac{1}{2}$ solar cycles is shown in Figure 21.

Figure 21 gives the solar cycle dependence of HILDCAAs. HILDCAAs are most often detected during the declining phase of the solar cycle, but they can be detected during the other phases as well.

Figure 22 gives the $\mathrm{E}>0.6-$, $>2.0-$, and $>4.0-\mathrm{MeV}$ electron fluxes detected at geosynchronous orbit. All of the 38 HILDCAA events that occurred during 1995 to 2008 (cycle 23) are included in this superposed epoch analysis. The start time is the onset of the HILDCAAs. For all of the events, the relativistic electron fluxes at the peak of the HILDCAA/HSS events were higher than their pre-event values Hajra et al. 2015. Thus, it is clear that acceleration had taken place and not simple removal and replacement of the electrons. It was also found that $100 \%$ of the events had simultaneous electromagnetic chorus waves present (when data were available) for the $5<\mathrm{L}<10$ and $00<\mathrm{MLT}<12$ region where chorus is expected to occur (Tsurutani and Smith 1977; Meredith et al. 2001, 2003). The figure shows that the $\mathrm{E}>$ $0.6-$, $>2.0-$, and $>4.0-\mathrm{MeV}$ electrons occurred approximately 1.0, 1.5, and 2.5 days after HILDCAA onsets. The results are in good agreement with the theoretical predictions (Horne and Thorne 2003, Horne 2007). Approximately 10 - to $100-\mathrm{keV}$ substorm-injected electrons generate chorus by the loss cone/temperature anisotropy instability. These same waves cyclotron resonate with approximately $100-\mathrm{keV}$ electrons to produce relativistic electrons on approximate day time scales. In this scenario, the $\mathrm{E}=0.6 \mathrm{-MeV}$ electrons are accelerated first, then the $\mathrm{E}=2.0-\mathrm{MeV}$ population from the $0.6-\mathrm{MeV}$ electrons and so forth. This 'boot strap' acceleration scenario was the interpretation of Hajra et al. (2014) for the various delay times of the relativistic electrons shown in the figure.

The overall scenario of the magnetospheric relativistic electron acceleration starts at the Sun (Tsurutani et al. 2006, 2010). Supergranular circulation is the source of the Alfvén waves (Hollweg 2006). These Alfvén waves are carried from the coronal holes at the Sun to the Earth by the HSSs. The southward components of the Alfvén waves lead to magnetic reconnection and the geomagnetic activity indicated in the HILDCAAs. The injection of approximately $10-$ to $100-\mathrm{keV}$ electrons by substorms/convection events within the HILDCAAs lead to chorus wave growth and the chorus accelerate electrons to relativistic energies. All features of this scenario have now been confirmed, except for the Alfvén wave source. Perhaps the Solar Probe Plus or Solar Orbiter will be able to finally confirm this last part of the scenario.

\section{CIR and HSS ionospheric and atmospheric effects}

Verkhoglyadova et al. (2011) studied ionospheric and atmospheric effects of HSSs in the solar minimum interval, 25 March to 26 April 2008. This was the study interval for the whole heliospheric interval (WHI) science team. As stated previously, this was near the solar minimum but about a year before the geomagnetic minimum.

Figure 23 top panel shows the TIMED SABER enhanced NO infrared radiation coming from the atmosphere during the WHI HILDCAA activity (third panel) during the magnetic storm recovery phases (bottom panel). These are the intervals of high AE activity. Seven wide latitude bins are indicated. Most of this irradiation is coming from high latitudes, presumably the auroral zone region.

Mlynczak et al. (2003) views NO irradiation as a 'natural thermostat.' Energy input into the upper atmosphere during high $\mathrm{AE}$ intervals is converted to heat and changes the distribution of $\mathrm{NO}$ in the thermosphere and its radiative properties. The radiation from $\mathrm{NO}$ cools the atmosphere. $\mathrm{NO}$ is believed to account for approximately $50 \%$ of the estimated energy input to the atmosphere from the magnetic storm. 


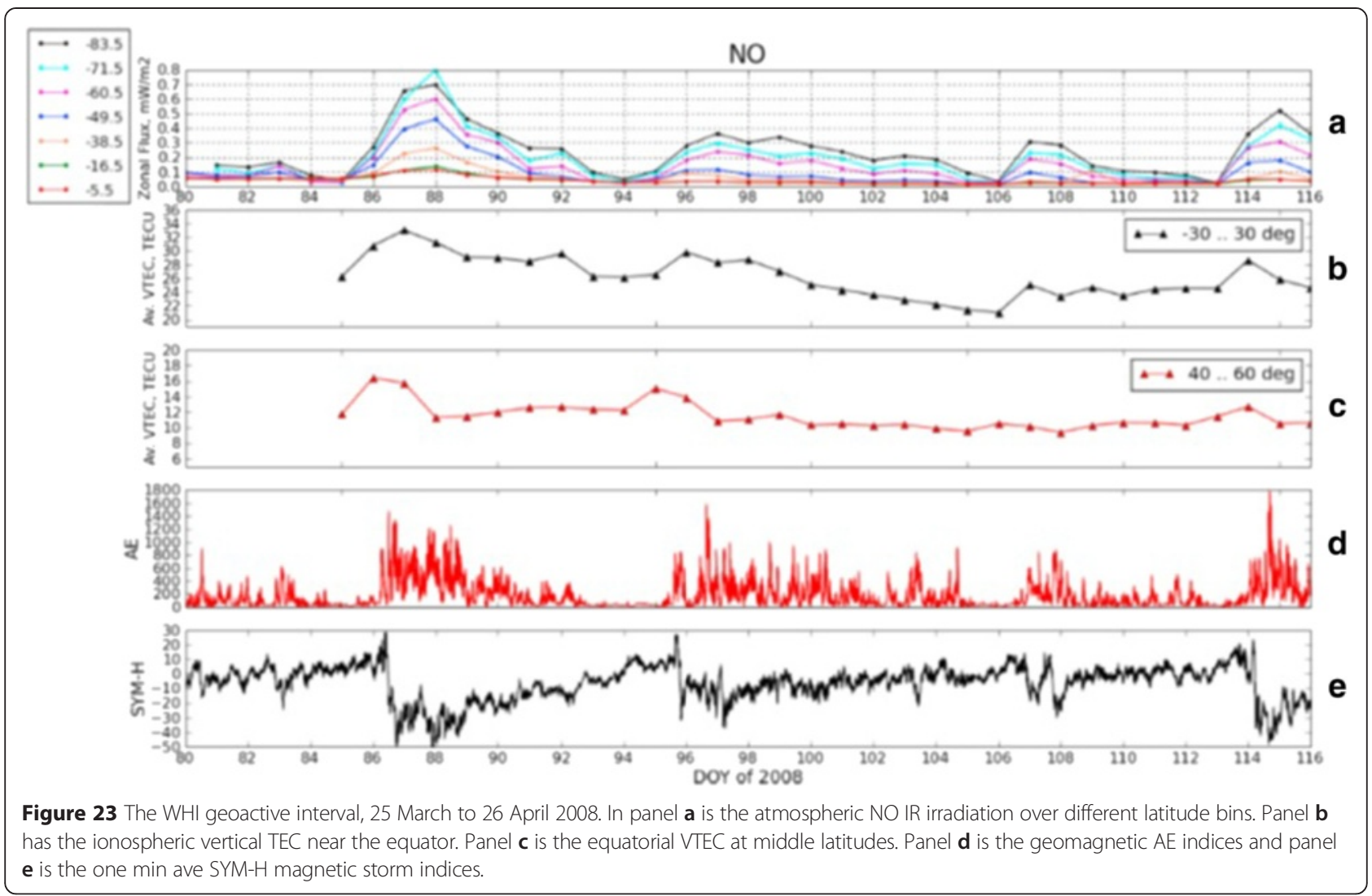

The ionospheric TEC effects during CIRs/HSSs are weak but are clearly present (Verkhoglyadova et al. 2011). There is enhanced TEC both at low latitudes (second panel: $-30^{\circ}<$ MLAT $<+30^{\circ}$ ) and at middle latitudes (third panel: $40^{\circ}$ to $60^{\circ} \mathrm{MLAT}$ ). The middle latitude disturbance is most likely due to particle precipitation in the auroral zone. However, the equatorial disturbances are less well understood. It may be associated with a disturbance dynamo effect or the dayside equatorial superfountain. More study is warranted.

\section{Microwave enhancement in coronal holes and solar wind speed}

Akiyama et al. (2013) also considered weak and strong CIRs but within solar cycle 23 (1996 to 2005). The weak and strong CIRs resulted in the Dst index $>-100 \mathrm{nT}$ and $<-100 \mathrm{nT}$, respectively. The CIRs were associated with low-latitude coronal holes identified in EUV images from SOHO/EIT. They measured the area of the coronal hole in EUV and in microwaves (from the Nobeyama Radioheliograph). Coronal holes appear bright in microwaves, and the area of microwave enhancement overlaps with that in EUV but not completely. The area of microwave enhancement is typically a third of the area observed in EUV CH on the average (Gopalswamy et al. 2000). There are at least two reasons for this difference:
(1) the microwave enhancement originates in the chromosphere, so the area is expected to be smaller due to magnetic field expansion, and (2) microwave enhancement is an indicator of higher magnetic field within the coronal hole, which is usually patchy within the coronal hole. A clear correlation between the $\mathrm{CH}$ size and the solar wind speed is well known (e.g., Nolte et al. 1976).

Figure 24 shows a linear correlation between the maximum solar wind speed and the $\mathrm{CH}$ area in EUV $(r=0.62)$ and microwaves $(r=0.79)$. The correlation is slightly better for the $\mathrm{CH}$ area in microwaves, suggesting that faster wind originates from higher magnetic field regions. Figure 24 also shows that the intense storms (Dst $<-100$ $\mathrm{nT}$ ) are associated with faster winds compared to the weaker storms (Dst $>-100 \mathrm{nT}$ ). This may simply be due to the fact that the Dst index depends on the product of solar wind speed and the southward component of the CIR magnetic field.

\section{The lone major CIR storm of cycle 24}

The only major CIR storm of cycle 24 (as of this writing) occurred on 1 June 2013. The source of the storm at the Sun was a low-latitude coronal hole (Figure 25). The storm was relatively intense (Dst $=-119 \mathrm{nT}$ ), with the strength similar to that of CME storms of cycle 24. The coronal hole was relatively large and straddled the 

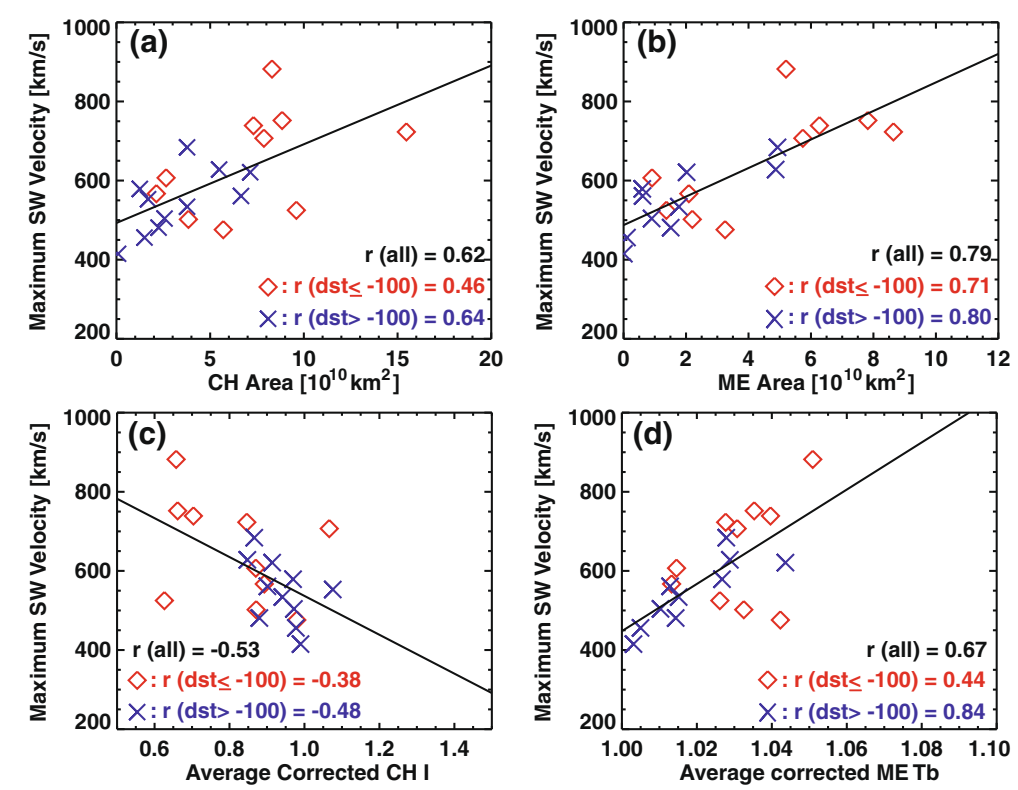

Figure 24 Scatter plots between solar wind (SW) speed and coronal hole parameters. (a) Coronal hole area in EUV, (b) coronal hole area that shows microwave enhancement (ME), (c) EUV intensity averaged over the coronal hole area, (d) and the microwave brightness temperature (Tb) averaged over the ME area (from Akiyama et al. 2013).

equator as can be seen from the SDO/AIA image taken in the beginning of 30 May 2013. An outline of the coronal hole superposed on the SDO/HMI magnetogram shows that it encloses a region of positive magnetic polarity. In situ observations by spacecraft at L1 show that the CIR had a large magnetic field (approximately 25 $\mathrm{nT}$ ) and the Bz was also large (approximately $-21 \mathrm{nT}$ ), which was responsible for the geomagnetic storm.

\section{Large SEP events and GLE events}

Solar energetic particles (SEPs) are part of the mass emission from the Sun, intimately connected to CMEs and flares. The current paradigm is that the material in the corona and IP medium is accelerated by CMEdriven shocks to produce the large SEP events (e.g., Reames 1999, 2013). Detailed investigation on the connection between CME-driven shocks and SEPs became possible only after the advent of SOHO coronagraphs that routinely imaged CMEs in the coronal region from where the energetic particles are released. In particular, the highest energy $(\mathrm{GeV})$ particles have been studied in relation to CMEs only for the past couple of solar cycles (Gopalswamy et al. 2012d; Nitta et al. 2012; Mewaldt et al. 2012; Miroshnichenko et al. 2013). Studies on the connection of SEPs to CMEs have been further enhanced by the extended coverage provided by STEREO in a number of ways, including observations all around the Sun. The heliospheric imagers and the inner coronagraph COR1 have extended the spatial domain over which CMEs are observed. In addition, EUV observations from STEREO/EUVI and SDO/AIA have improved our ability to study the early phase of CMEs (see e.g., Aschwanden et al. 2014). For example, the coronal height where shocks form can be readily determined from EUV and COR1 images, so that the time available for accelerating SEPs can be estimated accurately. Characterizing the size of large eruptions from behind the limb has been made possible by estimating the soft X-ray fluxes based on the known correlation between EUV flux and soft X-ray flux for frontside events (Nitta et al. 2013b).

Even though STEREO was launched by the end of 2006, no large SEP events occurred until the second half of 2010 in the CAWSES-II period. From then on, there were many SEP events that have been studied extensively. The STEREO particle detectors observed SEP events from off the Sun-Earth line and, together with detectors at Sun-Earth L1, provided information on the longitudinal distribution of SEP intensity (e.g., Dresing et al. 2012; Rouillard et al. 2012; Mewaldt et al. 2013). It also became possible to study the radial dependence of SEP intensities, thanks to the observations provided by MESSENGER spacecraft (Lario et al. 2013). Observations from multiple spacecrafts have also been used to test diffusive shock acceleration: Wang and Yan (2012) performed a dynamical Monte Carlo simulation of the CME-driven shock that occurred on 14 December 2006 using an anisotropic scattering law and found that the simulated results of the shock's fine structure, particle injection, and energy spectrum were in good agreement with the observations. 

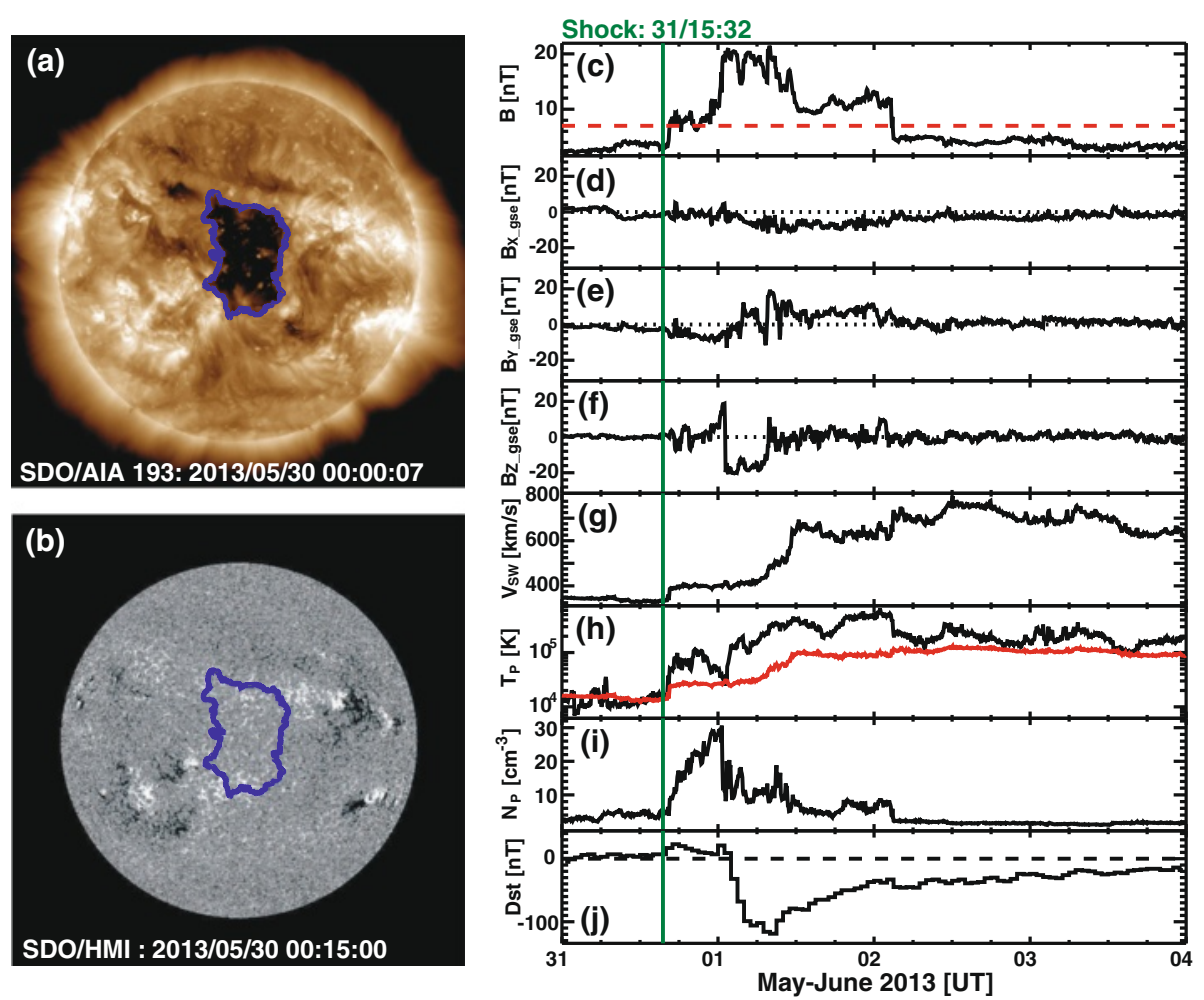

Figure 25 The first and only large CIR storm of cycle 24 as of this writing and its solar source region (coronal hole). The CIR was due to the high-speed stream from a large equatorial coronal hole (a) observed by SDO/AIA at $193 \AA$. At the photospheric level, the coronal hole had positive magnetic polarity (b) as observed by SDO/HMI. The outline of the coronal hole in (a) is overlaid on the magnetogram (b) to show the unipolar region. The CIR manifested as a region of enhanced magnetic field in in-situ observations (c). The three components of the IP magnetic field are shown in (d-f). The Bx component was negative indicating that the direction of the magnetic field was pointing away from the Sun consistent with the positive polarity in the photospheric magnetogram. The Bz component was negative for approximately $6 \mathrm{~h}$ and was responsible for the major storm. It should be noted that this type of magnetic structure in CIRs is atypical (see previous discussion). The solar wind speed increased from approximately $380 \mathrm{~km} / \mathrm{s}$ first as a shock jump and then at the interface and finally in the high-speed stream (g). The temperature remained higher than the expected solar wind temperature (shown in red) throughout the interface and the fast wind (h). The density in the interface was high due to the shock compression reaching values in the range 10 to $30 \mathrm{~cm}^{-3}$ (i). Finally, the Dst index shows the evolution of the storm, reaching a peak value of -119 nT on June 1 at 9 UT (j).

\section{Longitudinal dependence of SEP intensity}

Lario et al. $(2006,2013)$ were able to fit a Gaussian to the ensemble of multispacecraft SEP peak intensities as a function of the longitudinal distance between the solar source (inferred from flare observations) and the magnetic connection point for each spacecraft on the Sun. They found that such distributions are slightly offset to the west from the solar source. Long-lived particle injection from CME-driven shocks has been suggested as the reason for this shift (Lario et al. 2014). They also estimated that the shock height is within approximately 40 Rs when the particle injection from the shock maximizes. Given the observation that CME-driven shocks form at a heliocentric distance of 1.5 to 5 Rs (Tsurutani et al. 2003a; Gopalswamy et al. 2013b), these findings indicate that observations close to the Sun are most important for large SEP events. Furthermore, the range of heliocentric distances matches with the frequency range of IP type II bursts (Gopalswamy et al. 2012c).

\section{SEP intensity variability}

Attempts to understand the variability in SEP intensities have been focused on two aspects: (1) source factors involving CME properties, (2) environmental factors such as ambient magnetosonic speed, preceding CMEs, deflection by large-scale structures, seed particles, and turbulence. The CME speed and width are the primary source parameters. In cycle 23 , about approximately $75 \%$ of SEP-producing CMEs were found to be halos (Gopalswamy 2006b) for the whole cycle as well as for the first 62 months. On the other hand, 100\% of SEPproducing CMEs are halos in cycle 24 so far. This remarkable result can be understood in terms of the anomalous expansion of CMEs in cycle 24 due to the reduced heliospheric pressure. The average sky-plane speed of SEP-producing CMEs is approximately 1,500 $\mathrm{km} / \mathrm{s}$, slightly higher than that in cycle 23 over the corresponding phase of the solar cycle (Gopalswamy 2012). These results lead to the conclusion that the cycle-24 
CMEs need to have more kinetic energy to produce SEP events similar to those in cycle 23. In other words, the cycle-24 CMEs seem to be less efficient in accelerating particles.

\section{SEP events associated with weak and strong eruptions}

SEP events are generally associated with large soft X-ray flares, but this may not indicate a physical connection (Cliver et al. 2012). In fact, soft X-ray flare size is not a good indicator of SEP association. Gopalswamy et al. (2014b) investigated 59 major eruptions with flare size $\geq$ M5. The CME flux rope location was on the front side of the Sun for 55 of them. Only 20 (or 36\%) of the 55 eruptions were associated with large SEP events including those detected by STEREO-B (located behind the east limb). On the other hand, out of the 31 large SEP events detected by GOES during the first 62 months of cycle 24, ten were associated with $<$ M5.0 flares (half of them associated with C-class flares). SEP-associated CMEs in both these populations (size $\geq$ M5.0 and $<$ M5.0) were very fast: the average speeds were 2,300 and $1,720 \mathrm{~km} / \mathrm{s}$, respectively. This result is consistent with the idea that energetic particles in large SEP events are primarily accelerated by CME-driven shocks, as was pointed out by and Gopalswamy et al. (2012d) for GLE events. This point is further illustrated in Figure 26, which shows the source locations of the 55 major eruptions ( $\geq$ M5.0) of cycle 23 and the ones with 20 large SEP events. The eruptions are divided into two groups according to their speed $(\geq 1,500$ $\mathrm{km} / \mathrm{s}$ for 28 events and $<1,500 \mathrm{~km} / \mathrm{s}$ for 27 events). Out of the 28 fast CMEs, 17 (or 61\%) were associated with large SEP events, compared to only 3 (or 11\%) of the 27 slower CMEs. Figure 26 also shows the solar sources of SEP events associated with the ten weaker eruptions $(M<5.0)$ : only two (or 20\%) of the weak eruptions were associated with $<1,500 \mathrm{~km} / \mathrm{s}$ CMEs while eight (or $80 \%$ ) were associated with $\geq 1,500 \mathrm{~km} / \mathrm{s}$ CMEs. The faster CMEs without SEP events mostly occurred when there was high background level of SEPs due to previous events. In one case, the CME was jet like (the width was $<<60^{\circ}$ ).

\section{Solar cycle effects}

Unlike major geomagnetic storms, the number of large SEP events did not decrease significantly in cycle 24 . Figure 27 shows the number of large SEP events binned into Carrington rotations from September 1995 (end of cycle 22) to April 2014 (middle of cycle 24). The gap between the last event of cycle 22 and the first event of cycle 23 was approximately 2 years. The corresponding gap was nearly doubled during the cycle 23/24 minimum. However, once the activity started, the number of large SEP events occurred roughly at the same rate as in the first 5 years of cycle 23. As of June 2014, there were 34 large SEP events in cycle 24 compared to 47 over the corresponding phase in cycle 23 . The reduction is only by $26 \%$, similar to the reduction in the number of fast and wide western CMEs (W20 to W90) in cycle 24 (Gopalswamy et al. 2014a).

The peak intensity and fluence of the $>10-\mathrm{MeV}$ SEP events in cycle 24 were not too different from the corresponding values in cycle 23 (Figure 28). This is in stark contrast to the major reduction in the intensity and number of major geomagnetic storms in cycle 24. The SEP behavior can also be explained in terms of the altered state of the heliosphere: as Figure 13 shows, the Alfvén speed of the corona decreased during the rise phase of cycle 24 compared to the corresponding phase in cycle 23. Lower upstream magnetosonic speeds result in higher Mach number shocks for a given CME speed, increasing the likelihood of the occurrence of large SEP

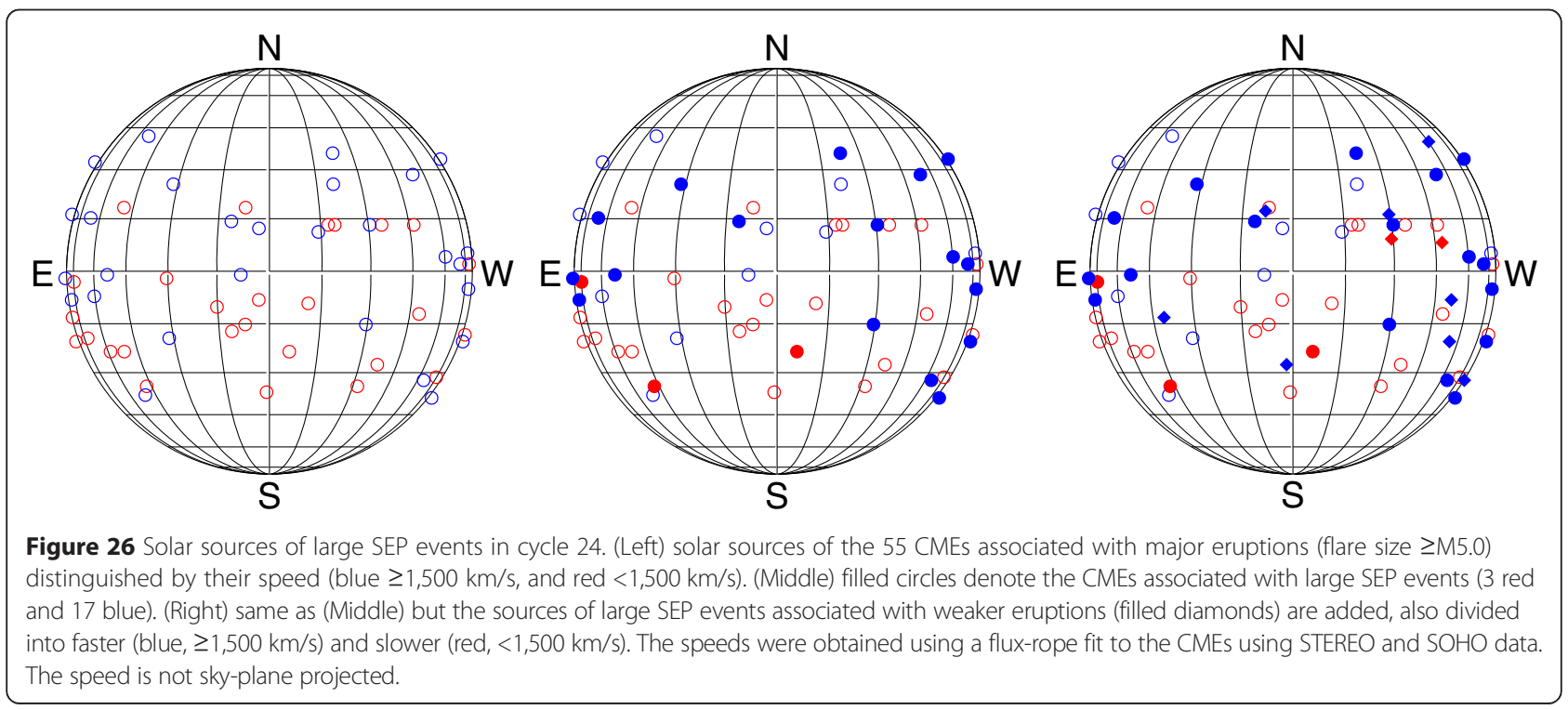




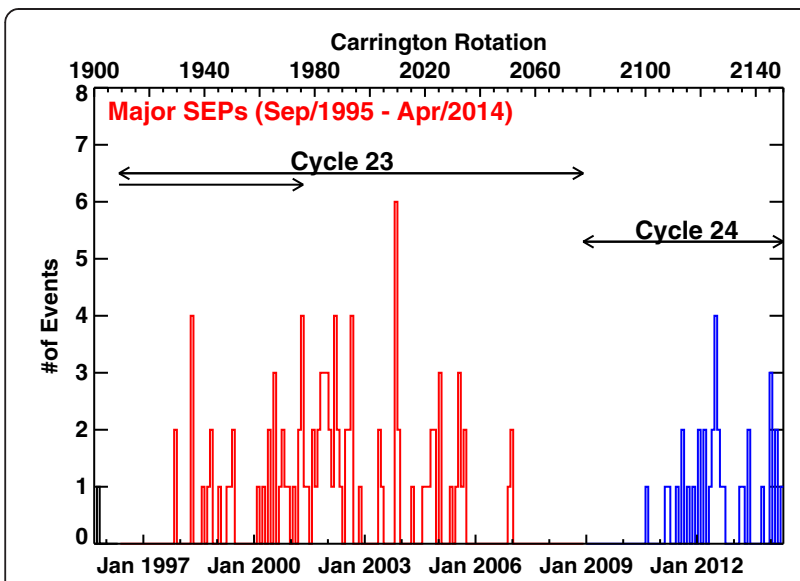

Figure 27 Number of large SEP events as a function of time from September 1995 to the end of April 2014. The black, red, and blue plots denote cycles 22, 23, and 24, respectively. The right-point arrow within the cycle-23 interval corresponds to the first 66 months of cycle 23 (May 1996 to November 2001) used for comparing with the current length of cycle 24 (December 2008 to April 2014). The first large SEP event occurred only in August 2010.

events. The two solar cycles, however, differ drastically in the case of higher energy SEP events. The number of large SEP events with $>500-\mathrm{MeV}$ particles declined by $58 \%$, while the number of GLE events declined by $71 \%$. These cannot be explained by the $22 \%$ drop in the number of fast and wide CMEs in cycle 24. This issue is addressed in the next section.

It should be noted that the largest SEP events are associated with quasi-parallel shocks (Kennel et al. 1984a, 1984b). This is because of the presence of upstream turbulence ahead of the shocks (Tsurutani et al. 1983), leading to enhanced Fermi-type acceleration across the shocks.

\section{The paucity of GLE events in cycle 24}

Ground-level enhancement (GLE) in SEPs represent the highest energy particles accelerated by the Sun, making the particles penetrate Earth's atmosphere to the troposphere where they produce air showers like those produced by the galactic cosmic rays (GCRs) and the air showers are detected by ionization chambers, muon detectors, and neutron monitors on the ground. These hard-spectrum events affect navigation systems, spacecraft electronics and operations, space power systems, manned space missions, and commercial aircraft operations (Shea and Smart 2012; Mewaldt et al. 2012; Kudela 2013; Ruffolo et al. 2013). In particular, they can be a significant radiation exposure to humans in space and in airplanes on polar routes. GLE events also have important implications for VLF wave propagation: Zigman et al. (2014) found that at energies up to approximately $2 \mathrm{GeV}$, the ionization rate for solar protons may exceed the GCR ionization by 1.5 orders of magnitude.

Typically about a dozen GLEs occur in each solar cycle, which is about $15 \%$ of the number of large SEP events during cycles 19 to 23 (Shea and Smart 2008). However, there were only two GLEs during cycle 24 so far, even though there were 34 large SEP events, amounting to $<6 \%$ (Gopalswamy et al. 2013a; Thakur et al. 2014; Papaioannou et al. 2014). The paucity of GLE events in cycle 24 cannot be explained by the $22 \%$ reduction in the number of fast and wide CMEs originating in the traditional GLE longitudes (W20 to W90). A combination of several factors is needed to explain the drastic reduction in the number of GLE events: (1) The reduction in the ambient magnetic field in the corona reduces the efficiency of shock acceleration especially for $\mathrm{GeV}$ particles, even though it is not a problem in accelerating approximately $10-\mathrm{MeV}$ particles. (2) Many CMEs have nonradial motion either due to the coronal environment or inherent asymmetry in the source region. The nonradial motion makes the shock nose not well connected to Earth (Gopalswamy et al. 2013a; Gopalswamy and Mäkelä 2014). This means that even if $\mathrm{GeV}$ particles were accelerated at the shock nose, they may not reach

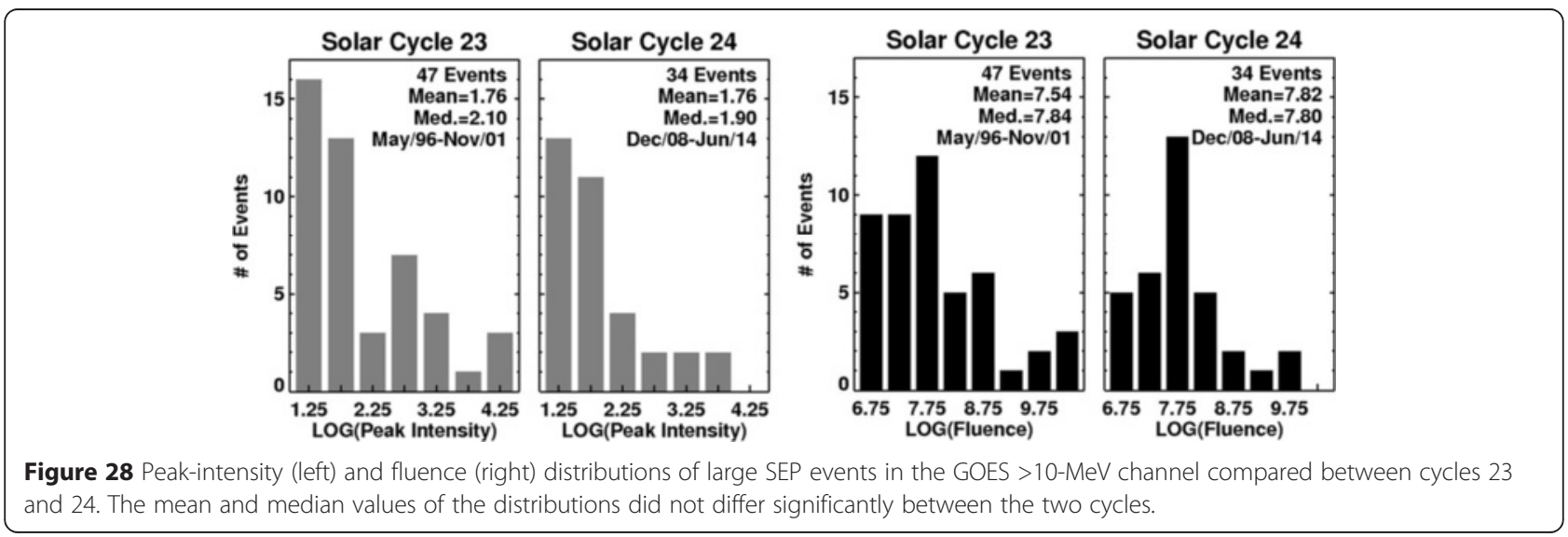




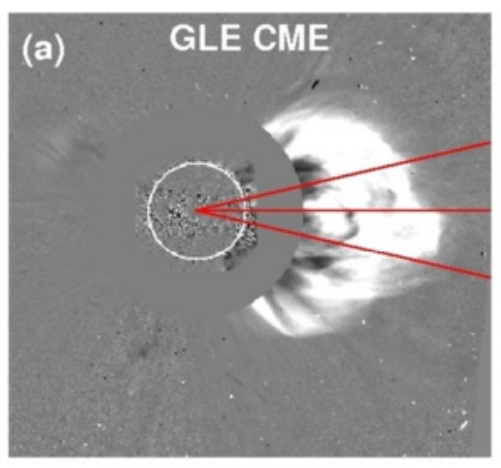

SOHO: 2014/01/06 08:12

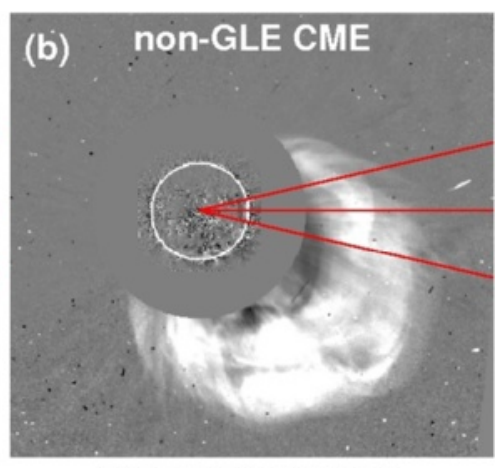

SOHO: 2014/01/07 18:36

Figure 29 SOHO/LASCO snapshots of two CMES, one associated with a GLE event (left) (a) and the other with only a large SEP event (b). The January 6 CME occurred slightly behind the limb. The January 7 CME originated from close to the disk center. The three red lines mark the ecliptic (central line) and the edges of the ecliptic distance range $\left( \pm 13^{\circ}\right.$ ) over which GLE CMEs occurred in cycle 23. The LASCO images are superposed on SDO/AIA difference images at $193 \AA$, which show the disturbances behind the west limb for the January 6 CME and close to the disk center for the January 7 CME. (Adapted from Gopalswamy et al. 2014b).

the observer. (3) The CME may be ejected into a locally tenuous corona making the shock weaker.

Figure 29 compares the 6 January 2014 GLE CME (Thakur et al. 2014) with a non-GLE CME on January 7 that was even faster than the GLE CME. The nose of the January 6 CME was in the ecliptic; it was well below the ecliptic for the January $7 \mathrm{CME}$. In other words, the nose of the January 6 shock was magnetically connected to Earth, while just the flank of the shock was connected during the January 7 event. The 6 January 2014 CME resulted in a GLE even though the speed was smaller than the January $7 \mathrm{CME}(1,700 \mathrm{~km} / \mathrm{s}$ vs. $3,100 \mathrm{~km} / \mathrm{s})$. The January 6 CME originated from behind the limb (S02W102) while the January $7 \mathrm{CME}$ originated from close to the disk center (S15W29). The nonradial motion in CMEs can be attributed to deflection by nearby coronal holes (Gopalswamy 2009d; 2010d, Kay et al. 2013) or to the inherent asymmetry in the distribution of magnetic field strength in the source region (see e.g., Sterling et al. 2011). In the source region of the January $7 \mathrm{CME}$, the flare ribbons were located to the south of intense fields that did not participate in the eruption but might have deflected the CME to the south. There was also a coronal hole to the northeast of the eruption region, which might have also contributed to the deflection.

The fact that both CMEs in Figure 29 were large SEP events raises an important question on the location of particle acceleration on the shock surface. The stringent requirement of the ecliptic distance for GLE CMEs suggests that GLEs may be accelerated at the shock nose, where the shock is the strongest. On the other hand, magnetic connection to any part of the shock seems to suffice for large SEP events. This means that lower energy SEPs are accelerated over most of the shock surface. This is also consistent with the study by Dalla and
Agueda (2010) who found that the probability of detecting SEP events remained constant up to a latitude of $28^{\circ}$. Gopalswamy and Mäkelä (2014) examined the latitudinal connectivity issue in historical GLE source regions that were at latitudes $>30^{\circ}$. They were able to show that in all the higher latitude cases, there was a polar coronal hole, a streamer, or a pseudo-streamer poleward of the GLE source region suggesting deflection toward equator and hence enhancing the possibility of shock nose connection to Earth.

The requirement that the CME nose be in the ecliptic for GLE events may also have implications for the shock geometry. It is reasonably certain that GLEs are released when the CME is at a height of approximately 3 Rs. Since the shock nose has the largest heliocentric distance compared to other parts, it is likely that the nose is above the source surface and the upstream field magnetic field is open. This suggests that GLE-producing regions of the shock may have quasi-parallel geometry. Recent investigations confirm the suggestion that CME-driven shocks are likely to be supercritical and quasi-parallel near the nose, while subcritical and quasi-perpendicular at the flanks (Bemporad et al. 2014; Bemporad and Mancuso 2011, 2013). It is well known that the first critical Mach number is the smallest (approximately 1.5) for quasi-parallel shocks and the largest for quasi-perpendicular shocks (approximately 2.7) (see e.g., Mann et al. 2003). Supercritical shocks are known to accelerate particles more efficiently (Burgess and Scholer 2013). Thus for a given shock speed (approximately 2,000 km/s for GLE events) and the typical Alfvén speed of approximately $600 \mathrm{~km} / \mathrm{s}$ at 3 Rs (Gopalswamy et al. 2001b), the quasi-parallel shocks are likely to be definitely supercritical, while the quasi-perpendicular shocks may or may not be supercritical. These conclusions on the shock geometry and 
connectivity need to be further explored and modeled for a full understanding of particle acceleration by CMEdriven shocks.

\section{Extreme space weather}

Given that CMEs are responsible for the largest geomagnetic storms and SEP events, it is natural to think that some properties of CMEs or their source regions may result in extreme events. If we define extreme events as those, which lie on the tail of a distribution, we can readily identify the CME's speed as one critical parameter. From the cumulative distribution of CME speeds, one infers that there are not many CMEs with speeds exceeding approximately $3,500 \mathrm{~km} / \mathrm{s}$ (e.g., Gopalswamy et al. 2010b). When a CME has a speed $>3,500 \mathrm{~km} / \mathrm{s}$, it may be thought of as an extreme event. The consequences of such an energetic CME are likely to be extreme also. An extremely fast CME will drive a shock, which will accelerate particles to very high energies. If all conditions for the acceleration of particles are conducive (low ambient magnetosonic speeds, good connection of the shock nose to the observer, a quasi-parallel shock, and a preconditioned ambient medium), one might expect an extreme SEP event. Kovaltsov and Usoskin (2014) determined the cumulative occurrence probability distribution of SEP events based on directly measured SEP fluences for the past 60 years, estimates based on the terrestrial cosmogenic radionuclides ${ }^{10} \mathrm{Be}$ and ${ }^{14} \mathrm{C}$ for Holocene time scale, and cosmogenic radionuclides measured in lunar rocks on a time scale of up to 1 Myr. They concluded that SEP events with a $>30-\mathrm{MeV}$ proton fluence greater than $10^{11}$ (protons $\mathrm{cm}^{-2}$ year $^{-1}$ ) are not expected on a Myr time scale.

The SEP event depends just on the outer structure of a fast CME, viz., the shock. It should be noted that the most intense interplanetary shock detected at 1 AU distance from the sun was detected on March 8 in the 7 to 17 March 2012 CAWSES-II study interval and had a magnetosonic Mach number of approximately 9.4 (Tsurutani et al. 2014). However, the maximum shock Mach number is possible, assuming an ICME speed of $2,700 \mathrm{~km} / \mathrm{s}$ is 45 (Tsurutani and Lakhina 2014). One speculation to explain the difference is that it is possible that the acceleration of energetic particles at the shock leads to 'shock damping,' reducing the shock intensity. Theoretical studies are needed to confirm/deny this hypothesis.

On the other hand, the generation of a large geomagnetic storm depends on the internal structure of the CME, especially the magnetic cloud (MC, Burlaga et al. 1982) and filament (Kozyra et al. 2014) internal to it. What is important is that the magnetic field intensities are high and southward. It has been shown empirically that fast CMEs have particularly intense magnetic fields (Gonzalez et al. 1998). Sheath magnetic fields (Tsurutani et al. 1988) are swept up slow solar wind fields and not part of the CME proper. Sheath fields can have intensities of only approximately four times the upstream ambient field strength (Kennel et al. 1985; Tsurutani and Lakhina 2014) unless multiple shocks are compressing the sheath, so this region is, in general, unimportant for the cause of extreme storms. If the magnetic cloud has its axis pointing to the south and the magnetic field is high, one could expect strong magnetic interconnection between the interplanetary magnetic field and the Earth's magnetopause magnetic field (Dungey 1961) and therefore an intense geomagnetic storm. In addition, if the shock remained strong, it would produce an extreme impulse on the magnetosphere that can expose geosynchronous orbit to the solar wind (Tsurutani and Lakhina 2014) and a sudden (positive) impulse $\left(\mathrm{SI}^{+}\right)$on the ground (Tsurutani et al. 2008). An intense shock would also produce an extreme energetic storm particle (ESP) event at Earth (Tsurutani et al. 2009b). Thus for solar terrestrial relationships, an extreme event in its origin will also have extreme consequences at Earth and nearEarth space environment.

Tsurutani and Lakhina (2014) considered an extreme scenario for geomagnetic response. Since the CME speed and its magnetic content can ultimately be traced to the solar source (Gopalswamy 2010), an extreme CME would be born in an active region with enormous free energy. Going by the highest field strength ever observed in a sunspot (approximately 6,100 G, Livingston et al. 2006) and the largest active region area $(5,000 \mathrm{msh}$, Newton 1955), one can estimate a free energy of approximately $10^{36} \mathrm{erg}$. This is two to three orders of magnitude larger than the amount of free energy estimated for NOAA AR 10486, which produced several of the Halloween CMEs (Gopalswamy et al. 2005c). If the entire approximately $10^{36}$ erg goes into the kinetic energy of a CME of mass approximately $10^{18} \mathrm{~g}$, then the CME speed would be approximately $14,000 \mathrm{~km} / \mathrm{s}$, about four times larger than the CME speed ever observed.

Recently, Maehara et al. (2012) investigated 365 stellar flares with energies in the range $10^{33}$ to $10^{35}$ erg using the Kepler mission data. Fourteen of those flares were from Sun-like stars (G-type main sequence stars with rotational periods $>10$ days and surface temperature in the range 5,600 to $6000 \mathrm{~K}$ ). They estimated that superflares with energy $10^{35}$ erg occur once in 5,000 years. Note that this energy is an order or magnitude less than the maximum free energy estimated by Gopalswamy (2010). It must be pointed out that CMEs are not observed directly in stellar eruptions, but if the eruption mechanism is similar to that on the Sun, one would expect even higher CME kinetic energies than the $10^{35}$ erg flare energy found by Maehara et al. (2012). In fact, the CME kinetic energy gains the largest share among various 
ways in which the free energy in an active region is divided (Emslie et al. 2012). However, one should take note that the amount of energy that goes into the flare particles is deducted from the CME energy (Tsurutani and Lakhina 2014).

Shibata et al. (2013) also investigated theoretically the possibility of superflares on the Sun using the current dynamo model and concluded that it is indeed possible to generate magnetic flux necessary for producing superflares of energy in the range $10^{34}$ to $10^{35} \mathrm{erg}$ within the next 40 years. The Solar Evolution and Extrema (SEE) project of the VarSITI (Variability of the Sun and Its Terrestrial Impact) program of SCOSTEP is expected to make further progress on this issue over the next few years.

The Carrington flare of 1859 remains one of the important benchmark events for extreme solar events. The flare itself was detected with naked eyes, indicating that it is certainly an unexpected event. Cliver and Dietrich (2013) estimated that the soft X-ray flare size of X45, compared to X35 for the cycle-23 flare on 4 November 2003. The Carrington eruption did produce a historical geomagnetic storm, the highest intensity on record: Dst $=-1,760$ $\mathrm{nT}$ (Tsurutani et al. 2003b). Kataoka (2013) estimated that the probability of a Carrington-type geomagnetic storm occurring within the next decade is approximately $4 \%$ to $6 \%$. Yermolaev et al. (2013) performed a statistical analysis of the OMNI data for the period 1976 to 2000 and concluded that a Carrington-type event could occur once every 500 years (see also Alves et al. 2011). From the flare onset to the geomagnetic storm onset, Carrington (1859) gave a time of approximately $17.5 \mathrm{~h}$ from the flare to the storm, which indicates a CME speed of approximately 2,360 km/s (Gopalswamy et al. 2005c). The all-time record for the shortest CME transit time from the Sun to $1 \mathrm{AU}$ was the 12 August 1972 event (Vaisberg and Zastenker 1976) which was approximately $14.6 \mathrm{~h}$. With such a fast transit time and large magnetic cloud magnetic field, why did not a large magnetic storm occur? It was discovered that the magnetic field orientation within the magnetic cloud was northward, so the Earth had geomagnetic quiet rather than a large storm (Tsurutani et al. 1992a). It has been shown that for northward IMFs impinging on the Earth's magnetosphere, one has an extreme geomagnetic quiet rather than an intense storm (Tsurutani et al. 1992b; Du et al. 2011). These CME transit times are relatively close to the maximum time of $12 \mathrm{~h}$, assuming an initial CME speed of 3,000 km/s (Tsurutani and Lakhina 2014). Two of the Halloween storms in cycle 23 had such short transit times: 18.9 and $19.7 \mathrm{~h}$ (Tsurutani et al. 2005; Mannucci et al. 2005) for the 28 October 2003 and 29 CMEs, respectively. In the extremely weak cycle 24 , there was another extreme event on 23 July 2012 observed by multiple spacecrafts. This well-observed event merits some additional discussion.

\section{July 2012 event}

On 23 July 2012, the GOES satellite reported a large SEP event with a peak $>10-\mathrm{MeV}$ flux of approximately $12 \mathrm{pfu}\left(1 \mathrm{pfu}=1\right.$ particle $\left.\mathrm{sr}^{-1} \mathrm{~cm}^{-2} \mathrm{~s}^{-1}\right)$, a very ordinary event. However, the solar source of the associated CME was approximately $45^{\circ}$ behind the west limb and the CME was heading roughly toward STEREO-A (located at W121), which observed the CME as a full halo. STEREO-A also detected a 5,000-pfu SEP event with an ESP event approximately ten times larger, similar to the 23 March 1992 and 20 October 1989 events (43,000 and 40,000 pfu, respectively). The CME arrived at STEREOA in approximately $19 \mathrm{~h}$, making it one of the historical events potentially greater than the Halloween 2003 events (Russell et al. 2013b; Baker et al. 2013; Mewaldt et al. 2013; Gopalswamy et al. 2014b). The STEREO-A SEP event started approximately $4 \mathrm{~h}$ earlier than the GOES event and approximately $8 \mathrm{~h}$ earlier than the STEREO-B event because STEREO-A was better connected to the source than the other two spacecraft. There are a number of similarities between the 23 July 2012 event and the 20 October 1989 event studied by Lario and Decker (2002).

If the CME were heading toward Earth instead of STEREO-A, it would have caused another geomagnetic storm of historical proportions (Liu et al. 2014b). The total magnetic field strength was approximately $109 \mathrm{nT}$ in the shock sheath and approximately $60 \mathrm{nT}$ in the ICME. The southward component had a peak value of $-52 \mathrm{nT}$. Liu et al. (2014) estimated that the Dst index to be in the range $-1,150 \mathrm{nT}$ to $-600 \mathrm{nT}$. The simple empirical relation reported by Gopalswamy (2010) also gives a high Dst index: Dst $=-0.01 \mathrm{VBz}-32 \mathrm{nT}$. Plugging in $\mathrm{V}=1,500 \mathrm{~km} / \mathrm{s}$ and $\mathrm{Bz}=$ $52 \mathrm{nT}$, the Dst index becomes $-812 \mathrm{nT}$. This is half of the strength reported for the Carrington storm $(-1,760 \mathrm{nT}$, Tsurutani et al. 2003b) and a quarter of the maximum possible of Dst $=-3,500 \mathrm{nT}$ (Tsurutani and Lakhina 2014). That is if the magnetosphere does not saturate at Dst $=-2,500 \mathrm{nT}$ as Vasyliunas (2011) has argued. If we compare with the estimate of Siscoe et al. (2006), who used hourly averages to estimate the Dst index as -850 $\mathrm{nT}$, we see that the July 2012 storm has the same strength as the Carrington event (see also Cliver and Dietrich 2013 who estimate Dst approximately $-900 \mathrm{nT}$ ). The important point is that such extreme storms can occur even during the subdued heliospheric conditions prevalent in cycle 24 . It is possible that the July 2012 storm already represents a reduction in $\mathrm{Bz}$ because of the anomalous CME expansion; otherwise the storm could have been even bigger than the current estimates. If the ICME were south pointing with the 109-nT field during the July 2012 event (Liu et al. 2014) and the speed were similar to the shock speed $(2,250 \mathrm{~km} / \mathrm{s}$ ), the empirical formula (Dst $=0.01 \mathrm{VBz}-32$ $\mathrm{nT}$ ) would predict a storm of approximately $-2,500 \mathrm{nT}$. 


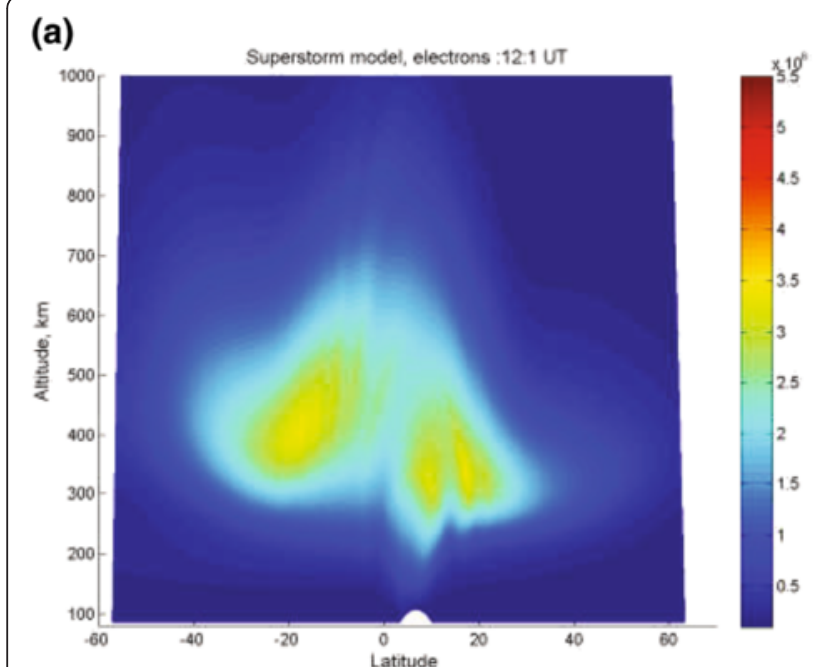

(c)

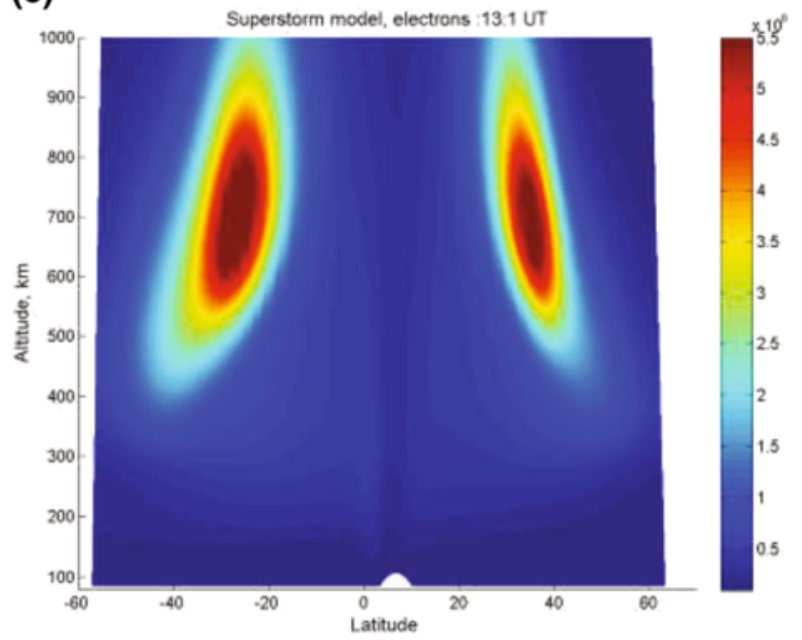

(b)

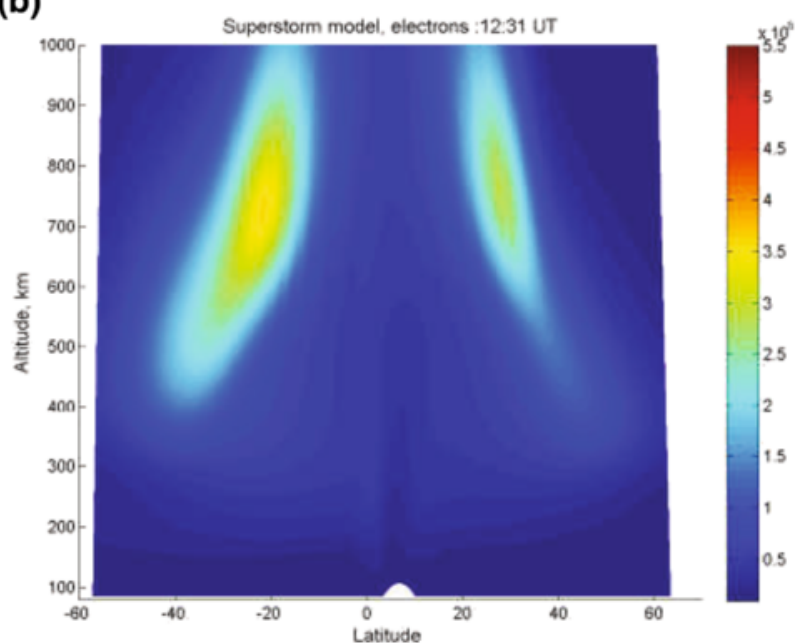

(d)

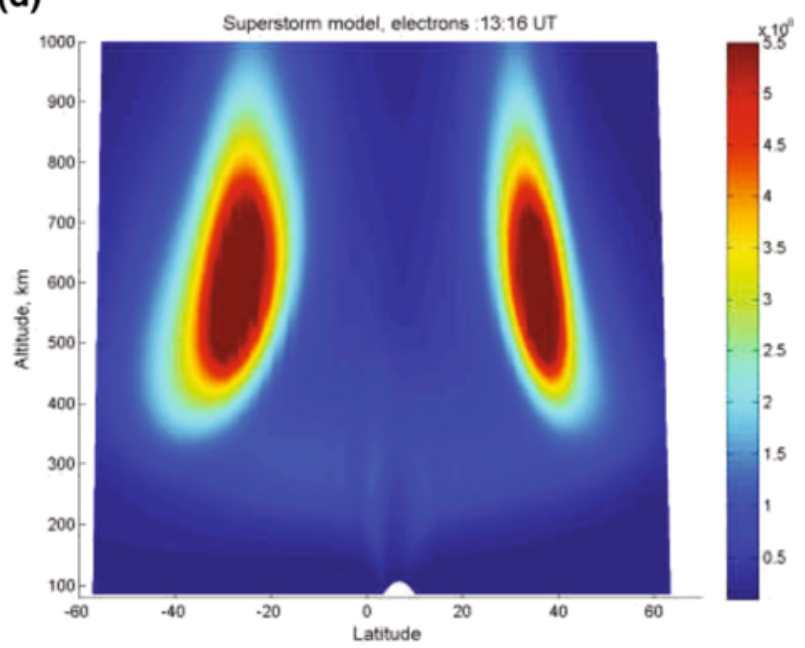

Figure 30 The oxygen ion profiles. (a) Prior to, (b) 30 min after, (c) 1 h after, and (d) 15 min after termination of the penetration of the interplanetary electric field. The figure is taken from Tsurutani et al. (2012).

\section{Carrington ionospheric event}

Scientists now understand the dayside superfountain effect well and can model such events. Figure 30 shows a simulation of the Carrington magnetic storm effects in the dayside ionosphere. The Huba et al. (2002) SAMI-2 code was used which describes the dynamics and chemical evolution of seven ion species and seven corresponding neutral species. The code solves collisional two-fluid equations for electrons and ions along dipole magnetic fields taking into account photoionization of neutrals, recombination of ions and electrons, and chemical reactions. The code was modified to allow an external electric field input (Verkhoglyadova et al. 2008) and was recently further modified to insert 3-h ap indices instead of daily values. A $20-\mathrm{mV} / \mathrm{m}$ electric field for a duration of $1 \mathrm{~h}$ was assumed (Tsurutani et al. 2003b, 2011b).
In panel a, prior to the event, the two EIAs are clearly noted one spanning approximately $-5^{\circ}$ to $-30^{\circ}$ and a second from approximately $+5^{\circ}$ to $20^{\circ}$ LAT (we use a $3.25 \times$ $10^{6}$ ions $/ \mathrm{cm}^{-3}$ to define the enhancement areas). Panel c shows that the oxygen peaks are located at approximately 530 to $920 \mathrm{~km}$ and approximately 500 to $900 \mathrm{~km}$ for the southern and northern regions, respectively. The magnetic latitude ranges are approximately $-20^{\circ}$ to $-35^{\circ}$ LAT and $+30^{\circ}$ to $+45^{\circ}$ LAT. The peak values reach approximately $6 \times 10^{6} \mathrm{O}^{+} \mathrm{cm}^{-3}$ at altitudes centered at approximately $700 \mathrm{~km}$. It is noted that the densities of the uplifted EIA peaks at $850 \mathrm{~km}$ and $1,000 \mathrm{~km}$ were approximately $4 \times 10^{6}$ and approximately $3.5 \times 10^{6} \mathrm{~cm}^{-3}$, respectively. These latter ion densities are substantially greater than quiet-time neutral density values, being approximately 40 times at $850 \mathrm{~km}$ and approximately 300 
times at 1,000 km. Thus, low altitude satellite drag will be substantially increased in such an extreme storm.

\section{Conclusions}

The weak activity as the Sun climbed toward the maximum phase of solar cycle 24 has provided an enormous opportunity to study the Sun and the heliosphere under quieter conditions. The space weather is less severe compared to cycle 23 in terms of intense geomagnetic storms and powerful SEP events. On the other hand, the cosmic-ray intensity remains exceptionally high after reaching the highest levels in the space age during the extended solar minimum. The geomagnetic ap index was the lowest on record. The latter was not only due to the changes of the Sun but also the location of the Earth relative to solar active regions (coronal holes). The vast array of ground- and space-based instruments has helped define this altered state of the Sun and heliosphere. The CAWSES-II program enabled many researchers to take the system approach and be mindful of the implications of their research for solar terrestrial relationship. From the eruptive events during the weak solar cycle to the dynamo problem and the grand minima of solar activity, the Sun has triggered a plethora of research activities, including the MiniMax24 activity that recognizes the importance of studying weak activity. The VarSITI program will address the declining phase of the weak cycle 24, when there will be phase-specific phenomena such as frequent CIRs and high-speed streams from the Sun.

The TG3 activities of the CAWSES-II program will naturally connect to the International Studies of Earthaffecting Transients (ISEST) project of VarSITI. Campaigns are being conducted in observing transient solar events under the MiniMax24 component of the ISEST project. The working groups under ISEST will deal with the theory, modeling, and detailed analysis of the campaign events. There will also be a close connection to the Solar Evolution and Extreme (SEE) project in examining flares on sun-like stars and extreme events such as the Carrington event and the 23 July 2012 event. These connections provide continuity to the SCOSTEP science activities and make progress without serious interruptions.

\footnotetext{
Abbreviations

AIA: atmospheric imaging assembly; AU: astronomical unit; CAWSES-Il: climate and weather of the Sun-Earth system II phase; CAT: CME analysis tool; CDAW: coordinated data analysis workshop; $\mathrm{CH}$ : coronal hole; CIR: corotating interaction region; CME: coronal mass ejection; EUV: extreme-ultraviolet; FD: Forbush decrease; FOV: field of view; GCR: galactic cosmic rays; GLE: ground level enhancement; GOES: Geostationary Operational Environmental Satellite: HI: heliospheric imager; HILDCAA: high-intensity long-duration continuous geomagnetic activity; HSS: high-speed stream; ICME: interplanetary coronal mass ejection; IP: interplanetary; LASCO: large angle and spectrometric coronagraph; MC: magnetic cloud; MHD: magnetohydrodynamics; SCOSTEP: Scientific Committee on Solar Terrestrial Physics; SDO: Solar Dynamics Observatory; SEPs: solar energetic particles; SGD: solar geophysical data; SOHO: Solar and Heliospheric Observatory; SSN: sunspot number;
}

STEREO: Solar Terrestrial Relations Observatory; TEC: total electron content; TG3: Task Group 3 (of CAWSES-II); TSI: total solar irradiance.

\section{Competing interests}

The authors' declared that they have no competing interest. All institutional and national guidelines for the care and use of laboratory animals were followed.

\section{Authors' contributions}

NG created the overall structure of the review article and was approved by the co-authors. He was responsible for drafting the full manuscript. BT contributed to several sections, especially on the geospace aspects. YY wrote some sections in the manuscript and coordinated the effort to check all the references. All authors read and approved the final manuscript.

\section{Authors' information}

NG is a NASA scientist at the Goddard Space Flight Center and the current President of SCOSTEP. As the President, he was responsible for the successful completion of the CAWSES-II program to which the present review belongs. NG is interested in the study of the origin, propagation, and Earth impact of solar disturbances. BT is a JPL scientist heavily involved in space plasma physics and the geospace impact of solar disturbances. YY is a radio astronomer from the National Astronomical Observatory of China interested in solar physics and space weather.

\section{Acknowledgements}

The authors thank Kazunari Shibata and Joseph Borovsky for providing leadership to the Task Group 3: How does short-term solar variability affect the geospace environment? The authors also thank CAWSES-II leaders Susan Avery, Alan Rodger, Toshitaka Tsuda, and Joseph Davila for their effort in running a successful SCOSTEP scientific program. We thank P. Mäkelä and Jing Huang for carefully reading the manuscript. We also thank Kazunari Shibata for his comments on the manuscript. The work of NG was supported by NASA's LWS TR\&T program. Portions of this research were performed at the Jet Propulsion Laboratory, California Institute of Technology under contract with NASA. We thank the anonymous referees for the helpful comments.

\section{Author details}

'Solar Physics Laboratory, Code 671, Heliophysics Division, NASA Goddard Space Flight Center, Greenbelt, MD 20771, USA. ${ }^{2}$ Jet Propulsion Laboratory, California Institute of Technology, 4800 Oak Grove Drive, Pasadena, CA 91109, USA. ${ }^{3}$ Key Laboratory of Solar Activity, National Astronomical Observatories, Chinese Academy of Sciences, 20A Datun Road, Chaoyang District, Beijing 100012, China.

Received: 16 December 2014 Accepted: 24 April 2015

Published online: 17 June 2015

\section{References}

Abunina MA, Abunin AA, Belov CACV, Eroshenko EA, Asipenka AS, Oleneva VA, Yanke VG (2013) Relationship between Forbush effect parameters and the heliolongitude of solar sources. Geomagnetism and Aeronomy 53:10-18

Afraimovich EL, Edemskiy IK, Leonovich AS, Leonovich LA, Voeykov SV, Yasyukevich W (2009) MHD nature of night-time MSTIDs excited by the solar terminator. GRL 36:L15106, doi:10.1029/2009GL039803

Ahluwalia HS, Alania MV, Wawrzynczak A, Ygbuhay RC, Fikani MM (2014) May 2005 halo CMEs and galactic cosmic ray flux changes at Earth's orbit. Sol Phys 289:1763-1782

Aguilar-Rodriguez E, Blanco-Cano X, Gopalswamy N (2006) Composition and magnetic structure of interplanetary coronal mass ejections at $1 \mathrm{AU}$. AdSpR 38:522-527

Altrock RC (2014) Forecasting the maxima of solar cycle 24 with coronal Fe xiv emission. SoPh 289:623-629

Allen J, Sauer H, Frank L, Reiff P (1989) Effects of the march 1989 solar activity, EOS trans. AGU 70:1479

Alves MV, Echer E, Gonzalez WD (2011) Geoeffectiveness of solar wind interplanetary magnetic structures. JASTP 73:1380-1384

Akiyama S, Gopalswamy N, Yashiro S, Mäkelä P (2013) A study of coronal holes observed by SoHO/EIT and the Nobeyama Radioheliograph. PASJ 65:15 
Araujo-Pradere EA, Redmon R, Fedrizzi M, Viereck R, Fuller-Rowell TJ (2011) Some characteristics of the ionospheric behavior during the solar cycle 23-24 minimum. SoPh 274:439-456

Arge CN, Henney CJ, Hernandez IG, Toussaint WA, Koller J, Godinez HC (2013) Modeling the corona and solar wind using ADAPT maps that include far-side observations. AIPC 1539:11-14

Arunbabu KP, Antia HM, Dugad SR, Gupta SK, Hayashi Y, Kawakami S, Mohanty PK, Nonaka T, Oshima A, Subramanian P (2013) High-rigidity Forbush decreases: due to CMEs or shocks? A\&A 555:A139

Asai A, Hara H, Watanabe T, Imada S (2012a) Flare onset observed with hinode in the 2006 December 13 flare. In: Sekii T, Watanabe T, Sakurai T (eds) Hinode-3: the $3 \mathrm{rd}$ hinode science meeting, proceedings of the conference held 1-4 December 2009 at Hitotsubashi Memorial Hall, Tokyo, Japan. ASP conference series, vol 454. Astronomical Society of the Pacific, San Francisco, p 303

Asai A, Ishii TT, Isobe H, Kitai R, Ichimoto K, UeNo S, Nagata S, Morita S, Nishida K, Shiota D, Oi A, Akioka M, Shibata K (2012b) First simultaneous observation of an Ha Moreton wave, EUV wave, and filament/prominence oscillations. Astrophys J 745:L18

Asai A, Kiyohara J, Takasaki H, Narukage N, Yokoyama T, Masuda S, Shimojo M, Nakajima H (2013) Temporal and spatial analyses of spectral indices of nonthermal emissions derived from hard X-rays and microwaves. Astrophys J 763:87

Aschwanden MJ (2011) Solar stereoscopy and tomography. LRSP 8:5

Aschwanden MJ, Wülser J-P, Nitta NV, Lemen JR, Freeland S, Thompson WT (2014) STEREO/ Extreme Ultraviolet Imager (EUVI) event catalog 2006-2012. Sol Phys 289:919-938

Baker DN, Li X, Pulkkinen A, Ngwira CM, Mays LL, Galvin AB, Simunac KDC (2013) A major solar eruptive event in July 2012: defining extreme space weather scenarios. Space Weather 11:585-591

Basu S (2013) The peculiar solar cycle 24 - where do we stand? JPhCS 440:012001

Bein BM, Berkebile-Stoiser S, Veronig AM, Temmer M, Muhr N, Kienreich I, Utz D, Vrsnak B (2011) Impulsive acceleration of coronal mass ejections. I. Statistics and coronal mass ejection source region characteristics. Astrophys J 738:191

Belov AA, Abunina M, Eroshenko E, Oleneva V, Yanke V, Papaioannou A, Mavromichalaki H, Gopalswamy N, Yashiro S (2014) Coronal mass ejections and Non-recurrent Forbush decreases. Solar Phys 289:3949-3960

Bemporad A, Mancuso S (2011) Identification of super- and subcritical regions in shocks driven by coronal mass ejections. Astrophys J 739:L64

Bemporad A, Mancuso S (2013) Super- and sub-critical regions in shocks driven by radio-loud and radio-quiet CMEs. JAdR 4:287-291

Bemporad A, Susino R, Lapenta G (2014) Plasma physical parameters along coronal-mass-ejection-driven shocks. I. Ultraviolet and white-light observations. Astrophys J 784:102

Blanc M, Richmond AD (1980) The ionospheric disturbance dynamo. JGRA 85:1669

Borovsky JE (2013) Physical improvements to the solar wind reconnection control function for the Earth's magnetosphere. JGRA 118:2113-2121

Borovsky JE, Denton MH (2013) The differences between storms driven by helmet streamer CIRs and storms driven by pseudostreamer CIRs. JGRA 118:5506-5521

Burgess D, Scholer M (2013) Microphysics of quasi-parallel shocks in collisionless plasmas. SSRv 178:513-533

Burlaga L, Sittler E, Mariani F, Schwenn R (1981) Magnetic loop behind an interplanetary shock - Voyager, Helios, and IMP 8 observations. JGR 86:6673

Burlaga LF, Klein L, Sheeley NR Jr, Michels DJ, Howard RA, Koomen MJ, Schwenn $\mathrm{R}$, Rosenbauer H (1982) A magnetic cloud and a coronal mass ejection. GRL 9:1317-1320

Charbonneau P (2010) Dynamo models of the solar cycle. LRSP 7:3

Chatterjee P, Fan Y (2013) Simulation of homologous and cannibalistic coronal mass ejections produced by the emergence of a twisted flux rope into the solar corona. Astrophys J 778:L8

Chen J (2012) Structure of non-force-free magnetic flux ropes in an ambient medium. Astrophys J 761:179

Chen J, Howard RA, Brueckner GE, Santoro R, Krall J, Paswaters SE, StCyr OC, Schwenn R, Lamy P, Simnett GM (1997) Evidence of an erupting magnetic flux rope: LASCO coronal mass ejection of 1997 april 13. Astrophys J 490:L191-L194

Chen PF (2011) Coronal mass ejections: models and their observational basis. LRSP 8:1

Cheng X, Zhang J, Ding MD, Liu Y, Poomvises W (2013) The driver of coronal mass ejections in the low corona: a flux rope. Astrophys J 763:43
Cho KS, Park SH, Marubashi K, Gopalswamy N, Akiyama S, Yashiro S, Kim R-S, Lim E-K (2013) Comparison of helicity signs in interplanetary CMEs and their solar source regions. SoPh 284:105-127

Cid C, Cremades H, Aran A, Mandrini C, Sanahuja B, Schmieder B, Menvielle M, Rodriguez L, Saiz E, Cerrato Y, Dasso S, Jacobs C, Lathuillere C, Zhukov A (2012) Can a halo CME from the limb be geoeffective? JGRA 117:11102

Chertok IM, Grechnev W, Belov AV, Abunin AA (2013) Magnetic Flux of EUV Arcade and Dimming Regions as a Relevant Parameter for Early Diagnostics of Solar Eruptions - Sources of Non-recurrent Geomagnetic Storms and Forbush Decreases. SoPh 282:175-199.

Cliver EW, Feynman J, Garrett HB (1990) An estimate of the maximum speed of the solar wind, 1938-1989. JGR 95:17103-17112

Cliver EW, Ling AG, Belov A, Yashiro S (2012) Size distributions of solar flares and solar energetic particle events. Astrophys J 756:LL29

Cliver EW, Dietrich WF (2013) The 1859 space weather event revisited: limits of extreme activity. JSWSC 3:A31

Colaninno RC, Vourlidas A, Wu CC (2013) Quantitative comparison of methods for predicting the arrival of coronal mass ejections at Earth based on multiview imaging. JGR 118:6866-6879

Cranmer SR (2009) Coronal holes. LRSP 6:3

Dalla S, Agueda N (2010) Role of latitude of source region in solar energetic particle events. In: Maksimovic M, Issautier K, Meyer-Vernet N et al (eds) AIP Conf. Proc. 1216. AIP, Melville, NY, p 613

Davis CJ, Kennedy J, Davis JA (2010) Assessing the accuracy of CME speed and trajectory estimates from STEREO observations through a comparison of independent methods. Solar Phys 263:209-222

Dasso S, Démoulin P, Gulisano AM (2012) Magnetic clouds along the solar cycle: expansion and magnetic helicity. IAUS 286:139-148

Démoulin P (2014) Evolution of interplanetary coronal mass ejections and magnetic clouds in the heliosphere. IAUS 300:245-254

Denton MH, Borovsky JE, Skoug RM, Thomsen MF, Lavraud B, Henderson M, McPherron RL, Zhang JC, Liemohn MW (2006) Geomagnetic storms driven by ICME- and CIR-dominated solar wind. JGRA 111:7

Denton MH, Borovsky JE (2012) Magnetosphere response to high-speed solar wind streams: a comparison of weak and strong driving and the importance of extended periods of fast solar wind. JGRA 117:A00L05

De Toma G (2011) Evolution of coronal holes and implications for high speed solar wind during the minimum between cycles 23 and 24. SoPh 274:195

Downs C, Roussev II, van der Holst B, Lugaz N, Sokolov IV (2012) Understanding SDO/AIA observations of the 2010 june 13 EUV wave event: direct insight from a global thermodynamic MHD simulation. Astrophys J 750:134

Dresing N, Gómez-Herrero R, Klassen A, Heber B, Kartavykh Y, Dröge W (2012) The large longitudinal spread of solar energetic particles during the 17 January 2010 solar event. SoPh 281:281-300

Du AM, Tsurutani BT, Sun W (2011) Solar wind energy input during prolonged, intense northward interplanetary magnetic fields: a new coupling function. JGRA 116:A12215

Dumbovic M, Vrsnak B, Calogovic J, Zupan R (2012) Cosmic ray modulation by different types of solar wind disturbances. A\&A 538:A28

Dungey JW (1961) Interplanetary magnetic field and the auroral zones. PRL 6:47

Echer E, Gonzalez WD, Tsurutani BT, Gonzalez ALC (2008a) Interplanetary conditions causing intense geomagnetic storms (Dst $\leq-100 \mathrm{nT}$ ) during solar cycle 23 (1996-2006). JGRA 113:A05221

Echer E, Gonzalez WD, Tsurutani BT (2008b) Interplanetary conditions leading to superintense geomagnetic storms (Dst $\leq-250 \mathrm{nT}$ ) during solar cycle 23. GRL 35:L06S03

Echer E, Tsurutani BT, Guarnieri FL (2010) Interplanetary origins of November 2004 superstorms. JASTP 72:280

Echer E, Tsurutani BT, Gonzalez WD, Kozyra JU (2011a) High speed stream properties and related geomagnetic activity during the whole heliosphere interval (WHI): 20 march to 16 April 2008. Sol Phys 274:303-320

Echer E, Gonzalez WD, Tsurutani BT (2011 b) Statistical studies of geomagnetic storms with peak Dst $\leq-50$ nT from 1957 to 2008. JASTP 73:1454-1459

Echer E, Tsurutani BT, Guarnieri FL, Kozyra JU (2011c) Interplanetary fast forward shocks and their geomagnetic effects: CAWSES events. J Atmos Sol-Terr Phy 73:1330-1338

Echer E, Tsurutani BT, Gonzalez WD (2012) Extremely low geomagnetic activity during the recent deep solar cycle minimum. IAUS 286:200-209

Echer E, Tsurutani BT, Gonzalez WD (2013) Interplanetary origins of moderate $(-100 \mathrm{nT}<$ Dst $\leq-50 \mathrm{nT})$ geomagnetic storms during solar cycle 23 (1996-2008). JGR 118:385 
Emslie AG, Dennis BR, Shih AY, Chamberlin PC, Mewaldt RA, Moore CS, Share GH, Vourlidas A, Welsch BT (2012) Global energetics of thirty-eight large solar eruptive events. Astrophys J 759:71

Farrugia CJ, Erkaev NV, Jordanova VK, Lugaz N, Sandholt PE, Mühlbachler S, Torbert RB (2013) Features of the interaction of interplanetary coronal mass ejections/magnetic clouds with the Earth's magnetosphere. JASTP 99:14-26

Forbes TG, Linker JA, Chen J, Cid C, Kóta J, Lee MA, Mann G, Mikic Z, Potgieter MS, Schmidt JM, Siscoe GL, Vainio R, Antiochos SK, Riley P (2006) CME theory and models. SSRv 123:251-302

Fushishita A, Kuwabara T, Kato C, Yasue S, Bieber JW, Evenson P, Da Silva MR, Dal Lago A, Schuch NJ, Tokumaru M, Duldig ML, Humble JE, Sabbah I, Al Jassar HK, Sharma MM, Munakata K (2010) Precursors of the Forbush decrease on 2006 December 14 observed with the global muon detector network (GMDN). Astrophys J 715:1239-1247

Gallagher PT, Long DM (2011) Large-scale bright fronts in the solar corona: a review of "EIT waves". Space Sci Rev 158:365-396

Gold T (1962) Magnetic storms. SSRv 1:100

Goldstein H (1983), On the field configuration in magnetic clouds. In Solar Wind Five, edited by M. Neugebauer, NASA Conf. Publ., CP-2280, 731-733

Gonzalez WD, Joselyn JA, Kamide Y, Kroehl HW, Rostoker G, Tsurutani BT, Vasyliunas V (1994) What is a geomagnetic storm? JGRA 99:5771

Gonzalez WD, Gonzalez ALC, Dal Lago A, Tsurutani BT, Arballo JK, Lakhina GS, Buti B, Ho GM, Wu ST (1998) Magnetic cloud field intensities and solar wind velocities. GRL 25:963

Gopalswamy N (2006a) Properties of interplanetary coronal mass ejections. SSRV $124: 145-168$

Gopalswamy N (2006b) Coronal mass ejections and type II radio bursts. In Solar Eruptions and Energetic Particles - Editors: N. Gopalswamy, R. Mewaldt, J. Torsti; Washington DC, Am. Geophy. Union Press: Geophysical Monograph Series - Volume 165 p. 207

Gopalswamy N (2008) Solar connections of geoeffective magnetic structures. JASTP 70:2078-2100

Gopalswamy N (2009) Coronal mass ejections and space weather. In: Tsuda T, Fujii R, Shibata K, Geller MA (eds) Climate and Weather of the Sun-Earth System (CAWSES): Selected Papers from the 2007 Kyoto Symposium. Terrapub, Tokyo, p 77

Gopalswamy, N. (2010) The CME link to geomagnetic storms. Solar and Stellar Variability: Impact on Earth and Planets, Proceedings of the International Astronomical Union, IAU Symposium; Paris, International Astronomical Union: Volume 264, p. 326-335

Gopalswamy N (2011) Coronal mass ejections and solar radio emissions. In Proceedings of the 7th International Workshop on Planetary, Solar and Heliospheric Radio Emissions (PRE VII); Graz, Austrian Academy of Sciences Press: p. 325-342

Gopalswamy N (2012) Energetic particle and other space weather events of solar cycle 24. AIPC 1500:14-19

Gopalswamy N, Shibasaki K, Salem M (2000) Microwave enhancement in coronal holes: statistical properties. JApA 21:413

Gopalswamy N, Yashiro S, Kaiser ML, Howard RA, Bougeret J-L (2001a) Radio signatures of coronal mass ejection interaction: coronal mass ejection cannibalism? Astrophys J 548:L91-L94

Gopalswamy N, Lara A, Kaiser ML, Bougeret J-L (2001b) Near-Sun and near-earth manifestations of solar eruptions. JGR 106:25261-25278

Gopalswamy N, Aguilar-Rodriguez E, Yashiro S, Nunes S, Kaiser ML, Howard RA (2005a) Type II radio bursts and energetic solar eruptions. JGRA 110:A12S07

Gopalswamy N, Yashiro S, Michalek G, Xie H, Lepping RP, Howard RA (2005b) Solar source of the largest geomagnetic storm of cycle 23. GRL 32:L12S09

Gopalswamy N, Yashiro S, Liu Y, Michalek G, Vourlidas A, Kaiser ML, Howard RA (2005c) Coronal mass ejections and other extreme characteristics of the 2003 October-November solar eruptions. JGRA 110:A09S15

Gopalswamy N, Yashiro S, Xie H, Akiyama S, Aguilar-Rodriguez E, Kaiser ML, Howard RA, Bougeret JL (2008a) Radio-quiet fast and wide coronal mass ejections. Astrophys J 674:560-569

Gopalswamy N, Akiyama S, Yashiro S, Michalek G, Lepping RP (2008b) Solar sources and geospace consequences of interplanetary magnetic clouds observed during solar cycle 23. JASTP 70:245-253

Gopalswamy N, Yashiro S, Michalek G, Stenborg G, Vourlidas A, Freeland S, Howard RA (2009a) The SOHO/LASCO CME catalog. Earth Moon Planets 104:295-313

Gopalswamy N, Thompson WT, Davila JM, Kaiser ML, Yashiro S, Mäkelä P, Michalek G, Bougeret JL, Howard RA (2009b) Relation between type II bursts and CMEs inferred from STEREO observations. SoPh 259:227-254
Gopalswamy N, Yashiro S, Temmer M, Davila J, Thompson WT, Jones S, McAteer RTJ, Wuelser JP, Freeland S, Howard RA (2009c) EUV wave reflection from a coronal hole. Astrophys J 691:L123-L127

Gopalswamy N, Mäkelä P, Xie H, Akiyama S, Yashiro S (2009d) CME interactions with coronal holes and their interplanetary consequences. JGRA 114:A00A22

Gopalswamy N, Yashiro S, Michalek G, Xie H, Mäkelä P, Vourlidas A, Howard RA (2010a) A catalog of halo coronal mass ejections from SOHO. Sun and Geosphere 5:7-16

Gopalswamy N, Akiyama S, Yashiro S, Mäkelä P (2010b) Coronal mass ejections from sunspot and non-sunspot regions. In Magnetic Coupling between the Interior and Atmosphere of the Sun, Astrophysics and Space Science Proceedings, eds. by S. S. Hasan and R. J. Rutten; Berlin Heidelberg, Springer-Verlag Goldstein 1983 Pasadena, CA: Jet Propulsion Laboratory p. 289

Gopalswamy N, Xie H, Mäkelä P, Akiyama S, Yashiro S, Kaiser ML, et al (2010c) Interplanetary shocks lacking type II radio bursts. Astrophys J 710:1111

Gopalswamy N, Mäkelä P, Xie H, Akiyama S, Yashiro S (2010d) Solar sources of "driverless" interplanetary shocks. Twelfth International Solar Wind Conference, AIP Conference Proceedings; Melville NY, AIP Publishing LLC: Volume 1216, p. 452

Gopalswamy N, Yashiro S (2011) The strength and radial profile of the coronal magnetic field from the standoff distance of a coronal mass ejection-driven shock. Astrophys J 736:LL17

Gopalswamy N, Yashiro S, Mäkelä P, Michalek G, Shibasaki K, Hathaway DH (2012a) Behavior of solar cycles 23 and 24 revealed by microwave observations. Astrophys J 750:LL42

Gopalswamy N, Nitta N, Akiyama S, Mäkelä P, Yashiro S (2012b) Coronal magnetic field measurement from EUV images made by the Solar Dynamics Observatory. Astrophys J 744:72

Gopalswamy N, Mäkelä P, Akiyama S, Yashiro S, Xie H, MacDowall RJ, Kaiser ML (2012C) Radio-loud CMEs from the disk center lacking shocks at 1 AU. JGRA 117:A08106

Gopalswamy N, Xie H, Yashiro S, Akiyama S, Mäkelä P, Usoskin IG (2012d) Properties of ground level enhancement events and the associated solar eruptions during solar cycle 23. SSRv 171:23-60

Gopalswamy N, Xie H, Akiyama S, Yashiro S, Usoskin IG, Davila JM (2013a) The first ground level enhancement event of solar cycle 24: direct observation of shock formation and particle release heights. Astrophys J 765:L30

Gopalswamy N, Xie H, Mäkelä P, Yashiro S, Akiyama S, Uddin W, et al (2013b) Height of shock formation in the solar corona inferred from observations of type II radio bursts and coronal mass ejections. AdSpR 51:1981-1989

Gopalswamy N, Mäkelä P, Akiyama S, Xie H, Yashiro S, Reinard AA (2013c) The solar connection of enhanced heavy lon charge states in the interplanetary medium: implications for the flux-rope structure of CMEs. SoPh 284:17-46

Gopalswamy N, Nieves-Chinchilla T, Hidalgo M, Zhang J, Riley P, van DrielGesztelyi L, Mandrini CH (2013d) Preface. SoPh 284:1-4

Gopalswamy N, Mäkelä P, Xie H, Yashiro S (2013e) Testing the empirical shock arrival model using quadrature observations. SpWea 11:661-669

Gopalswamy N, Akiyama S, Yashiro S, Xie H, Mäkelä P, Michalek G (2014a) Anomalous expansion of coronal mass ejections during solar cycle 24 and its space weather implications. GRL 41:2673-2680

Gopalswamy N, Xie H, Akiyama S, Mäkelä P, Yashiro S (2014b) Major solar eruptions and high-energy particle events during solar cycle 24. Earth Planets space 66:104-118

Gopalswamy N, Mäkelä P (2014) Latitudinal connectivity of ground level enhancement events. ASPC 484:63

Gopalswamy N, Xie H, Akiyama S, Mäkelä P, Yashiro S, Michalek G (2015) The peculiar behavior of halo coronal mass ejections in solar cycle 24. Astrophys U in press

Gosling JT (1990) Coronal mass ejections and magnetic flux ropes in interplanetary space. GMS 58:343-364

Gosling JT (1996) Corotating and transient solar wind flows in three dimensions. ARA\&A 34:35-74

Gui B, Shen CL, Wang YM, Ye PZ, Liu JJ, Wang S, Zhao X (2011) Quantitative analysis of CME deflections in the corona. Solar Physics 271:111-139

Hajra R, Tsurutani BT, Gonzalez WD (2013) Solar cycle dependence of high-intensity long-duration continuous AE activity (HILDCAA) events, relativistic electron predictors? JGR 118:5626

Hajra R, Tsurutani BT, Echer E, Gonzalez WD (2014) Relativistic electron acceleration during high-intensity, long-duration, continuous AE activity (HILDCAA) events: solar cycle phase dependences. GRL 41:1876

Hajra R, Tsurutani BT, Echer E, Gonzalez WD, Santolik O ((2015) Relativistic (E > 0.6, > 2.0 and $>4.0 \mathrm{MeV}$ ) electron acceleration at geosynchronous orbit during high-intensity long-duration continuous AE activity (HILDCAA) events. Astrophys J 799:39 
Hariharan K, Ramesh R, Kishore P, Kathiravan C, Gopalswamy N (2014) An estimate of the coronal magnetic field near a solar coronal mass ejection from low-frequency radio observations. Astrophys J 795:14-22

Harra LK, Sterling AC, Gömöry P, Veronig A (2011) Spectroscopic observations of a coronal Moreton wave. Astrophys J 737:L4

Harrison RA, Davies JA, Möstl C, Liu Y, Temmer M, Bisi MM, Eastwood JP, de Koning CA, Nitta N, Rollett T, Farrugia CJ, Forsyth RJ, Jackson BV, Jensen EA, Kilpua EKJ, Odstrcil D, Webb DF (2012) An analysis of the origin and propagation of the multiple coronal mass ejections of 2010 august 1. Astrophys J 750:45

Hathaway DH (2010) The solar cycle. LRSP 7:1

Hollweg JV (2006) The solar wind: then and now, in recurrent magnetic storms: corotating solar wind streams. edited by B. Tsurutani, R. McPherron, W. Gonzalez, G. Lu, J.H.A. Sobral and N. Gopalswamy, AGU; Washington DC, Am. Geophy. Union Press: 167:19

Horne RB (2007) Acceleration of killer electrons. Nat Phys 3:590

Horne RB, Thorne RM (2003) Relativistic electron acceleration and precipitation during resonant interactions with whistler-mode chorus. GRL 30:1527, doi:10.1029/2003GL016973

Howard RA, Michels DJ, Sheeley NR Jr, Koomen MJ (1982) The observation of a coronal transient directed at earth. Astrophys J 263:L101

Huang J, Demoulin P, Pick M, Auchere F, Yan YH, Bouteille A (2011) Initiation and early development of the 2008 April 26 coronal mass ejection. Astrophys J 729:107

Huba JD, Dymond KF, Joyce G, Budzien SA, Thonnard SE, Fedder JA, McCoy RP (2002) Comparison of O+ density from ARGOS LORAAS data analysis and SAMI2 model results. GRL 29:6-1, doi:10.1029/2001GL013089

Hundhausen AJ (1987) Proc. Sixth international solar wind conference. In: Pizzo VJ, Holzer TE, Sime DG (eds) High altitude observatory, vol 1. NCAR, Boulder, Colorado, p 181

Isavnin A, Vourlidas A, Kilpua EKJ (2014) Three-dimensional evolution of flux-rope CMEs and its relation to the local orientation of the heliospheric current sheet. SoPh 289:2141-2156

Jackson BV, Clover JM, Hick PP, Buffington A, Bisi MM, Tokumaru M (2013) Inclusion of real-time in-situ measurements into the UCSD time-dependent tomography and its use as a forecast algorithm. SoPh 285:151-165

Janvier M, Demoulin P, Dasso S (2013) Global axis shape of magnetic clouds deduced from the distribution of their local axis orientation. A\&A 556:AA50

Javaraiah J (2015) Long-term variations in the north-south asymmetry of solar activity and solar cycle prediction, III: Prediction for the amplitude of solar cycle 25. NewA 34:54-64

Jordanova VK, Welling DT, Zaharia SG, Chen L, Thorne RM (2012) Modeling ring current ion and electron dynamics and plasma instabilities during a high-speed stream driven storm. JGRA 117:A00L08

Jiang J, Cameron RH, Schmitt D, Isik E (2013a) Modeling solar cycles 15 to 21 using a flux transport dynamo. A\&A 553:A128

Jiang J, Cameron RH, Schmitt D, Schüssler M (2013b) Can surface flux transport account for the weak polar field in cycle 23? SSRv 176:289-298

Joshi NC, Uddin W, Srivastava AK, Chandra R, Gopalswamy N, Manoharan PK, Aschwanden M, Choudhary DP, Jain R, Nitta NV, Xie H, Yashiro S, Akiyama S, Mäkelä P, Kayshap P, Awasthi AK, Dwivedi VC, Mahalakshmi K (2013) A multi-wavelength study of eruptive events on January 23, 2012 associated with a major solar energetic particle event. AdSR 52:1

Kahler SW, Arge CN, Akiyama S, Gopalswamy N (2014) Do solar coronal holes affect the properties of solar energetic particle events? SoPh 289:657-673

Kahler SW, Vourlidas A (2014) Do interacting coronal mass ejections play a role in solar energetic particle events? Astrophys J 784:47

Kataoka R (2013) Probability of occurrence of extreme magnetic storms. SpWea 11:214-218

Kay C, Opher M, Evans RM (2013) Forecasting a coronal mass ejection's altered trajectory: ForeCAT. Astrophys J 775:5

Kazachenko MD, Canfield RC, Longcope DW, Qiu J (2012) Predictions of energy and helicity in four major eruptive solar flares. SoPh 277:165-183

Keesee AM, Elfritz JG, Fok MC, McComas DJ, Scime EE (2014) Superposed epoch analyses of ion temperatures during CME- and CIR/HSS-driven storms. JASTP 115:67-78

Kelley MC, Makela JJ, Chau JL, Nicolls MJ (2003) Penetration of the solar wind electric field into the magnetosphere/ionosphere system. GRL 30:1158, doi:10.1029/2002GL016321

Kennel CF, Edmiston JP, Scarf FL, Coroniti FV, Russell CT, Smith EJ, Tsurutani BT, Scudder JD, Feldman WC, Anderson RR, Moser FS, Temerin M (1984a) Structure of the November 12, 1978 quasi-parallel interplanetary shock. JGR 89:5436
Kennel CF, Scarf FL, Coroniti FV, Russell CT, Wenzel KP, Sanderson TR, Van Nes P, Feldman WC, Parks GK, Smith EJ, Tsurutani BT, Mozer FS, Temerin M, Anderson RR, Scudder JD, Scholer M (1984b), JGR 89:5419

Kennel CF, Edmiston JP, Hada T (1985) A quarter century of collisionless shock research. In: Stone R, Tsurutani BT (eds) Collisionless shocks in the heliosphere: a tutorial review, vol 34. Geoph. Mon. Ser., AGU, Wash. DC, p 1

Kienreich IW, Muhr N, Veronig AM, Berghmans D, De Groof A, Temmer M, Vrsnak B, Seaton DB (2013) Solar TErrestrial Relations Observatory-A (STEREO-A) and PRoject for On-Board Autonomy 2 (PROBA2) quadrature observations of reflections of three EUV waves from a coronal hole. SoPh 286:201-21

Kilpua EKJ, Luhmann JG, Jian LK, Russell CT, Li Y (2014) Why have geomagnetic storms been so weak during the recent solar minimum and the rising phase of cycle 24? JASTP 107:12-19

Kim R-S, Gopalswamy N, Cho K-S, Moon Y-J, Yashiro S (2013) Propagation characteristics of CMEs associated with magnetic clouds and ejecta. Sol Phys 284:77-88

Kim R-S, Gopalswamy N, Moon Y-J, Cho K-S, Yashiro S (2012) Magnetic field strength in the upper solar corona using white-light shock structures surrounding coronal mass ejections. Astrophys J 746:118

Kleimann J (2012) 4 pi models of CMEs and ICMEs (invited review). SoPh 281:353-367

Koomen M, Howard R, Hansen R, Hansen S (1974) The coronal transient of 16 June 1972. SoPh 34:447

Kovaltsov GA, Usoskin IG (2014) Occurrence probability of large solar energetic particle events: assessment from data on cosmogenic radionuclides in lunar rocks. SoPh 289:211-220

Kozarev KA, Korreck KE, Lobzin W, Weber MA, Schwadron NA (2011) Off-limb solar coronal wavefronts from SDO/AIA extreme-ultraviolet observations-implications for particle production. Astrophys J 733:LL25

Kozyra JU, Liemohn MW, Cattell C, De Zeeuw D, Escoubet CP, Evans DS, Fang X, Fok M-C, Frey HU, Gonzalez WD, Hairston M, Heelis R, Lu G, Manchester WB IV, Mende S, Paxton LJ, Rastaetter L, Ridley A, Sandanger M, Soraas F, Sotirelis T, Thomsen MW, Tsurutani BT, Verkhoglyadova O (2014) Solar filament impact on 21 January 2005: geospace consequences. JGR 119:5401, doi:10.1002/2013JA019748

Kudela K (2013) Space weather near Earth and energetic particles: selected results. JPhCS 409:012017

Kuin PN, Martens PCH (1986) A dynamic model of filament eruptions and two ribbon flares. NASA CP-2442:241-245

Kusano K, Bamba Y, Yamamoto TT, lida Y, Toriumi S, Asai A (2012) Magnetic field structures triggering solar flares and coronal mass ejections. Astrophys J 760:31-39

Lamy P, Barlyaeva T, Llebaria A, Floyd O (2014) Comparing the solar minima of cycles 22/23 and 23/24: the view from LASCO white light coronal images. JGRA 119:47-58

Lario D, Decker RB (2002) The energetic storm particle event of October 20, 1989. GRL 29:1393

Lario D, Kallenrode M-B, Decker RB, Roelof EC, Krimigis SM, Aran A, Sanahuja B (2006) Radial and longitudinal dependence of solar 4-13 MeV and 27-37 MeV proton peak intensities and fluences: Helios and IMP 8 observations. Astrophys J 653:1531-1544

Lario DA, Aran R, Gomez-Herrero D, Dresing N, Heber B, Ho GC, Decker RB, Roelof EC (2013) Longitudinal and radial dependence of solar energetic particle peak intensities: STEREO, ACE, SOHO, GOES and MESSENGER observations. Astrophys J 767:41-58

Lario D, Roelof EC, Decker RB (2014) Longitudinal dependence of SEP peak intensities as evidence of CME-driven shock particle acceleration. ASPC 484:98

Leamon RJ, Canfield RC, Jones SL, Lambkin K, Lundberg BJ, Pevtsov AA (2004) Helicity of magnetic clouds and their associated active regions. JGRA 109:A05106

Lean JL, McDonald SE, Huba JD, Emmert JT, Drob DP, Siefring CL (2014) Geospace variability during the 2008-2009 whole heliosphere intervals. JGRA 119:3755-3776

Lee MA (1983). Coupled hydromagnetic wave excitation and ion acceleration at interplanetary traveling shocks, JGR, 79, 489.

Lei J, Thayer JP, Forbes JM, Wu Q, She C, Wan W, Wang W (2008) lonosphere response to solar wind high-speed streams. GRL 35:L19105, doi:10.1029/2008GL035208

Lei J, Thayer JP, Wang W, McPherron RL (2011) Impact of CIR storms on thermosphere density variability during the solar minimum of 2008. SoPh 274:427-437

Li LP, Zhang J (2013a) Eruptions of two flux ropes observed by SDO and STEREO. A\&A 552:11 
Li T, Zhang J (2013b) Fine-scale structures of flux ropes tracked by erupting material. Astrophys J 770:25

Li T, Zhang J (2013c) Homologous flux ropes observed by the solar dynamics observatory atmospheric imaging assembly. Astrophys J 778:L29

Liemohn MW, Katus R (2012) Is the storm time response of the inner magnetospheric hot ions universally similar or driver dependent? JGRA 117:A00L03

Lionello R, Downs C, Linker JA, Török T, Riley P, Mikić Z (2013) Magnetohydrodynamic simulations of interplanetary coronal mass ejections. Astrophys J 777:76

Liu W, Ofman L (2014) Advances in observing various coronal EUV waves in the SDO era and their seismological applications (invited review). SoPh 289:3233-3277

Liu YD, Luhmann JG, Lugaz N, Möstl C, Davies JA, Bale SD, Lin RP (2013) On Sun-to-Earth propagation of coronal mass ejections. Astrophys J 765:45-59

Liu YD, Yang Z, Wang R, Luhmann JG, Richardson JD, Lugaz N (2014a) Sun-to-Earth characteristics of two coronal mass ejections interacting near $1 \mathrm{AU}$ : formation of a complex ejecta and generation of a two-step geomagnetic storm. Astrophys $J$ 793:L41

Liu YD, Luhmann JG, Kajdic P, Kilpua EK, Lugaz N, Nitta NV, et al (2014b) Observations of an extreme storm in interplanetary space caused by successive coronal mass ejections. NatCo 5:3841

Livingston W, Harvey JW, Malanushenko OV, Webster L (2006) Sunspots with the strongest magnetic fields. SoPh 239:41-68

Lockwood M (2013) Reconstruction and prediction of variations in the open solar magnetic flux and interplanetary conditions. LRSP 10:4

Lockwood M, Owens MJ, Barnard L, Davis CJ, Steinhilber F (2011) The persistence of solar activity indicators and the descent of the Sun into Maunder minimum conditions. GRL 38:L22105

Long DM, Williams DR, Régnier S, Harra LK (2013) Measuring the magnetic-field strength of the quiet solar corona using "EIT waves". Sol Phys 288:567-583

Long DM, Gallagher PT, McAteer RTJ, Bloomfield DS (2008) The kinematics of a globally propagating disturbance in the solar corona. Astrophys I 680:L81-L84

Lugaz N, Farrugia CJ, Davies JA, Mostl C, Davis CJ, Roussev II, Temmer M (2012) The deflection of the two interacting coronal mass ejections of 2010 May 23-24 as revealed by combined in situ measurements and heliospheric imaging. Astrophys J 759:68

Lugaz N, Farrugia CJ, Manchester WB IV, Schwadron N (2013) The interaction of Two coronal mass ejections: influence of relative orientation. Astrophys J $778: 20$

Ma S, Raymond JC, Golub LJ, Chen H, Grigis P, Testa P, Long D (2011) Observations and interpretation of a low coronal shock wave observed in the EUV by the SDO/AIA. Astrophys J 738:160

Maehara H, Shibayama T, Notsu S, Notsu Y, Nagao T, Kusaba S, Honda S, Nogami D, Shibata K (2012) Superflares on solar-type stars. Natur 485:478-481

Mäkelä P, Gopalswamy N, Akiyama S, Xie H, Yashiro S (2011) Energetic storm particle events in coronal mass ejection-driven shocks. JGRA 116:A08101

Mäkelä P, Gopalswamy N, Xie H, Mohamed AA, Akiyama S, Yashiro S (2013) Coronal hole influence on the observed structure of interplanetary CMES. SoPh 284:59-75

Maloney SA, Gallagher PT (2011) STEREO direct imaging of a coronal mass ejection driven shock to $0.5 \mathrm{AU}$. Astrophys J 736:LL5

Mann G, Klassen A, Aurass H, Classen H-T (2003) Formation and development of shock waves in the solar corona and the near-Sun interplanetary space. A\&A 400:329-336

Manoharan PK (2010) Ooty interplanetary scintillation - remote-sensing observations and analysis of coronal mass ejections in the heliosphere. SoPh 265:137-157

Mannucci AJ, Tsurutani BT, lijima BA, Komjathy A, Saito A, Gonzalez WD, Guarnieri FL, Kozyra JU, Skoug R (2005) Dayside global ionospheric response to the major interplanetary events of October 29-30, 2003 "Halloween storms". GRL32:L12S02, doi:10.1029/2004GL021467

Mannucci AJ, Tsurutani BT, Abdu MA, Gonzalez WD, Komjathy A, Echer E, lijima BA, Crowley G, Anderson D (2008) Superposed epoch analysis of the dayside ionospheric response to four intense geomagnetic storms, JGR 113:A00A02, doi:10.1029/2007JA012732

Mannucci AJ, Tsurutani BT, Solomon SC, Verkhoglyadova OP, Thayer JP (2012) How do coronal hole storms affect the upper atmosphere? EOS 93:77-79

Mannucci AJ, Crowley G, Tsurutani BT, Verkhoglyadova OP, Komjathy A, Stephens P (2014) Interplanetary magnetic field by control of prompt total electron content increases during superstorms. JASTP 115:7-16
Maričić D, Vršnak B, Dumbović M, Žic T, Roša D, Hržina D, Lulić S, Romštajn I, Bušić I, Salamon K, Temmer M, Rollett T, Veronig A, Bostanjyan N, Chilingarian A, Mailyan B, Arakelyan K, Hovhannisyan A, Mujić N (2014) Kinematics of interacting ICMEs and related Forbush decrease: case study. Sol Phys 289:351-368

Marubashi K (2000) Physics of interplanetary magnetic flux ropes: toward prediction of geomagnetic storms. AdSpR 26:55-66

Marubashi K, Akiyama S, Yashiro S, Gopalswamy N, Cho KS (2015) Geometrical relationship between interplanetary flux ropes and their solar sources. SoPh, 290:1371-1397.

McComas DJ, Ebert RW, Elliott HA, Goldstein BE, Gosling JT, Schwadron NA, Skoug A (2008) Weaker solar wind from the polar coronal holes and the whole Sun. GRL 35:18103

McComas DJ, Angold N, Elliott HA, Livadiotis G, Schwadron NA, Skoug RM, Smith CW (2013) Weakest solar wind of the space Age and the current "mini" solar maximum. Astrophys J 779:2

Meredith NP, Cain M, Horne RB, Thorne RM, Summers D, Anderson RR (2003) Evidence for chorus-driven electron acceleration to relativistic energies from a survey of geomagnetically disturbed periods. JGR 108:1248, doi:10.1029/2002JA009764

Meredith NP, Horne RB, Anderson RR (2001) Substorm dependence of chorus amplitudes: implication for the acceleration of electrons to relativistic energies. JGR 106:13165, doi:10.1029/2000JA900146

Mewaldt RA, Looper MD, Cohen CMS, Haggerty DK, Labrador AW, Leske RA, Mason GM, Mazur JE, von Rosenvinge TT (2012) Energy spectra, composition, and other properties of ground-level events during solar cycle 23. SSRv 171:97-120

Mewaldt RA, Cohen CMS, Mason GM, von Rosenvinge TT, Leske RA, Luhmann JG, Odstrcil D, Vourlidas A (2013) Solar energetic particles and their variability from the Sun and beyond. AIPC 1539:116-121

Millward G, Biesecker D, Pizzo V, de Koning CA (2013) An operational software tool for the analysis of coronagraph images: determining CME parameters for input into the WSA-ENLIL heliospheric model. Space Weather 11:57-68

Michalek G, Gopalswamy N, Xie H (2007) Width of radio-loud and radio-quiet CMEs. SoPh 246:409-414

Miroshnichenko LI, Vashenyuk EV, Pérez-Peraza JA (2013) Solar cosmic rays: 70 years of ground-based observations. Geomagn Aeronomy 53:541-560

Mitra AP (1974) lonospheric effects of solar flares. Springer, New York, 294 pp

Mlynczak M, Martin-Torres FJ, Russell JM, Beaumont K, Jacobson S, Koyzra J et al. (2003) The natural thermostat of nitric oxide emission at $5.3 \mathrm{~nm}$ in the thermosphere observed during solar storms of April 2002. GRL 30:2100, doi:10.1029/2003GL017693

Mohamed AA, Gopalswamy N, Yashiro S, Akiyama S, Mäkelä P, Xie H, Jung H (2012) The relation between coronal holes and coronal mass ejections during the rise, maximum, and declining phases of solar cycle 23. JGRA 117:A01103

Mordvinov AV, Yazev SA (2014) Reversals of the Sun's polar magnetic fields in relation to activity complexes and coronal holes. SoPh 289:1971-1981

Möstl C, Davies JA (2013) Speeds and arrival times of solar transients approximated by self-similar expanding circular fronts. SoPh 285:411-423

Möstl C, Amla K, Hall JR, Liewer PC, De Jong EM, Colaninno RC, Veronig AM, Rollett T, Temmer M, Peinhart V, Davies JA, Lugaz N, Liu YD, Farrugia CJ, Luhmann JG, Vršnak B, Harrison RA, Galvin AB (2014) Connecting speeds, directions and arrival times of 22 coronal mass ejections from the Sun to 1 AU. Astrophys J 789:119-135

Müller D, Marsden RG, St Cyr OC, Gilbert HR (2013) Solar orbiter. Exploring the Sun-heliosphere connection. Sol Phys 285:25-70

Munakata K, Kuwabara T, Yasue S, Kato C, Akahane S, Koyama M, Ohashi Y, Okada A, Aoki T, Mitsui K, Kojima H, Bieber JW (2005) A "loss cone" precursor of an approaching shock observed by a cosmic ray muon hodoscope on October 28, 2003. GRL 32:L03504

Muñoz-Jaramillo A, Dasi-Espuig M, Balmaceda LA, DeLuca EE (2013) Solar cycle propagation, memory, and prediction: insights from a century of magnetic proxies. Astrophys J 767:L25

Nelson GJ, Melrose DB (1985) Type II bursts. In: Solar radiophysics: studies of emission from the sun at metre wavelengths. Cambridge University Press, Cambridge and New York, pp 333-359

Newton HW (1955) The lineage of the great sunspots. VA 1:666-674

Nolte JT, Krieger AS, Timothy AF, Gold RE, Roelof EC, Vaiana G, Lazarus AJ, Sullivan JD, Mclntosh PS (1976) Coronal holes as sources of solar wind. SoPh 46:303-322

Nieves-Chinchilla T, Colaninno R, Vourlidas A, Szabo A, Lepping RP, Boardsen SA, Anderson BJ, Korth H (2012) Remote and in situ observations of an unusual 
Earth-directed coronal mass ejection from multiple viewpoints. JGRA 117:A06106

Nishida A (1968) Coherence of geomagnetic DP2 fluctuations with interplanetary magnetic field variations. JGR 73:5549

Nitta NV, Liu Y, DeRosa ML, Nightingale RW (2012) What are special about ground-level events? Flares, CMEs, active regions and magnetic field connection. SSRV 171:61-83

Nitta NV, Schrijver CJ, Title AM, Liu W (2013a) Large-scale coronal propagating fronts in solar eruptions as observed by the atmospheric imaging assembly on board the Solar Dynamics Observatory - an ensemble study. Astrophys J 776:58

Nitta NV, Aschwanden MJ, Boerner PF, Freeland SL, Lemen JR, Wuelser J-P (2013b) Soft X-ray fluxes of major flares far behind the limb as estimated using STEREO EUV images. SoPh 288:241-254

Nitta NV, Sun X, Hoeksema JT, DeRosa ML (2014) Solar cycle variations of the radio brightness of the solar polar regions as observed by the Nobeyama Radioheliograph. Astrophys J 780:L23

Obridko VN, Shelting BD (2008) On prediction of the strength of the 11-year solar cycle no. 24. SoPh 248:191-202

Olmedo O, Vourlidas A, Zhang J, Cheng X (2012) Secondary waves and/or the "reflection" from and "transmission" through a coronal hole of an extreme ultraviolet wave associated with the 2011 February 15 X2.2 flare observed with SDO/AIA and STEREO/EUVI. Astrophys J 756:143

Ontiveros V, Vourlidas A (2009) Quantitative measurements of coronal mass ejection-driven shocks from LASCO observations. Astrophys J 693:267-275

Owens MJ, Cargill PJ, Pagel C, Siscoe GL, Crooker NU (2005) Characteristic magnetic field and speed properties of interplanetary coronal mass ejections and their sheath regions. JGRA 110:A01105

Owens MJ, Forsyth RJ (2013) The heliospheric magnetic field. LRSP 10:5

Panasenco O, Martin SF, Velli M, Vourlidas A (2013) Origins of rolling, twisting, and non-radial propagation of eruptive solar events. SoPh 287:391-413

Papaioannou A, Souvatzoglou G, Paschalis P, Gerontidou M, Mavromichalaki H (2014) The first ground-level enhancement of solar cycle 24 on 17 May 2012 and its real-time detection. SoPh 289:423-436

Patsourakos S, Vourlidas A (2009) "Extreme ultraviolet waves" are waves: first quadrature observations of an extreme ultraviolet wave from STEREO. Astrophys J 700:L182-L186

Perrault PD, Akasofu SI (1978) A study of geomagnetic storms. Geophys J R Astron Soc 54:547

Pesnell WD (2014) Predicting solar cycle 24 using a geomagnetic precursor pair. SoPh 289:2317-2331

Petrie GJD (2012) Evolution of active and polar photospheric magnetic fields during the rise of cycle 24 compared to previous cycles. SoPh 281:577-598

Petrie GJD (2013) Solar magnetic activity cycles, coronal potential field models and eruption rates. Astrophys J 768:162

Petrovay K (2010) Solar cycle prediction. LSRP 7:6

Poomvises W, Gopalswamy N, Yashiro S, Kwon RY, Olmedo O (2012) Determination of the heliospheric radial magnetic field from the standoff distance of a CME-driven shock observed by the STEREO spacecraft. Astrophys J 758:118

Potgieter M (2013) Solar modulation of cosmic rays. LRSP 10:3

Potgieter MS, Vos EE, Boezio M, De Simone N, Di Felice V, Formato V (2014) Modulation of galactic protons in the heliosphere during the unusual solar minimum of 2006 to 2009. SoPh 289:391-406

Qiu J, Hu Q, Howard TA, Yurchyshyn VB (2007) On the magnetic flux budget in low-corona magnetic reconnection and interplanetary coronal mass ejections. Astrophys J 659:758-772

Reames DV (1999) Particle acceleration at the Sun and in the heliosphere. SSRV 90:413-491

Reames DV (2013) The two sources of solar energetic particles. SSRv 175:53-92

Richardson IG (2013) Geomagnetic activity during the rising phase of solar cycle 24. JSWSC 3:AA08

Riley P, Schatzman C, Cane HV, Richardson IG, Gopalswamy N (2006) On the rates of coronal mass ejections: remote solar and in situ observations. Astrophys J 647:648-653

Riley P, Richardson IG (2013) Using statistical multivariable models to understand the relationship between interplanetary coronal mass ejecta and magnetic flux ropes. SoPh 284:217-233

Rockenbach M, Dal Lago A, Gonzalez WD, Munakata K, Kato C, Kuwabara T, Bieber J, Schuch NJ, Duldig ML, Humble JE, Al Jassar HK, Sharma MM, Sabbah I (2011) Geomagnetic storm's precursors observed from 2001 to 2007 with the Global Muon Detector Network (GMDN). Geophys Res Lett 38:L16108
Rockenbach M, Dal Lago A, Schuch NJ, Munakata K, Kuwabara T, Oliveira AG, Echer E, Braga CR, Mendonça RRS, Kato C, Kozai M, Tokumaru M, Bieber JW, Evenson P, Duldig ML, Humble JE, Al Jassar HK, Sharma MM, Sabbah I (2014) Global muon detector network used for space weather applications. Space Sci Rev 14:1-18

Rouillard AP, Sheeley NR, Tylka A, Vourlidas A, Ng CK, Rakowski C, et al (2012) The longitudinal properties of a solar energetic particle event investigated using modern solar imaging. Astrophys J 752:44

Roussev I, Galsgaard K, Downs C, Lugaz N, Sokolov L, Moise E, Lin J (2012) Explaining fast ejections of plasma and exotic X-ray emission from the solar corona. Nat Phys 8:845

Ruffolo D, Seripienlert A, Tooprakai P, Chuychai P, Matthaeus WH (2013) Squeezing of particle distributions by expanding magnetic turbulence and space weather variability. Astrophys J 779:74

Russell CT, Mulligan T (2002) On the magnetosheath thicknesses of interplanetary coronal mass ejections. P\&SS 50:527-534

Russell CT, Jian LK, Luhmann JG (2013a) How unprecedented a solar minimum was it? JAdR 4:253-258

Russell CT, Mewaldt RA, Luhmann JG Mason GM, von Rosenvinge TT, Cohen CMS Leske RA, Gomez-Herrero R, Klassen A, Galvin AB, Simunac KDC (2013b) The very unusual interplanetary coronal mass ejection of 2012 July 23: a blast wave mediated by solar energetic particles. Astrophys J 770:38-42

Ruzmaikin A, Feynman J (2014) The centennial Gleissberg cycle: origin and forcing of climate. ASPC 484:189

Savani NP, Shiota D, Kusano K, Vourlidas A, Lugaz N (2012) A study of the heliocentric dependence of shock standoff distance and geometry using $25 \mathrm{~d}$ magnetohydrodynamic simulations of coronal mass ejection driven shocks. Astrophys J 759:103

Scherliess L, Fejer BG (1997) Storm time dependence of equatorial disturbance dynamo zonal electric field. JGR 102:24037

Selhorst CL, Giménez de Castro CG, Válio A, Costa JER, Shibasaki K (2011) The behavior of the $17 \mathrm{GHz}$ solar radius and limb brightening in the spotless minimum XXIII/XXIV. Astrophys J 734:64-66

Selhorst CL, Costa JER, Giménez de Castro CG, Valio A, Pacini AA, Shibasaki K (2014) The $17 \mathrm{GHz}$ active region number. Astrophys J 790:134-139

Selwa M, Poedts S, DeVore CR (2013) Numerical simulations of dome-shaped EUV waves from different active-region configurations. SoPh 284:515-539

Shea MA, Smart DF (2008) Significant solar proton events for five solar cycles (1954-2007). In: Caballero R, D’Olivo JC, Medina-Tanco G, Nellen L, Sánchez FA, Valdés-Galicia JF (eds) Proceedings of the 30th international cosmic ray conference, vol 1. Universidad Nacional Autónoma de México, Mexico City, Mexico, p 261

Shea MA, Smart DF (2012) Space weather and the ground-level solar proton events of the 23rd solar cycle. Space Sci Rev 171:161-188

Sheeley NR, Hakala WN, Wang YM (2000) Detection of coronal mass ejection associated shock waves in the outer corona. JGRA 105:5081-5092

Shen C, Li G, Kong X, Hu J, Sun XD, Ding L, Chen Y, Wang Y, Xia L (2013a) Compound twin coronal mass ejections in the 2012 May 17 GLE event. Astrophys J 763:114

Shen CL, Wang YM, Gui B, Ye P, Wang S (2011) Kinematic evolution of a slow CME in corona viewed by STEREO-B on 8 October 2007. SoPh 269:389-400

Shen CL, Wang YM, Wang S, Liu Y, Vourlidas A, Miao B, Ye PZ, Liu JJ, Zhou ZJ (2012) Super-elastic collision of large-scale magnetized plasmoids in the heliosphere. Nat Phys 8:923-928

Shibasaki K (2013) Long-term global solar activity observed by the Nobeyama Radioheliograph. PASJ 65:17

Shibata K, Magara T (2011) Solar flares: magnetohydrodynamic processes. Living Rev Sol Phys 8:6

Shibata K, Isobe H, Hillier A, Choudhuri AR, Maehara H, Ishii TT, Shibayama T, Notsu S, Notsu Y, Nagao T, Honda S, Nogami D (2013) Can superflares occur on our Sun? Publ Astron Soc Jpn 65:49

Shimojo M (2013) Unusual migration of prominence activities in the southern hemisphere during cycles 23-24. PASJ 65:16

Siscoe G, Crooker NU, Clauer CR (2006) Dst of the Carrington storm of 1859 . AdSpR 38:173-179

Smith EJ, Tsurutani BT, Rosenberg RL (1978) Observations of the interplanetary sector structure up to heliographic latitudes of 16. JGR 83:A2717

Smith EJ, Wolf JH (1976) Observations of interaction regions and corotating shocks between one and five AU: Pioneers 10 and 11. GRL 3:137

Solomon SC, Qian L, Burns AG (2013) The anomalous ionosphere between solar cycles 23 and 24. JGR 118:6524-6535 
Sonett CP, Colburn DS, Davis L, Smith EJ, Coleman PJ (1964) Evidence for a collisionfree magneto-hydrodynamic shock in interplanetary space. PRL 13:153

Srivastava N, Joshi AD, Mathew SK (2014) On the onset of recurrent eruptions of a filament observed during August 2012. IAUS 300:495-496

Steinhilber F, Beer J (2013) Prediction of solar activity for the next 500 years. JGRA 118:1861-1867

Sterling AC, Moore RL, Harra LK (2011) Lateral offset of the coronal mass ejections from the $x$-flare of 2006 December 13 and its two precursor eruptions. Astrophys J 743:63-73

Sterling AC, Moore RL, Falconer DA, Knox JM (2014) New aspects of a lid-removal mechanism in the onset of an eruption sequence that produced a large solar energetic particle (SEP) event. Astrophys J 788:L20

Svalgaard L, Cliver EW, Kamide Y (2005) Sunspot cycle 24: smallest cycle in 100 years? GRL 32:L01104

Svalgaard L, Kamide Y (2013) Asymmetric solar polar field reversals. Astrophys J 763:23

Tan BL (2011) Multi-timescale solar cycles and their possible implications. Ap\&SS 332:65

Tan BL, Cheng Z (2013) The mid-term and long-term solar quasi-periodic cycles and the possible relationship with planetary motions. Ap\&SS 343:511

Temmer M, Rollett T, Mostl C, Veronig AM, Vrsnak B, Odstrcil D (2011) Influence of the ambient solar wind flow on the propagation behavior of interplanetary coronal mass ejections. Astrophys J 743:101

Temmer M, Vršnak B, Rollett T, Bein B, de Koning CA, Liu Y, Bosman E, Davies JA, Möstl C, Žic T, Veronig AM, Bothmer V, Harrison R, Nitta N, Bisi M, Flor O, Eastwood J, Odstrcil D, Forsyth R (2012) Characteristics of kinematics of a coronal mass ejection during the 2010 August 1 CME-CME interaction event. Astrophys J 749:57

Temmer M, Vrsnak B, Veronig AM (2013) The wave-driver system of the off-disk coronal wave of 17. SoPh 287:441-454

Temmer M, Veronig AM, Peinhart V, Vršnak B (2014) Asymmetry in the CME-CME interaction process for the events from 2011 February 14-15. Astrophys J 785:85

Thakur N, Gopalswamy N, Xie H, Mäkelä P, Yashiro S, Akiyama S, Davila JM (2014) Ground level enhancement in the 2014 January 6 solar energetic particle event. Astrophys J 790:L13

Thayer JP, Lei J, Forbes JM, Sutton EK, Nerem RS (2008) Thermospheric density oscillations due to periodic solar wind high-speed streams. JGR 113:A06307, doi:10.1029/2008JA013190

Thernisien A (2011) Implementation of the graduated cylindrical shell model for the three-dimensional reconstruction of coronal mass ejections. Astrophys JS 194:33-38

Thome GD, Wagner LS (1971) Electron density enhancements in the E and F regions of the ionosphere during solar flares. JGR 76:6883

Thomson NR, Rodger CJ, Dowden RL (2004) lonosphere gives the size of the greatest solar flare. GRL 31:L06803, doi:10.1029/2003GL019345

Thorne RM, Li W, Ni B, Ma Q, Bortnik J, Chen L, Baker DN, Spence HE, Reeves GD, Henderson MG, Kletzing CA, Kurth WS, Hospodarsky GB, Blake JB, Fennell JF, Claudepierre SG, Kanekal SG (2013) Rapid local acceleration of relativistic radiation-belt electrons by magnetospheric chorus. Nature 504:411

Tokumaru M, Fujiki K, Kojima M (2013) Evolution of global distribution of the solar wind from cycle 23 to the early phase of cycle 24. AIPC 1539:275-278

Tousey R (1973) The solar corona. In: Rycroft MJ, Runcorn SK (eds) Space research XIII. Akademie-Verlag, Berlin, p 713

Tsurutani BT, Smith EJ (1977) Two types of magnetospheric ELF chorus and their substorm dependences. JGR 82:5112

Tsurutani BT, Rodriguez P (1981). Upstream waves and particlesÖ An overview of Isee results, JGR, 86, A6, 4319.

Tsurutani BT, Smith EJ, Jones DE (1983) Waves observed upstream of interplanetary shocks. JGRA 88:5645

Tsurutani BT, Lin RP (1985) Acceleration of $>47 \mathrm{keV}$ ions and $>2 \mathrm{keV}$ electrons by interplanetary shocks at 1 AU. JGRA 90:1

Tsurutani BT, Gonzalez WD, Tang F, Akasofu SI, Smith EJ (1988) Origin of interplanetary southward magnetic fields responsible for major magnetic storms near solar maximum (1978-1979). JGR 91(A8):8519

Tsurutani BT, Gonzalez WD, Tang F, Lee YT, Okada M, Park D (1992a) Reply to L. J. Lanzerotti: solar wind ram pressure corrections and an estimation of the efficiency of viscous interaction. GRL 19:1993

Tsurutani BT, Gonzalez WD, Tang F, Lee YT (1992b) Great magnetic storms. GRL 19:73

Tsurutani BT, Gonzalez WD, Gonzalez ALC, Tang F, Arballo JK, Okada M (1995) Interplanetary origin of geomagnetic activity in the declining phase of the solar cycle. JGRA 100:21717
Tsurutani BT, Ho CM (1999) A review of discontinuities and Alfvén waves in interplanetary space: Ulysses results. Rev Geophys 37:517

Tsurutani BT, Wu ST, Zhang TX, Dryer M (2003a) Coronal mass ejection (CME)-induced shock formation, propagation and some temporally and spatially developing shock parameters relevant to particle energization. Astron Astrophys 412:293, doi:10.1051/0004-6361:20031413

Tsurutani BT, Gonzalez WD, Lakhina GS, Alex S (2003b) The extreme magnetic storm of 1-2 September 1859. JGRA 108:1268

Tsurutani BT, Mannucci A, lijima B, Abdu MA, Sobral JHA, Gonzalez W, Guarnieri F, Tsuda T, Saito A, Yumoto K, Fejer B, Fuller-Rowell TJ, Kozyra J, Foster JC, Coster A, Vasyliunas VM (2004) Global dayside ionospheric uplift and enhancement associated with interplanetary electric fields. JGR 109:A08302, doi:10,1029/2003JA010342

Tsurutani BT, Judge DL, Guarnieri FL, Gangopadhyay P, Jones AR, Nuttall J, Zambon GA, Didkovsky L, Mannucci AJ, lijima B, Meier RR, Immel TJ, Woods TN, Prasad S, Floyd L, Huba J, Solomon SC, Straus P, Viereck R (2005) The October 28, 2003 extreme EUV solar flare and resultant extreme ionospheric effects: comparison to other Halloween events and the Bastille day event. GRL 32:L03S09, doi:P10.1029/2004GL021475

Tsurutani BT, McPherron RL, Gonzalez WD, Lu G, Sobral JHA, Gopalswamy N (2006) Introduction to special section on corotating solar wind streams and recurrent geomagnetic activity. JGRA 111:7

Tsurutani BT, Echer E, Guarnieri FL, Kozyra JU (2008) CAWSES November 7-8, 2004 superstorm: complex solar and interplanetary features in the post-solar maximum phase. GRL 35:L06S05, doi:10.1029/2007GL031473

Tsurutani BT, Shibata K, Akasofu SI, Oka M (2009a) A two-step scenario for both solar flares and magnetospheric substorms: short duration energy storage. Earth, Planet and Space 61:1

Tsurutani BT, Verkhoglyadova OP, Mannucci AJ, Lakhina GS, Li G, Zank GP (2009b) A brief review of "solar flare effects" on the ionosphere. Radio Sci 44:RS0A17, doi:10.1029/2008RS004029

Tsurutani BT, Horne RB, Pickett JS, Santolik O, Schriver D, Verkhoglyadova OP (2010) Introduction to the special section on chorus: chorus and its role in space weather. JGR 115:A00F01, doi:10.1029/2010JA015870

Tsurutani BT, Echer E, Gonzalez WD (2011a) The solar and interplanetary causes of the recent minimum in the geomagnetic activity (MGA23): a combination of midlatitude small coronal holes, low IMF Bz variances, low solar wind speeds and low solar magnetic fields. Ann Geophys 29:1

Tsurutani BT, Lakhina GS, Verkhoglyadova OP, Gonzalez WD, Echer E, Guarnieri FL (2011b) A review of interplanetary discontinuities and their geomagnetic effects. J Atmos Sol-Terr Phy 73:5-19

Tsurutani, BT, Verkhoglzadova, OP, Mannucci, AJ, Lakhina, GS and Huba JD (2012). Extreme changes in the dayside ionosphere during a Carrington-type magnetic storm, J. Spa. Wea. Spa. Clim. doi:10.1051/swsc/2012004.

Tsurutani BT, Lakhina GS (2014) An extreme coronal mass ejection and consequences for the magnetosphere and Earth. GRL 41:287-292

Tsurutani BT, Echer E, Shibata K, Verkhoglyadova OP, Mannucci AJ, Gonzalez WD, Kozyra JU, Paetzold M (2014) The interplanetary causes of geomagnetic activity during the 7-17 March 2012 interval: a CAWSES II overview. J Spa Weath Spa Clim 4:A02, doi:10.1051/swsc/2013056

Usoskin IG (2013) A history of solar activity over millennia. LRSP 10:1

Vainio R, Ponni A, Battarbee M, Koskinen HEJ, Afanasiev A, Laitinen T (2014) A semi-analytical foreshock model for energetic storm particle events inside 1 AU. JSWSC 4:AA08

Vaisberg OL, Zastenker GN (1976) Solar wind and magnetosheath observations at earth during August 1972. SSRv 19:687-702

Vasyliunas VM (2011) The largest imaginable magnetic storm. JASTP 73:1444, doi:10.1016/jastp.2010.05.012

Veenadhari B, Selvakumaran R, Singh R, Maurya AK, Gopalswamy N, Kumar S, Kikuchi T (2012) Coronal mass ejection-driven shocks and the associated sudden commencements/sudden impulses. JGRA 117:4210

Verbanac G, Vršnak B, Veronig A, Temmer M (2011) Equatorial coronal holes, solar wind high-speed streams, and their geoeffectiveness. Astron Astrophys 526:A20

Verbanac G, Živković S, Vršnak B, Bandić M, Hojsak T (2013) Comparison of geoeffectiveness of coronal mass ejections and corotating interaction regions. Astron Astrophys 558:A85

Verkhoglyadova PO, Tsurutani BT, Mannucci AJ, Saito A, Araki T, Anderson D, Abdu M, Sobral JHA (2008) Simulation of PPEF effects in dayside low-latitude ionosphere for the October 30, 2003 superstorm. in Midlatitude lonospheric Dynamics and Disturbances, eds. P Kintner, A Coster, T Fuller-Rowell, A Mannucci, M Mendillo and R Heelis, 169, 181 
Verkhoglyadova OP, Tsurutani BT, Mannucci AJ, Mlynczak MG, Hunt LA, Komjathy A, Runge T (2011) lonospheric VTEC and thermospheric infrared emission dynamics during corotating interaction region and high-speed stream intervals at solar minimum: 25 March to 26 April 2008. JGR 116:A09325

Verkhoglyadova OP, Tsurutani BT, Mannucci AJ, Mlynczak MG, Hunt LA, Runge T (2013) Variability of ionospheric TEC during solar and geomagnetic minima (2008 and 2009): external high speed stream drivers. Ann Geophys 31:263

Verkhoglyadova OP, Tsurutani BT, Mannucci AJ, Mlynczak MG, Hunt LA, Paxton LJ (2014) lonospheric TEC, thermospheric cooling and $\Sigma[O / N 2]$ compositional changes during the 6-17 March 2012 magnetic storm interval (CAWSES II). JASTP 115:41

Veronig AM, Muhr N, Kienreich IW, Temmer M, Vrsnak B (2010) First observations of a dome-shaped large-scale coronal extreme-ultraviolet wave. Astrophys J 716:L57-L62

Vink J, Yamazaki R (2014) A critical shock mach number for particle acceleration in the absence of pre-existing cosmic rays: $M=\sqrt{5}$. Astrophys J 780:125

Vourlidas A, Wu ST, Wang AH, Subramanian P, Howard RA (2003) Direct detection of a coronal mass ejection-associated shock in large angle and spectrometric coronagraph experiment white-light images. Astrophys J 598:1392-1402

Vourlidas CA, Howard RA, Esfandiari E, Patsourakos S, Yashiro S, Michalek G (2011) Erratum: "comprehensive analysis of coronal mass ejection mass and energy properties over a full solar cycle" (2010, Astrophys J 722, 1522). Astrophys J 730:59

Vourlidas A, Lynch BJ, Howard RA, Li Y (2013) How many CMEs have flux ropes? Deciphering the signatures of shocks, flux ropes, and prominences in coronagraph observations of CMEs. SoPh 284:179-201

Vršnak B, Gopalswamy N (2002), Influence of the aerodynamic drag on the motion of interplanetary ejecta. JGR SSH 2-1, CitelD 1019, doi:10.1029/2001JA000120

Vršnak B, Zic T, Vrbanec D, Temmer M, Rollett T, Möstl C, et al (2013) Propagation of interplanetary coronal mass ejections: the drag-based model. SoPh 285:295-315

Vršnak B, Temmer M, Zic T, Taktakishvili A, Dumbovic M, Mostl C, Veronig AM, Mays ML, Odstrcil D (2014) Heliospheric propagation of coronal mass ejections: comparison of numerical WSA-ENLIL + cone model and analytical drag-based model. Astrophys JS 213:21

Wang X, Yan YH (2012) Analysis of the CME-driven shock from the SEP event that occurred on 2006 December 14. RAA 12:1535-1548

Wang Y-M, Colaninno R (2014) Is solar cycle 24 producing more coronal mass ejections than cycle 23? Astrophys J 784:LL27

Warmuth A, Mann G (2011) Kinematical evidence for physically different classes of large-scale coronal EUV waves. A\&A 532:AA151

Webb DF, Howard TA (2012) Coronal mass ejections: observations. LRSP 9:3

Wood BE, Wu C-C, Rouillard AP, Howard RA, Socker DG (2012) A coronal hole's effects on coronal mass ejection shock morphology in the inner heliosphere. Astrophys J 755:43

Wu CC, Lepping RP (2002) Effect of solar wind velocity on magnetic cloud-associated magnetic storm intensity. JGRA 107:A0236, doi:10.1029/2002JA009396

Xie H, St Cyr OC, Gopalswamy N, Odstrcil D, Cremades H (2013a) Understanding shock dynamics in the inner heliosphere with modeling and type II radio data: a statistical study. JGR 118(4):711-4723

Xie H, Gopalswamy N, St. Cyr OC (2013b) Near-sun flux-rope structure of CMEs. SoPh 284:47-58

Yakovchouk OS, Mursula K, Holappa L, Veselovsky IS, Karinen A (2012) Average properties of geomagnetic storms in 1932-2009. JGRA 117:3201

Yashiro S, Gopalswamy N, Akiyama S, Michalek G, Howard RA (2005) Visibility of coronal mass ejections as a function of flare location and intensity. JGRA 110:A12S05

Yashiro S, Gopalswamy N, Mäkelä P, Akiyama S (2013) Post-eruption arcades and interplanetary coronal mass ejections. SoPh 284:5-15

Yeh T (1995) A dynamical model of magnetic clouds. Astrophys J 438:975-984

Yermolaev YI, Lodkina IG, Nikolaeva NS, Yermolaev MY (2013) Occurrence rate of extreme magnetic storms. JGRA 118:4760-4765

Zhang J, Richardson IG, Webb DF, Gopalswamy N, Huttunen E, Kasper JC, Nitta NV, Poomvises W, Thompson BJ, Wu C-C Yashiro S, Zhukov AN (2007) Solar and interplanetary sources of major geomagnetic storms (Dst $<=-100 \mathrm{nT}$ ) during 1996-2005. JGR 112:10102

Zhang M, Flyer N, Low BC (2012a) Magnetic helicity of self-similar axisymmetric force-free fields. Astrophys J 755:78
Zhang J, Cheng X, Ding MD (2012b) The detection of an evolving magnetic flux rope in the suns corona. Nat Commun 3:747

Zigman V, Kudela K, Grubor D (2014) Response of the Earth's lower ionosphere to the ground level enhancement event of December 13, 2006. AdSR 53:763-775

Zolotova NV, Ponyavin DI (2013) Spatio-temporal sunspot impulses and reversal of the polar magnetic field on the sun. Ge \& Ae 53:945-948

Zolotova NV, Ponyavin DI (2014) Is the new grand minimum in progress? JGR 119:3281

\section{Submit your manuscript to a SpringerOpen ${ }^{\odot}$ journal and benefit from:}

- Convenient online submission

- Rigorous peer review

- Immediate publication on acceptance

- Open access: articles freely available online

- High visibility within the field

- Retaining the copyright to your article

Submit your next manuscript at $>$ springeropen.com 